\title{
Multi-Ion Bridged Pathway of N-Oxides to 1,3-Dipole Dilithium Oxide Complexes
}

Martin J. Neal, Sarah L. Hejnosz, Jeffrey J. Rohde ${ }^{1}$, Jeffrey D. Evanseck, and Thomas D. Montgomery*

Contribution from the Center for Computational Sciences and the Department of Chemistry and Biochemistry, Duquesne University, 600 Forbes Avenue, Pittsburgh, Pennsylvania 15282-1530.

${ }^{1}$ Department of Chemistry, Physics, and Engineering, Franciscan University of Steubenville, 1235 University Boulevard, Steubenville, OH 43952

* Corresponding author: montgomeryt1@duq.edu.

\section{Supporting Information}

\section{Content}

Confirmation of Activation Energy with Higher Order Theory............................................. 4

Structures for Confirmation of Activation Energy with Higher Order Thoery.........................5

$S 1_{\text {endo-Ts }}$ - First Deprotonation of Anti-planar Hydrogen with No THF ............................................ 5

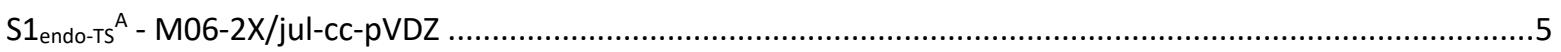

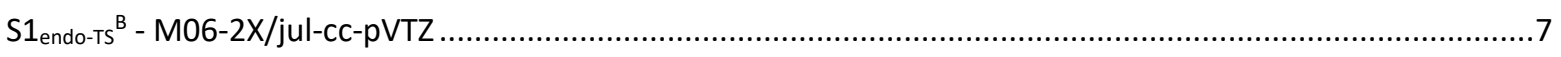

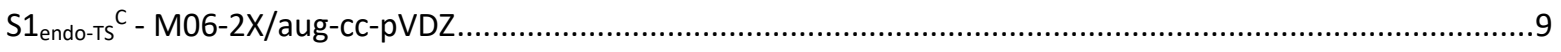

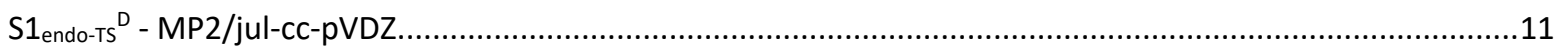

$S 1_{\text {exo-Ts }}$ - First Deprotonation of Syn-planar Hydrogen ................................................................. 12

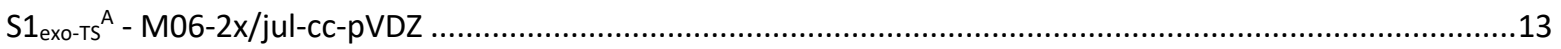

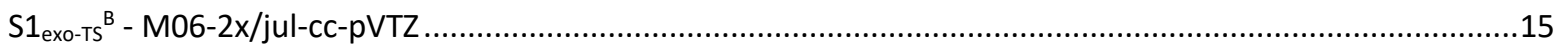

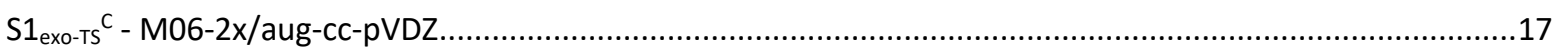

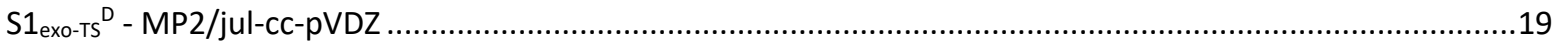

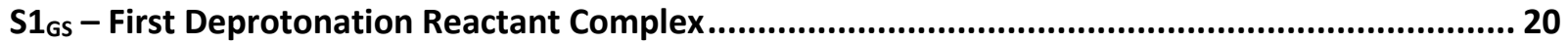

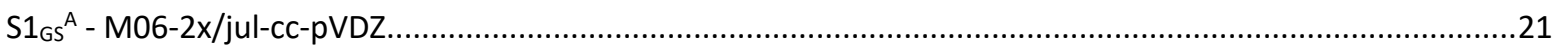

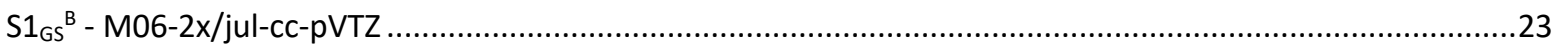

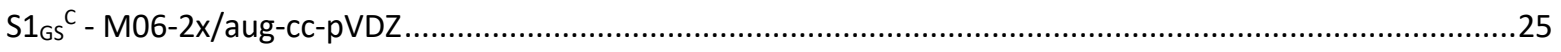

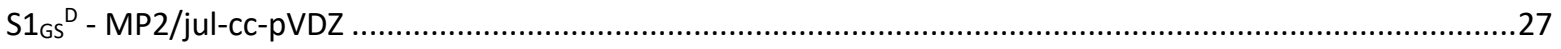

Expanded Figures for $\mathrm{N}$-Oxide to 1,3-dipole Mechanism................................................... 29

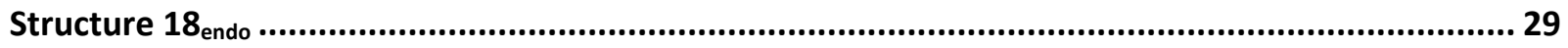

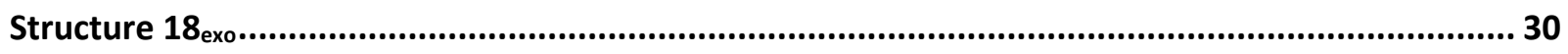

Structure $19 \mathrm{a}$

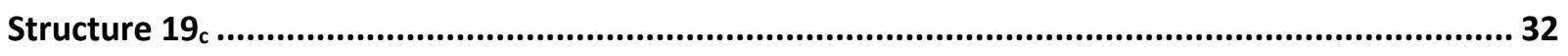




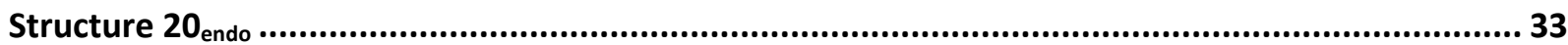

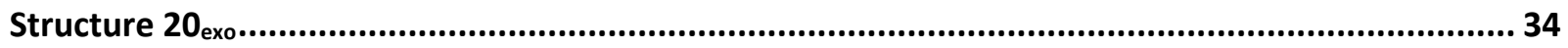

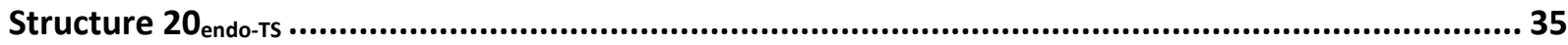

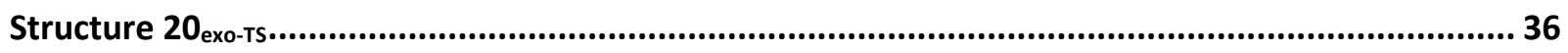

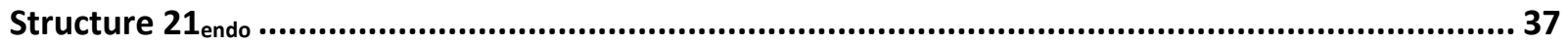

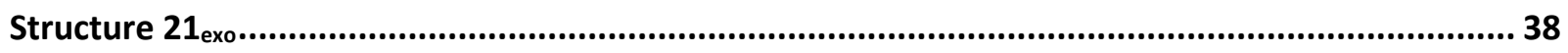

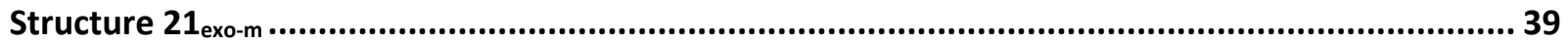

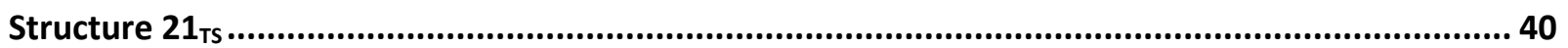

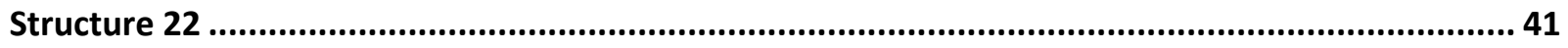

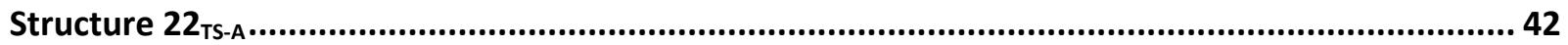

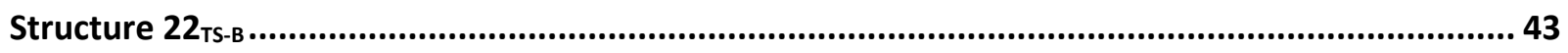

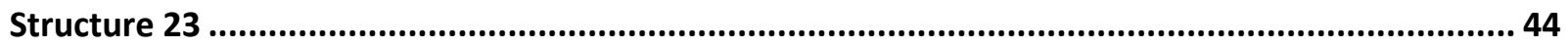

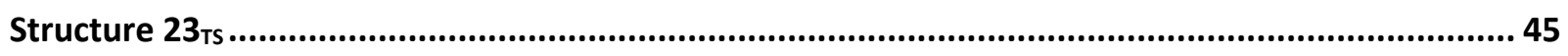

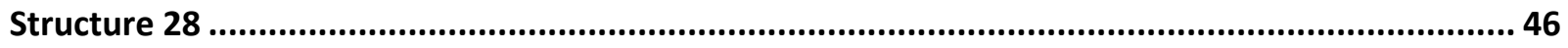

Gaussian Outputs for Structures of N-Oxide to 1,3-dipole Mechanism.............................. 47

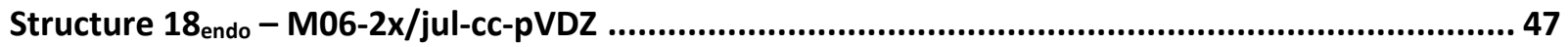

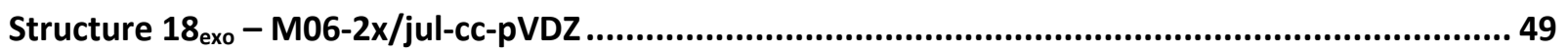

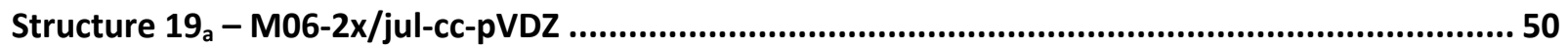

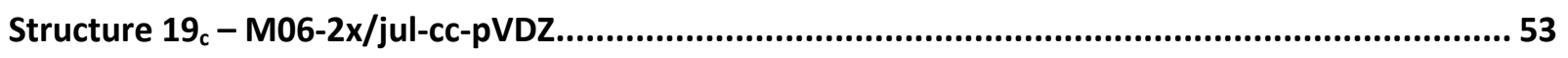

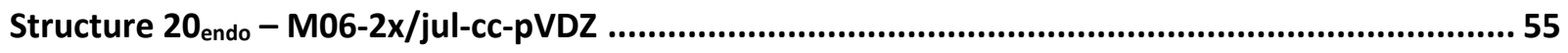

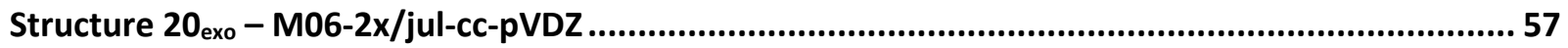

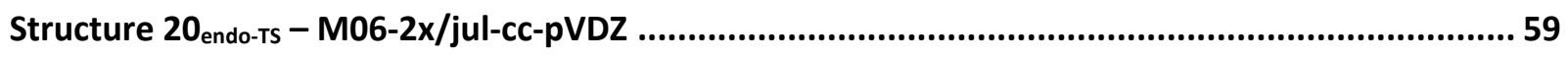

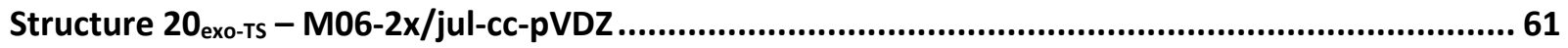

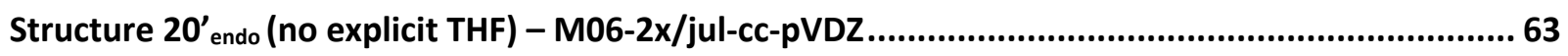

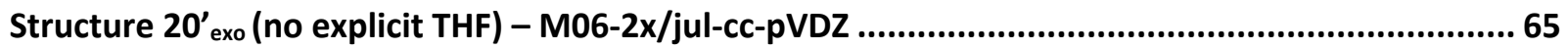

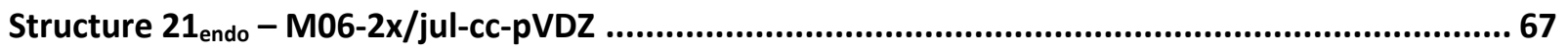

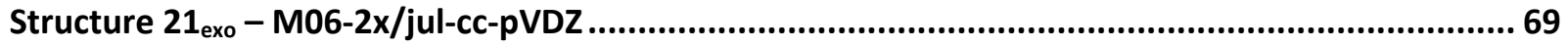

Structure $21_{\text {exo-m }}$ - M06-2x/jul-cc-pVDZ .......................................................................................... 71

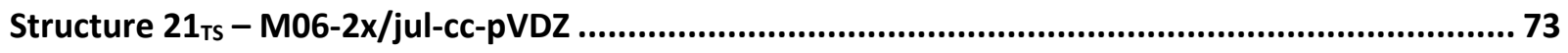




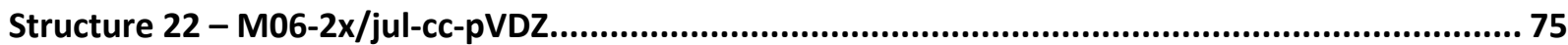

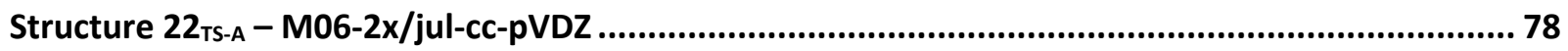

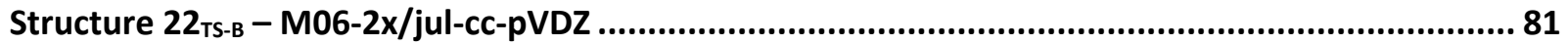

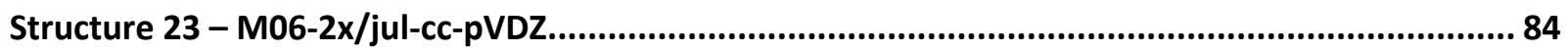

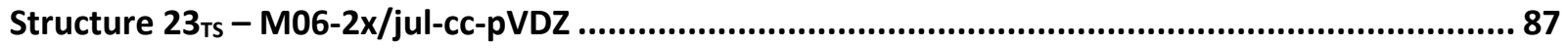

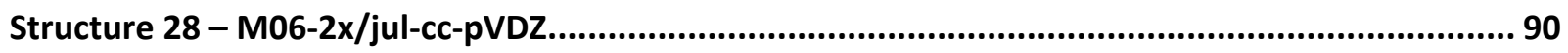

Models with Additional LDA Coordination ............................................................... 93

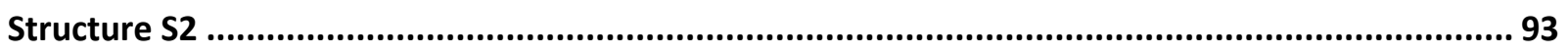

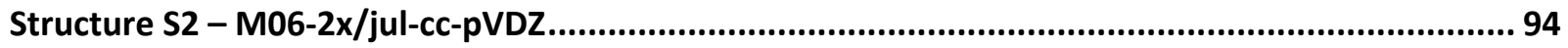

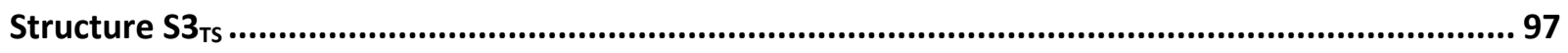

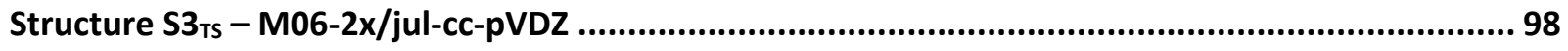

Energy Decomposition Analysis Calculations.......................................................... 101

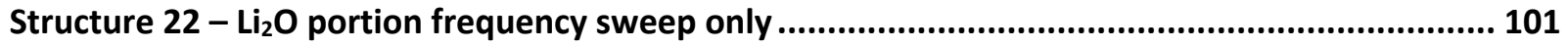

Structure 22 - Pyrrolidine portion frequency sweep only ........................................................... 103

Structure $22_{\mathrm{TS}-\mathrm{A}}-\mathrm{Li}_{2} \mathrm{O}$ portion frequency sweep only............................................................. 104

Structure $22_{\text {TS-A }}$ - Pyrrolidine portion frequency sweep only....................................................... 106

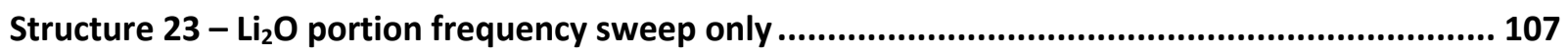

Structure 23 - Pyrrolidine portion frequency sweep only ......................................................... 109

Structure $23_{\mathrm{Ts}}-\mathrm{Li}_{2} \mathrm{O}$ portion frequency sweep only.............................................................. 110

Structure $23_{\mathrm{TS}}$ - Pyrrolidine portion frequency sweep only ....................................................... 112 


\section{Confirmation of Activation Energy with Higher Order Theory}

Calculations at higher levels of theory for the first deprotonation structures, both syn-planar $\left(\mathbf{T S}_{3}\right)$ and anti-planar $\left(\mathbf{T S}_{2}\right)$ orientation, were run to confirm the previous calculation preformed at M06-2X/jul-cc-pVDZ level of theory. DFT calculations were done at M06-2X/aug-cc-pVDZ and M06-2X/jul-cc-pVTZ. Additionally, second-order Møller-Plesset calculations were done at MP2/jul-cc-pVDZ level of theory. Across these different calculations there was not large deviations in the activation energies calculated with each transition structure. Utilizing MP2 lowered both activation enthalpies by approximately $1 \mathrm{kcal} / \mathrm{mol}$. When examining the difference in activation enthalpies between two orientations across the different levels of theory, the trend of approximately $7 \mathrm{kcal} / \mathrm{mol}$ difference holds. Therefore, calculations done at M06-2X/jul-cc-pVDZ level of theory represent good approximations for activation enthalpies and relative enthalpic differences.

TABLE S1. Activation enthalpies $(\Delta \mathrm{H})$ in $\mathrm{kcal} / \mathrm{mol}$ for anti-planar $\left(\mathbf{T S}_{\mathbf{2}}\right)$ and syn-planar $\left(\mathbf{T S}_{\mathbf{3}}\right)$ first deprotonation at different levels of theory, and the difference in activation enthalpies between the two orientations.

\begin{tabular}{|l|l|l|l|}
\hline Level of Theory & $\mathrm{S} 1_{\text {endo-TS }}$ & $\mathrm{S} 1_{\text {exo-TS }}$ & $\Delta \mathrm{E}_{\mathrm{a}}$ \\
\hline M06-2X/jul-cc-pVDZ & 20.0 & 12.5 & 7.5 \\
\hline M06-2X/jul-cc-pVTZ & 22.0 & 14.2 & 7.8 \\
\hline M06-2X/aug-cc-pVDZ & 20.2 & 12.7 & 7.5 \\
\hline MP2/jul-cc-pVDZ & 18.5 & 11.6 & 6.9 \\
\hline
\end{tabular}




\section{Structures for Confirmation of Activation Energy with Higher Order Thoery}

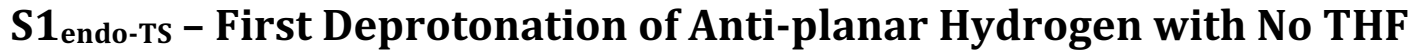

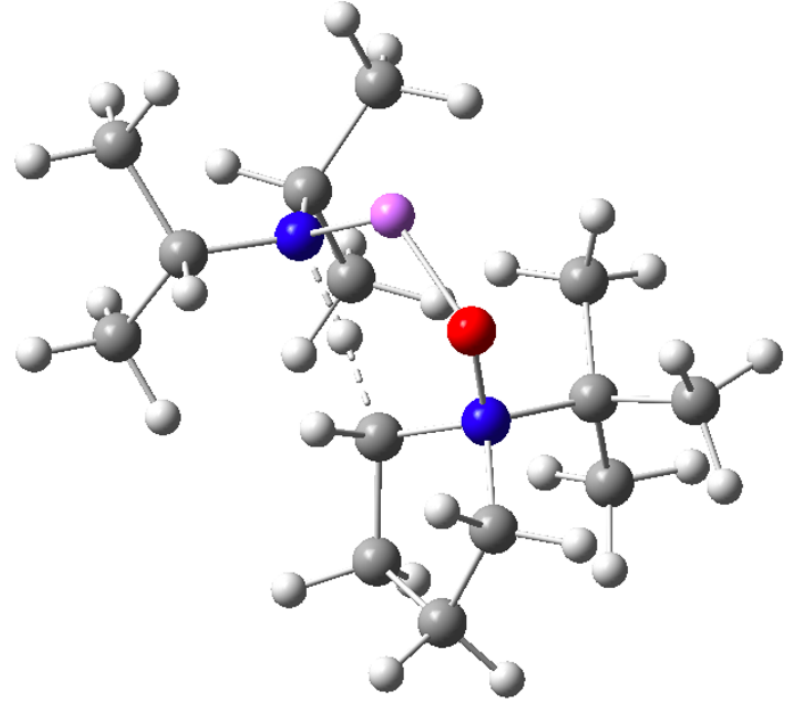

Figure S1. Representative Ball and Stick Model for $\mathbf{S} \mathbf{1}_{\text {endo-TS. }}$

S1 endo-Ts $^{\mathrm{A}}$ - M06-2X/jul-cc-pVDZ

Input:

$\#$ m062x/jul-cc-pvDz opt=(calcfc,noeigen,z-matrix,ts $)$ optcyc $=50$ freq $\mathrm{scrf}=(\mathrm{pcm}$, solvent $=\mathrm{THF})$

Optimzed Structure:

\begin{tabular}{cccccc}
$\begin{array}{c}\text { Center } \\
\text { Number }\end{array}$ & \multicolumn{2}{c}{$\begin{array}{c}\text { Atomic } \\
\text { Number }\end{array}$} & Atomic & \multicolumn{2}{c}{ Coordinates } \\
Type & X Angstroms) & Y & Z \\
\hline 1 & 7 & 0 & 0.000000 & 0.000000 & 0.000000 \\
2 & 8 & 0 & 0.000000 & 0.000000 & 1.389366 \\
3 & 6 & 0 & 1.454716 & 0.000000 & -0.501786 \\
4 & 6 & 0 & -0.742642 & -1.235207 & -0.432748 \\
5 & 6 & 0 & -0.835334 & 1.168753 & -0.499236 \\
6 & 6 & 0 & -2.216822 & -0.886130 & -0.247338 \\
7 & 6 & 0 & -2.261624 & 0.664606 & -0.243178 \\
8 & 1 & 0 & -0.400586 & -2.072801 & 0.179606 \\
9 & 1 & 0 & -0.505226 & -1.422413 & -1.485024 \\
10 & 1 & 0 & -2.596603 & -1.297610 & 0.694001 \\
11 & 1 & 0 & -2.810982 & -1.316610 & -1.061645 \\
12 & 1 & 0 & -2.649542 & 1.011461 & 0.722738
\end{tabular}




\begin{tabular}{rrrrrr}
13 & 1 & 0 & -2.938548 & 1.053746 & -1.014835 \\
14 & 1 & 0 & -0.560217 & 2.374019 & 0.323185 \\
15 & 1 & 0 & -0.678980 & 1.197710 & -1.586799 \\
16 & 6 & 0 & 2.089699 & -1.349468 & -0.166993 \\
17 & 6 & 0 & 1.537006 & 0.257535 & -2.008463 \\
18 & 6 & 0 & 2.204680 & 1.109333 & 0.229252 \\
19 & 1 & 0 & 1.739854 & -2.145736 & -0.835058 \\
20 & 1 & 0 & 3.176426 & -1.261267 & -0.289444 \\
21 & 1 & 0 & 1.875666 & -1.622511 & 0.871909 \\
22 & 1 & 0 & 0.871941 & -0.391380 & -2.589531 \\
23 & 1 & 0 & 1.315394 & 1.302023 & -2.251946 \\
24 & 1 & 0 & 2.565956 & 0.046293 & -2.326312 \\
25 & 1 & 0 & 1.636037 & 2.043714 & 0.199343 \\
26 & 1 & 0 & 2.407017 & 0.829205 & 1.268609 \\
27 & 1 & 0 & 3.166951 & 1.273959 & -0.271676 \\
28 & 3 & 0 & 0.268255 & 1.646482 & 2.168048 \\
29 & -1 & 0 & -0.104428 & 2.007525 & 0.707813 \\
30 & 7 & 0 & -0.400793 & 3.261789 & 1.234515 \\
31 & 6 & 0 & -1.743453 & 3.535994 & 1.764064 \\
32 & 6 & 0 & 0.280712 & 4.460691 & 0.733138 \\
33 & 1 & 0 & -2.147463 & 2.558931 & 2.081670 \\
34 & 6 & 0 & -1.729530 & 4.425178 & 3.012495 \\
35 & 6 & 0 & -2.718228 & 4.089821 & 0.714249 \\
36 & 1 & 0 & -0.337451 & 5.350255 & 0.938756 \\
37 & 6 & 0 & 1.615079 & 4.694394 & 1.447784 \\
38 & 6 & 0 & 0.479011 & 4.429758 & -0.789441 \\
39 & 1 & 0 & -2.744533 & 4.543468 & 3.418077 \\
40 & 1 & 0 & -1.093668 & 3.983866 & 3.791642 \\
41 & 1 & 0 & -1.342721 & 5.429048 & 2.786748 \\
42 & 1 & 0 & -3.733372 & 4.173508 & 1.127986 \\
43 & 1 & 0 & -2.411782 & 5.090701 & 0.377584 \\
44 & 1 & 0 & -2.750785 & 3.427600 & -0.160409 \\
45 & 1 & 0 & 2.110767 & 5.606371 & 1.083454 \\
46 & 1 & 0 & 1.450922 & 4.795687 & 2.529008 \\
47 & 1 & 0 & 2.299106 & 3.848906 & 1.282774 \\
48 & 1 & 0 & -0.484645 & 4.305733 & -1.300562 \\
49 & 1 & 0 & 0.943439 & 5.363397 & -1.137281 \\
50 & 1 & 0 & 1.126462 & 3.595863 & -1.094465 \\
------------------------------------------------------ \\
& & & & & \\
\hline 1 & & &
\end{tabular}

Thermochemistry:

Zero-point correction=

Thermal correction to Energy=

0.439390 (Hartree/Particle)

Thermal correction to Enthalpy=

0.461028

0.461972

Thermal correction to Gibbs Free Energy=

Sum of electronic and zero-point Energies=

0.391591

$-743.665256$

Sum of electronic and thermal Energies=

$-743.643618$

Sum of electronic and thermal Enthalpies=

$-743.642673$

Sum of electronic and thermal Free Energies=

$-743.713055$ 
S1 endo-Ts $^{B}$ - M06-2X/jul-cc-pVTZ

Input:

$\# \mathrm{m062x} / \mathrm{jul}-\mathrm{cc}-\mathrm{pvTz}$ opt=(calcfc,noeigen,z-matrix,ts $)$ optcyc $=50$ freq $\mathrm{scrf}=(\mathrm{pcm}, \mathrm{solvent}=\mathrm{THF})$

Optimzed Structure:

\begin{tabular}{|c|c|c|c|c|}
\hline \multirow{3}{*}{$\begin{array}{c}\text { Center } \\
\text { Number } \\
-1\end{array}$} & \multirow{2}{*}{$\begin{array}{l}\text { Atomic } \\
\text { Number }\end{array}$} & \multirow{2}{*}{$\begin{array}{l}\text { Atomic } \\
\text { Type }\end{array}$} & \multicolumn{2}{|c|}{ Coordinates (Angstroms } \\
\hline & & & $\mathrm{X}$ & $\mathrm{Z}$ \\
\hline & 7 & 000000 & 0.000000 & 0.000000 \\
\hline 2 & 8 & 0.000000 & 0.000000 & 1.389329 \\
\hline 3 & 6 & 1.452783 & 0.000000 & -0.503800 \\
\hline 4 & 6 & -0.743889 & -1.231540 & -0.434040 \\
\hline 5 & 6 & -0.836502 & 1.166976 & -0.496252 \\
\hline 6 & 6 & -2.211384 & -0.886032 & -0.229677 \\
\hline 7 & 6 & -2.261183 & 0.662203 & -0.250013 \\
\hline 8 & 1 & -0.397708 & -2.069274 & 0.162622 \\
\hline 9 & 1 & -0.519621 & -1.408405 & -1.483631 \\
\hline 10 & 1 & -2.565573 & -1.275765 & 0.722117 \\
\hline 11 & 1 & -2.816903 & -1.332304 & -1.016691 \\
\hline 12 & 1 & -2.656943 & 1.017516 & 0.700875 \\
\hline 13 & 1 & -2.927987 & 1.035350 & -1.027650 \\
\hline 14 & 1 & -0.572223 & 2.363016 & 0.341580 \\
\hline 15 & 1 & -0.672870 & 1.212321 & -1.573924 \\
\hline 16 & 6 & 2.087529 & -1.349297 & -0.179600 \\
\hline 17 & 6 & 1.533800 & 0.264465 & -2.006744 \\
\hline 18 & 6 & 2.208437 & 1.101346 & 0.227668 \\
\hline 19 & 1 & 1.728412 & -2.139719 & -0.837899 \\
\hline 20 & 1 & 3.165471 & -1.265027 & -0.316752 \\
\hline 21 & 1 & 1.889399 & -1.621950 & 0.855182 \\
\hline 22 & 1 & 0.869978 & -0.374932 & -2.586085 \\
\hline 23 & 1 & 1.315222 & 1.303850 & -2.243421 \\
\hline 24 & 1 & 2.554718 & 0.054767 & -2.326400 \\
\hline 25 & 1 & 1.650315 & 2.034418 & 0.197028 \\
\hline 26 & 1 & 2.406633 & 0.825655 & 1.261446 \\
\hline 27 & 1 & 3.166098 & 1.260597 & -0.268344 \\
\hline 28 & 3 & 0.258249 & 1.632884 & 2.177712 \\
\hline 29 & -1 & -0.118326 & 1.993648 & 0.725695 \\
\hline 30 & 7 & -0.417985 & 3.245733 & 1.261729 \\
\hline 31 & 6 & -1.755409 & 3.500650 & 1.808498 \\
\hline 32 & 6 & 0.248003 & 4.452334 & 0.764506 \\
\hline 33 & 1 & -2.145894 & 2.524654 & 2.119271 \\
\hline 34 & 6 & -1.737436 & 4.379552 & 3.061328 \\
\hline 35 & 6 & -2.744968 & 4.058218 & 0.778246 \\
\hline 36 & 1 & -0.367752 & 5.330783 & 0.983418 \\
\hline 37 & 6 & 1.588830 & 4.688052 & 1.461025 \\
\hline 38 & 6 & 0.431867 & 4.435322 & -0.757445 \\
\hline 39 & 1 & -2.740282 & 4.480790 & 3.480679 \\
\hline 40 & 1 & -1.086748 & 3.948133 & 3.823122 \\
\hline
\end{tabular}




$\begin{array}{rrrrrr}41 & 1 & 0 & -1.371934 & 5.383336 & 2.837002 \\ 42 & 1 & 0 & -3.749233 & 4.133351 & 1.199871 \\ 43 & 1 & 0 & -2.448207 & 5.056621 & 0.450120 \\ 44 & 1 & 0 & -2.783852 & 3.409456 & -0.097283 \\ 45 & 1 & 0 & 2.071391 & 5.600004 & 1.102136 \\ 46 & 1 & 0 & 1.442104 & 4.775168 & 2.538540 \\ 47 & 1 & 0 & 2.270191 & 3.854203 & 1.278336 \\ 48 & 1 & 0 & -0.529635 & 4.316753 & -1.258418 \\ 49 & 1 & 0 & 0.893327 & 5.362936 & -1.101336 \\ 50 & 1 & 0 & 1.069356 & 3.606528 & -1.070596\end{array}$

\section{Thermodynamics}

Zero-point correction=

Thermal correction to Energy=

Thermal correction to Enthalpy=

Thermal correction to Gibbs Free Energy=

Sum of electronic and zero-point Energies=

Sum of electronic and thermal Energies=

Sum of electronic and thermal Enthalpies=

Sum of electronic and thermal Free Energies=
0.440230 (Hartree/Particle)

0.461915

0.462859

0.392054

$-743.856323$

$-743.834639$

$-743.833694$

$-743.904499$ 


\section{S1 endo-TS $^{\mathrm{C}}$ - M06-2X/aug-cc-pVDZ}

Input:

$\#$ 062x/aug-cc-pvDz opt=(calcfc,noeigen,z-matrix,ts $)$ optcyc $=50$ freq scrf $=(\mathrm{pcm}$,solvent $=\mathrm{THF})$

Optimized Structure:

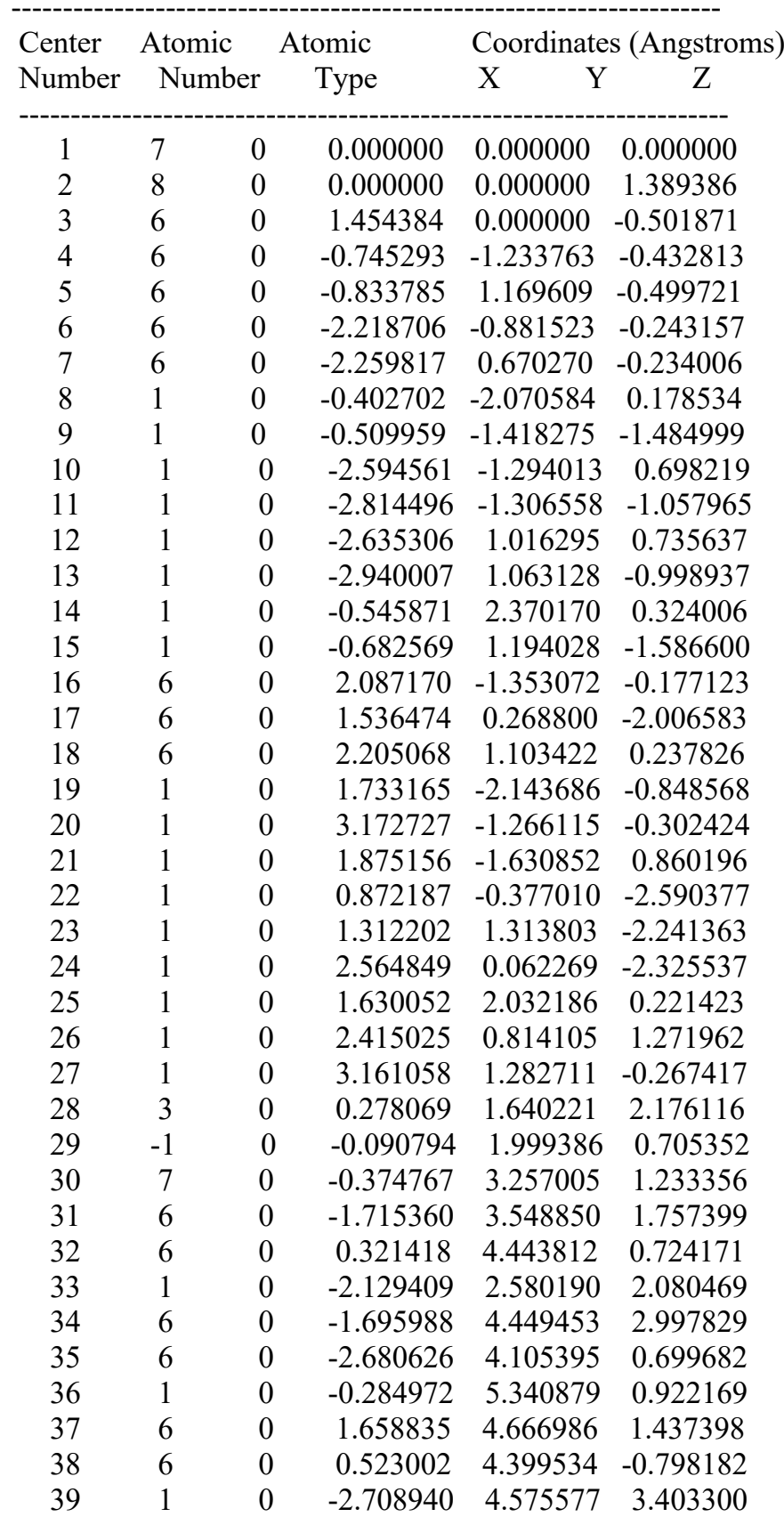




$\begin{array}{rrrrrr}40 & 1 & 0 & -1.060830 & 4.012671 & 3.778863 \\ 41 & 1 & 0 & -1.306955 & 5.448730 & 2.760647 \\ 42 & 1 & 0 & -3.696452 & 4.197951 & 1.106548 \\ 43 & 1 & 0 & -2.364328 & 5.101936 & 0.362587 \\ 44 & 1 & 0 & -2.711773 & 3.439084 & -0.170764 \\ 45 & 1 & 0 & 2.160616 & 5.573849 & 1.072021 \\ 46 & 1 & 0 & 1.496109 & 4.769587 & 2.517679 \\ 47 & 1 & 0 & 2.334682 & 3.816769 & 1.270248 \\ 48 & 1 & 0 & -0.438871 & 4.274758 & -1.310068 \\ 49 & 1 & 0 & 0.991717 & 5.327615 & -1.151000 \\ 50 & 1 & 0 & 1.169896 & 3.562550 & -1.091951\end{array}$

\section{Thermodynamics:}

Zero-point correction $=$ Thermal correction to Energy= Thermal correction to Enthalpy= Thermal correction to Gibbs Free Energy= Sum of electronic and zero-point Energies= Sum of electronic and thermal Energies= Sum of electronic and thermal Enthalpies= Sum of electronic and thermal Free Energies=
0.439284 (Hartree/Particle)

0.460851

0.461795

0.391751

$-743.678530$

$-743.656963$

$-743.656019$

$-743.726062$ 


\section{S1 endo-TS $^{\mathrm{D}}$ - MP2/jul-cc-pVDZ}

Input:

\#MP2/jul-cc-pvDz opt=(calcfc,noeigen,z-matrix,ts $)$ optcyc $=50$ freq scrf $=(\mathrm{pcm}$, solvent $=\mathrm{THF})$

Optimized Structure:

\begin{tabular}{|c|c|c|c|c|}
\hline \multirow{2}{*}{$\begin{array}{l}\text { Center } \\
\text { Number }\end{array}$} & Atomic & \multirow{2}{*}{$\begin{array}{r}\text { Atomic } \\
\text { Type }\end{array}$} & \multicolumn{2}{|c|}{ Coordinates (Angstroms } \\
\hline & Numbe & & $\mathrm{X}$ & $\mathrm{Z}$ \\
\hline 1 & 7 & 0.000000 & 0.000000 & 0.000000 \\
\hline 2 & 8 & 0.000000 & 0.000000 & 1.408445 \\
\hline 3 & 6 & 1.458155 & 0.000000 & -0.501871 \\
\hline 4 & 6 & -0.746101 & -1.247440 & -0.429525 \\
\hline 5 & 6 & -0.835151 & 1.174680 & -0.492137 \\
\hline 6 & 6 & -2.230111 & -0.883252 & -0.280044 \\
\hline 7 & 6 & -2.255120 & 0.672847 & -0.175360 \\
\hline 8 & 1 & -0.417029 & -2.076153 & 0.209773 \\
\hline 9 & 1 & -0.483553 & -1.451281 & -1.477714 \\
\hline 10 & 1 & -2.663132 & -1.356035 & 0.614157 \\
\hline 11 & 1 & -2.793728 & -1.242500 & -1.155190 \\
\hline 12 & 1 & -2.568393 & 0.957840 & 0.840789 \\
\hline 13 & 1 & -2.980221 & 1.118860 & -0.876405 \\
\hline 14 & 1 & -0.555045 & 2.442428 & 0.279993 \\
\hline 15 & 1 & -0.716350 & 1.178269 & -1.592986 \\
\hline 16 & 6 & 2.095236 & -1.356377 & -0.171061 \\
\hline 17 & 6 & 1.529591 & 0.261445 & -2.014503 \\
\hline 18 & 6 & 2.215492 & 1.109161 & 0.233710 \\
\hline 19 & 1 & 1.739220 & -2.157840 & -0.836620 \\
\hline 20 & 1 & 3.185696 & -1.267723 & -0.305366 \\
\hline 21 & 1 & 1.892079 & -1.627780 & 0.875031 \\
\hline 22 & 1 & 0.871885 & -0.403083 & -2.595135 \\
\hline 23 & 1 & 1.288308 & 1.305764 & -2.258821 \\
\hline 24 & 1 & 2.565842 & 0.066615 & -2.337149 \\
\hline 25 & 1 & 1.645357 & 2.046902 & 0.214012 \\
\hline 26 & 1 & 2.428418 & 0.824398 & 1.273946 \\
\hline 27 & 1 & 3.176745 & 1.276542 & -0.280114 \\
\hline 28 & 3 & 0.120466 & 1.716378 & 2.182952 \\
\hline 29 & -1 & -0.086584 & 2.100303 & 0.671778 \\
\hline 30 & 7 & -0.430472 & 3.370497 & 1.140620 \\
\hline 31 & 6 & -1.813156 & 3.709473 & 1.549691 \\
\hline 32 & 6 & 0.306220 & 4.538564 & 0.614753 \\
\hline 33 & 1 & -2.276155 & 2.756214 & 1.871776 \\
\hline 34 & 6 & -1.862004 & 4.654848 & 2.763118 \\
\hline 35 & 6 & -2.689038 & 4.256453 & 0.403456 \\
\hline 36 & 1 & -0.296980 & 5.454859 & 0.766694 \\
\hline 37 & 6 & 1.624538 & 4.760032 & 1.373931 \\
\hline 38 & 6 & 0.565993 & 4.440552 & -0.901870 \\
\hline 39 & 1 & -2.905059 & 4.828407 & 3.082010 \\
\hline 40 & 1 & -1.306631 & 4.223255 & 3.612166 \\
\hline
\end{tabular}




$\begin{array}{rrrrrr}41 & 1 & 0 & -1.418121 & 5.636900 & 2.528195 \\ 42 & 1 & 0 & -3.733277 & 4.386868 & 0.738720 \\ 43 & 1 & 0 & -2.326083 & 5.238388 & 0.055413 \\ 44 & 1 & 0 & -2.682151 & 3.561513 & -0.451003 \\ 45 & 1 & 0 & 2.164963 & 5.644526 & 0.990887 \\ 46 & 1 & 0 & 1.424508 & 4.912718 & 2.447101 \\ 47 & 1 & 0 & 2.288645 & 3.885553 & 1.268528 \\ 48 & 1 & 0 & -0.379666 & 4.317016 & -1.453107 \\ 49 & 1 & 0 & 1.067356 & 5.353943 & -1.268364 \\ 50 & 1 & 0 & 1.210517 & 3.580201 & -1.144241\end{array}$

Thermodynamics:

Zero-point correction= Thermal correction to Energy= Thermal correction to Enthalpy= Thermal correction to Gibbs Free Energy= Sum of electronic and zero-point Energies= Sum of electronic and thermal Energies= Sum of electronic and thermal Enthalpies= Sum of electronic and thermal Free Energies=

\author{
0.438611 (Hartree/Particle) \\ 0.460547 \\ 0.461492 \\ 0.390446 \\ $-741.669311$ \\ $-741.647375$ \\ $-741.646431$ \\ $-741.717476$
}

\title{
S1 $1_{\text {exo-TS }}$ - First Deprotonation of Syn-planar Hydrogen
}

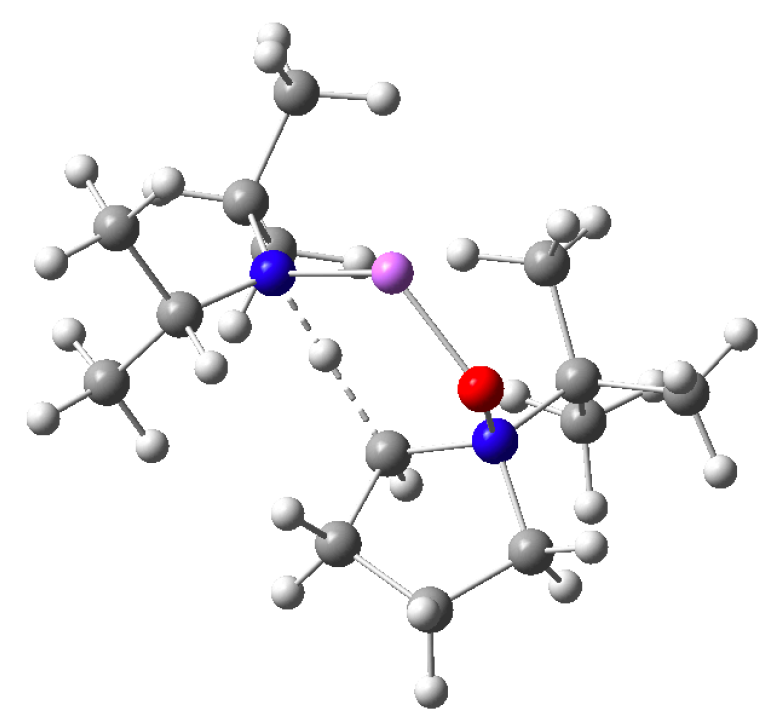

Figure S2. Representative Ball and Stick Model for $\mathbf{S} 1_{\text {exo-Ts. }}$ 


\section{S1 exo-TS $^{\mathrm{A}}$ - M06-2x/jul-cc-pVDZ}

Input:

$\#$ 062x/jul-cc-pvDz opt=(calcfc,noeigen,z-matrix,ts $)$ optcyc=50 freq $\mathrm{scrf}=(\mathrm{pcm}$, solvent $=\mathrm{THF})$

Optimized Structure:

\begin{tabular}{|c|c|c|c|c|c|}
\hline \multirow{2}{*}{$\begin{array}{l}\text { Center } \\
\text { Number }\end{array}$} & \multirow{2}{*}{\multicolumn{2}{|c|}{$\begin{array}{l}\text { Atomic } \\
\text { Number }\end{array}$}} & \multirow{2}{*}{$\begin{array}{l}\text { Atomic } \\
\text { Type }\end{array}$} & \multicolumn{2}{|c|}{ Coordinates (Angstroms } \\
\hline & & & & $\mathrm{X}$ & $\mathrm{Z}$ \\
\hline 1 & 7 & 0 & 0.000000 & 0.000000 & 0.000000 \\
\hline 2 & 8 & 0 & 0.000000 & 0.000000 & 1.385640 \\
\hline 3 & 6 & 0 & 1.480269 & 0.000000 & -0.455442 \\
\hline 4 & 6 & 0 & -0.763424 & -1.200243 & -0.518168 \\
\hline 5 & 6 & 0 & -0.836003 & 1.180916 & -0.494105 \\
\hline 6 & 6 & 0 & -1.426058 & -0.788103 & -1.838611 \\
\hline 7 & 6 & 0 & -1.350720 & 0.765869 & -1.869950 \\
\hline 8 & 1 & 0 & -1.502874 & -1.375801 & 0.266562 \\
\hline 9 & 1 & 0 & -0.117152 & -2.077002 & -0.588275 \\
\hline 10 & 1 & 0 & -2.464904 & -1.136552 & -1.848268 \\
\hline 11 & 1 & 0 & -0.929113 & -1.242370 & -2.703733 \\
\hline 12 & 1 & 0 & -2.344269 & 1.204198 & -2.026530 \\
\hline 13 & 1 & 0 & -0.726073 & 1.104513 & -2.704504 \\
\hline 14 & 1 & 0 & -1.705224 & 1.052378 & 0.175689 \\
\hline 15 & 1 & 0 & -0.417454 & 2.429221 & 0.063475 \\
\hline 16 & 6 & 0 & 2.129754 & -1.326818 & -0.055462 \\
\hline 17 & 6 & 0 & 1.619504 & 0.213426 & -1.960679 \\
\hline 18 & 6 & 0 & 2.196375 & 1.135258 & 0.270524 \\
\hline 19 & 1 & 0 & 1.904792 & -2.135522 & -0.760837 \\
\hline 20 & 1 & 0 & 3.218108 & -1.188001 & -0.053988 \\
\hline 21 & 1 & 0 & 1.813416 & -1.614587 & 0.952812 \\
\hline 22 & 1 & 0 & 1.052181 & -0.518629 & -2.544920 \\
\hline 23 & 1 & 0 & 1.321412 & 1.226759 & -2.249093 \\
\hline 24 & 1 & 0 & 2.679669 & 0.084853 & -2.213525 \\
\hline 25 & 1 & 0 & 1.622244 & 2.062532 & 0.208581 \\
\hline 26 & 1 & 0 & 2.382531 & 0.877861 & 1.318930 \\
\hline 27 & 1 & 0 & 3.166777 & 1.301335 & -0.214696 \\
\hline 28 & 3 & 0 & 0.116679 & 1.750577 & 2.042222 \\
\hline 29 & -1 & 0 & 0.084210 & 2.100000 & 0.423956 \\
\hline 30 & 7 & 0 & -0.298067 & 3.392664 & 0.966630 \\
\hline 31 & 6 & 0 & 0.507845 & 4.536866 & 0.531350 \\
\hline 32 & 6 & 0 & -1.695476 & 3.739835 & 1.247392 \\
\hline 33 & 1 & 0 & -0.067548 & 5.468740 & 0.664270 \\
\hline 34 & 6 & 0 & 1.772618 & 4.694809 & 1.380725 \\
\hline 35 & 6 & 0 & 0.865238 & 4.469046 & -0.962166 \\
\hline 36 & 1 & 0 & -2.198004 & 2.787125 & 1.493673 \\
\hline 37 & 6 & 0 & -1.861010 & 4.648391 & 2.470477 \\
\hline 38 & 6 & 0 & -2.448316 & 4.316887 & 0.037807 \\
\hline 39 & 1 & 0 & 2.374052 & 5.553708 & 1.048215 \\
\hline 40 & 1 & 0 & 1.503146 & 4.845904 & 2.434690 \\
\hline
\end{tabular}




$\begin{array}{rrrrrr}41 & 1 & 0 & 2.402043 & 3.795281 & 1.314802 \\ 42 & 1 & 0 & 1.388578 & 5.381923 & -1.281254 \\ 43 & 1 & 0 & 1.520964 & 3.613527 & -1.178355 \\ 44 & 1 & 0 & -0.043134 & 4.356587 & -1.568326 \\ 45 & 1 & 0 & -2.924310 & 4.827125 & 2.686230 \\ 46 & 1 & 0 & -1.399619 & 4.189528 & 3.355207 \\ 47 & 1 & 0 & -1.385585 & 5.626431 & 2.308465 \\ 48 & 1 & 0 & -2.371706 & 3.633510 & -0.818746 \\ 49 & 1 & 0 & -3.512056 & 4.463875 & 0.272784 \\ 50 & 1 & 0 & -2.034641 & 5.290478 & -0.261771\end{array}$

Thermodynamics:

Zero-point correction= Thermal correction to Energy= Thermal correction to Enthalpy= 0.440115 (Hartree/Particle) 0.461439 0.462383

Thermal correction to Gibbs Free Energy $=\quad 0.392620$

Sum of electronic and zero-point Energies $=\quad-743.653008$

Sum of electronic and thermal Energies $=\quad-743.631684$

Sum of electronic and thermal Enthalpies $=\quad-743.630740$

Sum of electronic and thermal Free Energies $=\quad-743.700503$ 


\section{$S 1_{\text {exo-TS }}{ }^{B}$ - M06-2x/jul-cc-pVTZ}

Input:

$\#$ 062x/jul-cc-pvTz opt=(calcfc,noeigen,z-matrix,ts $)$ optcyc $=50$ freq scrf $=(\mathrm{pcm}$, solvent $=\mathrm{THF})$

Optimized Structure:

\begin{tabular}{|c|c|c|c|c|}
\hline \multirow{2}{*}{$\begin{array}{l}\text { Center } \\
\text { Number }\end{array}$} & Atomic & \multirow{2}{*}{$\begin{array}{r}\text { Atomic } \\
\text { Type }\end{array}$} & \multicolumn{2}{|c|}{ Coordinates (Angstroms) } \\
\hline & Number & & $\mathrm{X}$ & Z \\
\hline 1 & 7 & 0.000000 & 0.000000 & 0.000000 \\
\hline 2 & 8 & 0.000000 & 0.000000 & 1.385818 \\
\hline 3 & 6 & 1.479219 & 0.000000 & -0.454202 \\
\hline 4 & 6 & -0.764290 & -1.196415 & -0.520759 \\
\hline 5 & 6 & -0.835741 & 1.179481 & -0.491612 \\
\hline 6 & 6 & -1.424967 & -0.781418 & -1.837376 \\
\hline 7 & 6 & -1.343653 & 0.770214 & -1.867482 \\
\hline 8 & 1 & -1.500636 & -1.372760 & 0.256403 \\
\hline 9 & 1 & -0.123362 & -2.068036 & -0.592947 \\
\hline 10 & 1 & -2.458463 & -1.122641 & -1.845472 \\
\hline 11 & 1 & -0.933972 & -1.232899 & -2.697806 \\
\hline 12 & 1 & -2.327203 & 1.210309 & -2.029875 \\
\hline 13 & 1 & -0.714665 & 1.102623 & -2.690985 \\
\hline 14 & 1 & -1.701280 & 1.053163 & 0.170424 \\
\hline 15 & 1 & -0.424932 & 2.425965 & 0.078641 \\
\hline 16 & 6 & 2.128152 & -1.325655 & -0.059567 \\
\hline 17 & 6 & 1.623061 & 0.217746 & -1.955426 \\
\hline 18 & 6 & 2.196561 & 1.130698 & 0.272179 \\
\hline 19 & 1 & 1.902903 & -2.127937 & -0.760741 \\
\hline 20 & 1 & 3.209385 & -1.188233 & -0.061395 \\
\hline 21 & 1 & 1.817377 & -1.614796 & 0.942228 \\
\hline 22 & 1 & 1.057151 & -0.504868 & -2.539804 \\
\hline 23 & 1 & 1.330847 & 1.226822 & -2.239196 \\
\hline 24 & 1 & 2.675967 & 0.087510 & -2.206189 \\
\hline 25 & 1 & 1.628187 & 2.053650 & 0.208339 \\
\hline 26 & 1 & 2.379244 & 0.879514 & 1.315262 \\
\hline 27 & 1 & 3.161439 & 1.296493 & -0.207940 \\
\hline 28 & 3 & 0.109221 & 1.740719 & 2.048041 \\
\hline 29 & -1 & 0.075138 & 2.095674 & 0.440356 \\
\hline 30 & 7 & -0.314909 & 3.383325 & 0.990630 \\
\hline 31 & 6 & 0.484424 & 4.533845 & 0.566989 \\
\hline 32 & 6 & -1.712857 & 3.717064 & 1.275478 \\
\hline 33 & 1 & -0.084678 & 5.457550 & 0.719037 \\
\hline 34 & 6 & 1.756507 & 4.678855 & 1.403209 \\
\hline 35 & 6 & 0.831733 & 4.489443 & -0.927084 \\
\hline 36 & 1 & -2.208448 & 2.766690 & 1.509169 \\
\hline 37 & 6 & -1.884999 & 4.611295 & 2.505120 \\
\hline 38 & 6 & -2.467527 & 4.306528 & 0.076241 \\
\hline 39 & 1 & 2.347915 & 5.540302 & 1.085046 \\
\hline 40 & 1 & 1.501822 & 4.804346 & 2.456545 \\
\hline
\end{tabular}




$\begin{array}{rrrrrr}41 & 1 & 0 & 2.383638 & 3.789606 & 1.310988 \\ 42 & 1 & 0 & 1.368552 & 5.390314 & -1.231459 \\ 43 & 1 & 0 & 1.464476 & 3.630280 & -1.158724 \\ 44 & 1 & 0 & -0.074648 & 4.404962 & -1.527578 \\ 45 & 1 & 0 & -2.941589 & 4.776271 & 2.725397 \\ 46 & 1 & 0 & -1.417575 & 4.155520 & 3.379009 \\ 47 & 1 & 0 & -1.424716 & 5.589010 & 2.349906 \\ 48 & 1 & 0 & -2.388682 & 3.640202 & -0.784314 \\ 49 & 1 & 0 & -3.524448 & 4.447953 & 0.309773 \\ 50 & 1 & 0 & -2.058058 & 5.278202 & -0.207615\end{array}$

Thermodynamics:

$\begin{array}{lc}\text { Zero-point correction= } & 0.441131 \text { (Hartree/Particle) } \\ \text { Thermal correction to Energy= } & 0.462362 \\ \text { Thermal correction to Enthalpy= } & 0.463307 \\ \text { Thermal correction to Gibbs Free Energy }= & 0.393808 \\ \text { Sum of electronic and zero-point Energies }= & -743.843513 \\ \text { Sum of electronic and thermal Energies }= & -743.822281 \\ \text { Sum of electronic and thermal Enthalpies }= & -743.821337 \\ \text { Sum of electronic and thermal Free Energies }= & -743.890836\end{array}$




\section{S1 exo-TS $^{\mathrm{C}}$ - M06-2x/aug-cc-pVDZ}

Input:

$\#$ m062x/aug-cc-pvDz opt=(calcfc,noeigen, $z$-matrix,ts $)$ optcyc $=50$ freq scrf $=(\mathrm{pcm}$, solvent $=\mathrm{THF})$

Optimized Structure

\begin{tabular}{|c|c|c|c|c|}
\hline \multirow{2}{*}{$\begin{array}{l}\text { Center } \\
\text { Number }\end{array}$} & Atomic & \multirow{2}{*}{$\begin{array}{r}\text { Atomic } \\
\text { Type }\end{array}$} & \multicolumn{2}{|c|}{ Coordinates (Angstroms } \\
\hline & Numbe & & $\mathrm{X}$ & $\mathrm{Z}$ \\
\hline 1 & 7 & 0.000000 & 0.000000 & 0.000000 \\
\hline 2 & 8 & 0.000000 & 0.000000 & 1.385658 \\
\hline 3 & 6 & 1.480174 & 0.000000 & -0.456806 \\
\hline 4 & 6 & -0.768850 & -1.197491 & -0.515594 \\
\hline 5 & 6 & -0.830902 & 1.182738 & -0.495955 \\
\hline 6 & 6 & -1.416516 & -0.787999 & -1.843885 \\
\hline 7 & 6 & -1.347443 & 0.767486 & -1.871819 \\
\hline 8 & 1 & -1.517475 & -1.356158 & 0.262226 \\
\hline 9 & 1 & -0.129983 & -2.079265 & -0.571469 \\
\hline 10 & 1 & -2.453054 & -1.140309 & -1.865729 \\
\hline 11 & 1 & -0.905302 & -1.238216 & -2.701463 \\
\hline 12 & 1 & -2.342102 & 1.201716 & -2.023487 \\
\hline 13 & 1 & -0.725265 & 1.108880 & -2.705040 \\
\hline 14 & 1 & -1.698751 & 1.063974 & 0.174246 \\
\hline 15 & 1 & -0.397533 & 2.427411 & 0.059305 \\
\hline 16 & 6 & 2.124433 & -1.337073 & -0.084473 \\
\hline 17 & 6 & 1.621013 & 0.245603 & -1.956986 \\
\hline 18 & 6 & 2.201011 & 1.118226 & 0.291818 \\
\hline 19 & 1 & 1.895052 & -2.129475 & -0.805491 \\
\hline 20 & 1 & 3.212502 & -1.202521 & -0.082119 \\
\hline 21 & 1 & 1.808315 & -1.643359 & 0.917471 \\
\hline 22 & 1 & 1.057728 & -0.476020 & -2.556080 \\
\hline 23 & 1 & 1.319384 & 1.263190 & -2.222645 \\
\hline 24 & 1 & 2.680931 & 0.125783 & -2.209927 \\
\hline 25 & 1 & 1.627165 & 2.044577 & 0.249485 \\
\hline 26 & 1 & 2.389259 & 0.840729 & 1.333414 \\
\hline 27 & 1 & 3.167592 & 1.298443 & -0.193116 \\
\hline 28 & 3 & 0.128399 & 1.750522 & 2.041549 \\
\hline 29 & -1 & 0.102362 & 2.093411 & 0.417842 \\
\hline 30 & 7 & -0.263040 & 3.393465 & 0.953681 \\
\hline 31 & 6 & 0.561562 & 4.516309 & 0.498918 \\
\hline 32 & 6 & -1.655303 & 3.766115 & 1.224920 \\
\hline 33 & 1 & 0.001034 & 5.457753 & 0.610634 \\
\hline 34 & 6 & 1.826432 & 4.673886 & 1.348947 \\
\hline 35 & 6 & 0.925271 & 4.411416 & -0.991575 \\
\hline 36 & 1 & -2.172341 & 2.826805 & 1.484348 \\
\hline 37 & 6 & -1.809662 & 4.698819 & 2.431243 \\
\hline 38 & 6 & -2.394115 & 4.335610 & 0.002531 \\
\hline 39 & 1 & 2.435487 & 5.521572 & 1.005361 \\
\hline 40 & 1 & 1.555553 & 4.841817 & 2.398937 \\
\hline
\end{tabular}




$\begin{array}{llllll}41 & 1 & 0 & 2.447087 & 3.768847 & 1.295375 \\ 42 & 1 & 0 & 1.450818 & 5.314450 & -1.329706 \\ 43 & 1 & 0 & 1.582470 & 3.551929 & -1.180143 \\ 44 & 1 & 0 & 0.020518 & 4.283906 & -1.598422 \\ 45 & 1 & 0 & -2.869628 & 4.892875 & 2.644544 \\ 46 & 1 & 0 & -1.351659 & 4.251161 & 3.322408 \\ 47 & 1 & 0 & -1.324692 & 5.667400 & 2.248672 \\ 48 & 1 & 0 & -2.321968 & 3.637458 & -0.841237 \\ 49 & 1 & 0 & -3.455707 & 4.500960 & 0.229004 \\ 50 & 1 & 0 & -1.964724 & 5.297952 & -0.307330\end{array}$

Thermodynamics:

Zero-point correction $=$

Thermal correction to Energy=

Thermal correction to Enthalpy=

Thermal correction to Gibbs Free Energy=

Sum of electronic and zero-point Energies=

Sum of electronic and thermal Energies=

Sum of electronic and thermal Enthalpies=

Sum of electronic and thermal Free Energies=
0.440251 (Hartree/Particle)
0.461401
0.462345

0.393265

$-743.666157$

$-743.645007$

$-743.644063$

$-743.713144$ 


\section{S1 exo-TS $^{D}$ - MP2/jul-cc-pVDZ}

Input:

\#MP2/jul-cc-pvDz opt=(calcfc,noeigen,z-matrix,ts $)$ optcyc $=50$ freq scrf $=(\mathrm{pcm}$, solvent=THF $)$

Optimized Structure:

\begin{tabular}{|c|c|c|c|c|}
\hline \multirow{2}{*}{$\begin{array}{l}\text { Center } \\
\text { Number }\end{array}$} & Atomic & \multirow{2}{*}{$\begin{array}{r}\text { Atomic } \\
\text { Type }\end{array}$} & \multicolumn{2}{|c|}{ Coordinates (Angstroms } \\
\hline & Numbe & & $\mathrm{X}$ & $\mathrm{Z}$ \\
\hline 1 & 7 & 0.000000 & 0.000000 & 0.000000 \\
\hline 2 & 8 & 0.000000 & 0.000000 & 1.405570 \\
\hline 3 & 6 & 1.482994 & 0.000000 & -0.455076 \\
\hline 4 & 6 & -0.771417 & -1.211314 & -0.510516 \\
\hline 5 & 6 & -0.831395 & 1.186449 & -0.498564 \\
\hline 6 & 6 & -1.413953 & -0.806342 & -1.848635 \\
\hline 7 & 6 & -1.353026 & 0.758533 & -1.877197 \\
\hline 8 & 1 & -1.523154 & -1.360529 & 0.275099 \\
\hline 9 & 1 & -0.126423 & -2.097126 & -0.555727 \\
\hline 10 & 1 & -2.453821 & -1.166985 & -1.878268 \\
\hline 11 & 1 & -0.891866 & -1.256298 & -2.707086 \\
\hline 12 & 1 & -2.356927 & 1.188504 & -2.030869 \\
\hline 13 & 1 & -0.732539 & 1.102835 & -2.718592 \\
\hline 14 & 1 & -1.701983 & 1.062838 & 0.181435 \\
\hline 15 & 1 & -0.403777 & 2.479534 & 0.030666 \\
\hline 16 & 6 & 2.137481 & -1.332747 & -0.062259 \\
\hline 17 & 6 & 1.622269 & 0.221793 & -1.966028 \\
\hline 18 & 6 & 2.196383 & 1.140086 & 0.277662 \\
\hline 19 & 1 & 1.895920 & -2.146467 & -0.763547 \\
\hline 20 & 1 & 3.230764 & -1.194144 & -0.084911 \\
\hline 21 & 1 & 1.842675 & -1.618675 & 0.957371 \\
\hline 22 & 1 & 1.070741 & -0.521932 & -2.558338 \\
\hline 23 & 1 & 1.308228 & 1.234538 & -2.254960 \\
\hline 24 & 1 & 2.691056 & 0.111771 & -2.214683 \\
\hline 25 & 1 & 1.609826 & 2.065172 & 0.224798 \\
\hline 26 & 1 & 2.390711 & 0.878730 & 1.327506 \\
\hline 27 & 1 & 3.165959 & 1.318771 & -0.216500 \\
\hline 28 & 3 & 0.029675 & 1.803041 & 2.072060 \\
\hline 29 & -1 & 0.104120 & 2.162973 & 0.393750 \\
\hline 30 & 7 & -0.318801 & 3.463129 & 0.879944 \\
\hline 31 & 6 & 0.519262 & 4.599447 & 0.448927 \\
\hline 32 & 6 & -1.739757 & 3.834794 & 1.055075 \\
\hline 33 & 1 & -0.053594 & 5.543320 & 0.545633 \\
\hline 34 & 6 & 1.756508 & 4.752294 & 1.348918 \\
\hline 35 & 6 & 0.943995 & 4.497245 & -1.030977 \\
\hline 36 & 1 & -2.269040 & 2.887764 & 1.286629 \\
\hline 37 & 6 & -1.970428 & 4.777068 & 2.249100 \\
\hline 38 & 6 & -2.407059 & 4.400269 & -0.216426 \\
\hline 39 & 1 & 2.385947 & 5.601562 & 1.027296 \\
\hline 40 & 1 & 1.448304 & 4.923580 & 2.393299 \\
\hline
\end{tabular}




$\begin{array}{rrrrrr}41 & 1 & 0 & 2.375924 & 3.839823 & 1.318341 \\ 42 & 1 & 0 & 1.520399 & 5.389548 & -1.334692 \\ 43 & 1 & 0 & 1.578285 & 3.611912 & -1.202472 \\ 44 & 1 & 0 & 0.063665 & 4.414560 & -1.687680 \\ 45 & 1 & 0 & -3.047150 & 4.979419 & 2.390016 \\ 46 & 1 & 0 & -1.576841 & 4.329190 & 3.176379 \\ 47 & 1 & 0 & -1.466391 & 5.746729 & 2.097467 \\ 48 & 1 & 0 & -2.285472 & 3.697887 & -1.056966 \\ 49 & 1 & 0 & -3.487085 & 4.560992 & -0.050549 \\ 50 & 1 & 0 & -1.966151 & 5.368975 & -0.506138\end{array}$

Thermodynamics:

Zero-point correction $=$

Thermal correction to Energy=

Thermal correction to Enthalpy=

0.438642 (Hartree/Particle)

0.460492

0.461436

Thermal correction to Gibbs Free Energy $=0.390427$

Sum of electronic and zero-point Energies $=\quad-741.658269$

Sum of electronic and thermal Energies $=\quad-741.636419$

Sum of electronic and thermal Enthalpies $=\quad-741.635474$

Sum of electronic and thermal Free Energies $=\quad-741.706484$

\section{S1 $1_{\mathrm{Gs}}$ - First Deprotonation Reactant Complex}

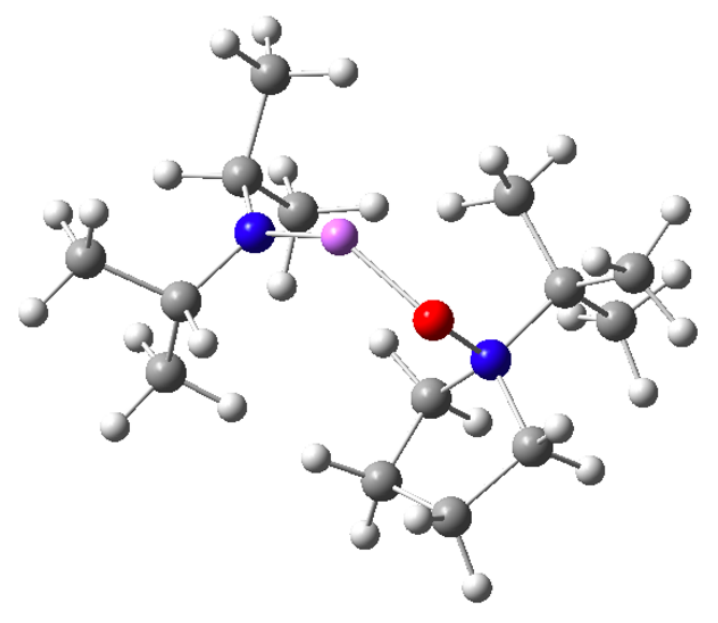

Figure S3. Representative Ball and Stick Model for $\mathbf{S} 1_{\mathbf{G S}}$ 


\section{$S 1_{G S}{ }^{A}-M 06-2 x / j u l-c c-p V D Z$}

Input:

$\#$ m062x/jul-cc-pvDz opt=(calcfc,noeigen,z-matrix $)$ freq scrf $=(\mathrm{pcm}$, solvent $=\mathrm{THF})$

Optimized Structures:

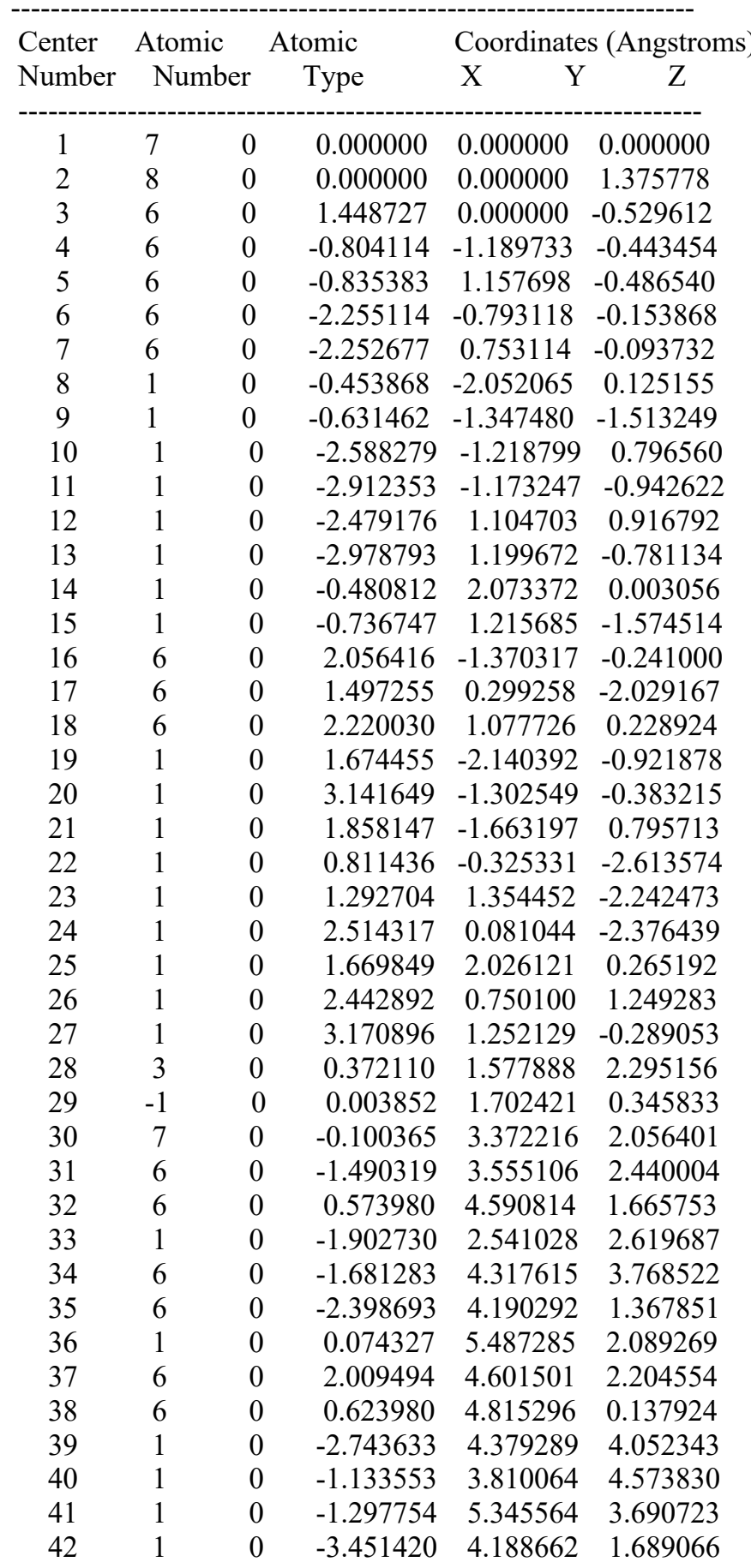

S21 


$\begin{array}{rrrrrr}43 & 1 & 0 & -2.108290 & 5.234941 & 1.180236 \\ 44 & 1 & 0 & -2.323438 & 3.640349 & 0.419348 \\ 45 & 1 & 0 & 2.574694 & 5.482899 & 1.864466 \\ 46 & 1 & 0 & 2.004266 & 4.586611 & 3.302887 \\ 47 & 1 & 0 & 2.539261 & 3.700958 & 1.854712 \\ 48 & 1 & 0 & -0.381168 & 4.781049 & -0.299143 \\ 49 & 1 & 0 & 1.079699 & 5.786098 & -0.116207 \\ 50 & 1 & 0 & 1.226032 & 4.026372 & -0.339864\end{array}$

Thermodynamics:

Zero-point correction=

Thermal correction to Energy=

Thermal correction to Enthalpy=

0.443714 (Hartree/Particle)

0.466010

0.466954

Thermal correction to Gibbs Free Energy= $\quad 0.393955$

Sum of electronic and zero-point Energies $=\quad-743.685850$

Sum of electronic and thermal Energies $=\quad-743.663555$

Sum of electronic and thermal Enthalpies $=\quad-743.662611$

Sum of electronic and thermal Free Energies $=\quad-743.735610$ 


\section{$S 1_{G S}{ }^{B}-M 06-2 x / j u l-c c-p V T Z$}

Input:

\#m062x/jul-cc-pvTz opt=(calcfc,noeigen,z-matrix $)$ freq scrf $=(\mathrm{pcm}$,solvent=THF $)$

Optimized Structure:

\begin{tabular}{|c|c|c|c|c|}
\hline \multirow{2}{*}{$\begin{array}{l}\text { Center } \\
\text { Number }\end{array}$} & Atomic & \multirow{2}{*}{$\begin{array}{r}\text { Atomic } \\
\text { Type }\end{array}$} & \multicolumn{2}{|c|}{ Coordinates (Angstroms } \\
\hline & Numbe & & $\mathrm{X}$ & $\mathrm{Z}$ \\
\hline 1 & 7 & 0.000000 & 0.000000 & 0.000000 \\
\hline 2 & 8 & 0.000000 & 0.000000 & 1.374732 \\
\hline 3 & 6 & 1.450915 & 0.000000 & -0.524147 \\
\hline 4 & 6 & -0.800426 & -1.190530 & -0.444537 \\
\hline 5 & 6 & -0.837759 & 1.150620 & -0.493507 \\
\hline 6 & 6 & -2.247254 & -0.800935 & -0.147615 \\
\hline 7 & 6 & -2.252888 & 0.743504 & -0.107288 \\
\hline 8 & 1 & -0.447712 & -2.048525 & 0.114890 \\
\hline 9 & 1 & -0.632892 & -1.343024 & -1.508547 \\
\hline 10 & 1 & -2.562464 & -1.210699 & 0.807419 \\
\hline 11 & 1 & -2.906926 & -1.194463 & -0.917243 \\
\hline 12 & 1 & -2.484337 & 1.103536 & 0.891482 \\
\hline 13 & 1 & -2.973469 & 1.176404 & -0.797226 \\
\hline 14 & 1 & -0.500063 & 2.065427 & -0.008324 \\
\hline 15 & 1 & -0.730973 & 1.208755 & -1.573481 \\
\hline 16 & 6 & 2.058597 & -1.365780 & -0.227261 \\
\hline 17 & 6 & 1.505049 & 0.287695 & -2.023161 \\
\hline 18 & 6 & 2.223073 & 1.079806 & 0.225310 \\
\hline 19 & 1 & 1.672745 & -2.137611 & -0.892464 \\
\hline 20 & 1 & 3.135508 & -1.301702 & -0.378321 \\
\hline 21 & 1 & 1.869486 & -1.647470 & 0.806754 \\
\hline 22 & 1 & 0.828148 & -0.339429 & -2.601552 \\
\hline 23 & 1 & 1.298067 & 1.333366 & -2.244660 \\
\hline 24 & 1 & 2.517624 & 0.073299 & -2.362988 \\
\hline 25 & 1 & 1.687969 & 2.029411 & 0.231378 \\
\hline 26 & 1 & 2.424534 & 0.782206 & 1.251639 \\
\hline 27 & 1 & 3.176030 & 1.232162 & -0.280985 \\
\hline 28 & 3 & 0.127929 & 1.563411 & 2.359691 \\
\hline 29 & -1 & -0.014588 & 1.701220 & 0.340485 \\
\hline 30 & 7 & -0.359843 & 3.351834 & 2.186668 \\
\hline 31 & 6 & -1.758429 & 3.509325 & 2.539202 \\
\hline 32 & 6 & 0.303453 & 4.579730 & 1.816139 \\
\hline 33 & 1 & -2.159263 & 2.496963 & 2.704747 \\
\hline 34 & 6 & -1.994205 & 4.263778 & 3.862926 \\
\hline 35 & 6 & -2.650091 & 4.137577 & 1.452566 \\
\hline 36 & 1 & -0.206068 & 5.461145 & 2.238701 \\
\hline 37 & 6 & 1.731854 & 4.598219 & 2.365772 \\
\hline 38 & 6 & 0.374042 & 4.819553 & 0.293652 \\
\hline 39 & 1 & -3.055060 & 4.300011 & 4.125224 \\
\hline 40 & 1 & -1.452536 & 3.774639 & 4.673418 \\
\hline
\end{tabular}




$\begin{array}{rrrrrr}41 & 1 & 0 & -1.637725 & 5.293630 & 3.793479 \\ 42 & 1 & 0 & -3.703474 & 4.119428 & 1.743360 \\ 43 & 1 & 0 & -2.371668 & 5.180208 & 1.281065 \\ 44 & 1 & 0 & -2.541593 & 3.599717 & 0.508938 \\ 45 & 1 & 0 & 2.288157 & 5.483344 & 2.046700 \\ 46 & 1 & 0 & 1.722314 & 4.562882 & 3.455987 \\ 47 & 1 & 0 & 2.268013 & 3.714909 & 2.005665 \\ 48 & 1 & 0 & -0.616494 & 4.783164 & -0.157341 \\ 49 & 1 & 0 & 0.827509 & 5.786360 & 0.054199 \\ 50 & 1 & 0 & 0.982801 & 4.041357 & -0.175296\end{array}$

Thermodynamics:

$\begin{array}{lc}\text { Zero-point correction= } & 0.445030 \text { (Hartree/Particle) } \\ \text { Thermal correction to Energy= } & 0.467122 \\ \text { Thermal correction to Enthalpy= } & 0.468066 \\ \text { Thermal correction to Gibbs Free Energy }= & 0.395529 \\ \text { Sum of electronic and zero-point Energies }= & -743.879374 \\ \text { Sum of electronic and thermal Energies }= & -743.857282 \\ \text { Sum of electronic and thermal Enthalpies }= & -743.856338 \\ \text { Sum of electronic and thermal Free Energies }= & -743.928876\end{array}$




\section{$S 1_{G S}{ }^{C}-M 06-2 x /$ aug-cc-pVDZ}

Input:

\#m062x/aug-cc-pvDz opt=(calcfc,noeigen,z-matrix $)$ freq $\mathrm{scrf}=(\mathrm{pcm}$,solvent=THF $)$

Optimized Structure:

\begin{tabular}{|c|c|c|c|c|}
\hline \multirow{2}{*}{$\begin{array}{l}\text { Center } \\
\text { Number }\end{array}$} & Atomic & \multirow{2}{*}{$\begin{array}{l}\text { Atomic } \\
\text { Type }\end{array}$} & \multicolumn{2}{|c|}{ Coordinates (Angstroms) } \\
\hline & Number & & $\mathrm{X}$ & Z \\
\hline 1 & 7 & 0.000000 & 0.000000 & 0.000000 \\
\hline 2 & 8 & 0.000000 & 0.000000 & 1.374533 \\
\hline 3 & 6 & 1.479518 & 0.000000 & -0.482917 \\
\hline 4 & 6 & -0.816697 & -1.178800 & -0.477935 \\
\hline 5 & 6 & -0.794928 & 1.173330 & -0.537026 \\
\hline 6 & 6 & -1.414797 & -0.813671 & -1.833598 \\
\hline 7 & 6 & -1.425746 & 0.735556 & -1.860092 \\
\hline 8 & 1 & -1.593068 & -1.254746 & 0.286201 \\
\hline 9 & 1 & -0.212747 & -2.084260 & -0.454353 \\
\hline 10 & 1 & -2.424609 & -1.226322 & -1.911074 \\
\hline 11 & 1 & -0.832732 & -1.225451 & -2.662268 \\
\hline 12 & 1 & -2.444648 & 1.128115 & -1.921900 \\
\hline 13 & 1 & -0.873864 & 1.118150 & -2.721657 \\
\hline 14 & 1 & -1.553177 & 1.332452 & 0.232676 \\
\hline 15 & 1 & -0.165966 & 2.063974 & -0.569657 \\
\hline 16 & 6 & 2.092432 & -1.367507 & -0.190653 \\
\hline 17 & 6 & 1.599218 & 0.322318 & -1.971777 \\
\hline 18 & 6 & 2.221563 & 1.065236 & 0.326725 \\
\hline 19 & 1 & 1.805525 & -2.121815 & -0.931786 \\
\hline 20 & 1 & 3.182675 & -1.264270 & -0.230470 \\
\hline 21 & 1 & 1.811410 & -1.703121 & 0.812762 \\
\hline 22 & 1 & 1.033488 & -0.368766 & -2.602255 \\
\hline 23 & 1 & 1.305018 & 1.353354 & -2.194854 \\
\hline 24 & 1 & 2.656051 & 0.215718 & -2.240223 \\
\hline 25 & 1 & 1.656933 & 2.000842 & 0.397791 \\
\hline 26 & 1 & 2.438023 & 0.703770 & 1.336029 \\
\hline 27 & 1 & 3.173189 & 1.277175 & -0.173423 \\
\hline 28 & 3 & 0.239845 & 1.631609 & 2.249761 \\
\hline 29 & -1 & 0.370542 & 1.694486 & -0.313460 \\
\hline 30 & 7 & -0.114483 & 3.425405 & 1.847568 \\
\hline 31 & 6 & 0.658496 & 4.598728 & 1.510220 \\
\hline 32 & 6 & -1.542861 & 3.658192 & 1.967153 \\
\hline 33 & 1 & 0.153309 & 5.531042 & 1.836974 \\
\hline 34 & 6 & 2.011360 & 4.562378 & 2.230631 \\
\hline 35 & 6 & 0.925874 & 4.769006 & -0.004711 \\
\hline 36 & 1 & -2.012378 & 2.662598 & 2.096835 \\
\hline 37 & 6 & -1.943196 & 4.470332 & 3.218476 \\
\hline 38 & 6 & -2.230621 & 4.286192 & 0.736374 \\
\hline 39 & 1 & 2.655515 & 5.407316 & 1.947228 \\
\hline 40 & 1 & 1.864582 & 4.575464 & 3.317791 \\
\hline
\end{tabular}




$\begin{array}{rrrrrr}41 & 1 & 0 & 2.537342 & 3.631232 & 1.968976 \\ 42 & 1 & 0 & 1.436646 & 5.720083 & -0.220820 \\ 43 & 1 & 0 & 1.572012 & 3.955336 & -0.366673 \\ 44 & 1 & 0 & -0.006392 & 4.741598 & -0.577927 \\ 45 & 1 & 0 & -3.035097 & 4.575658 & 3.305337 \\ 46 & 1 & 0 & -1.566002 & 3.974796 & 4.121903 \\ 47 & 1 & 0 & -1.513042 & 5.481070 & 3.179183 \\ 48 & 1 & 0 & -2.009126 & 3.710115 & -0.171635 \\ 49 & 1 & 0 & -3.321152 & 4.319957 & 0.869685 \\ 50 & 1 & 0 & -1.881489 & 5.316573 & 0.580402\end{array}$

\section{Thermodynamics:}

Zero-point correction $=$

Thermal correction to Energy=

Thermal correction to Enthalpy=

Thermal correction to Gibbs Free Energy=

Sum of electronic and zero-point Energies=

Sum of electronic and thermal Energies=

Sum of electronic and thermal Enthalpies=

Sum of electronic and thermal Free Energies=

\author{
0.444752 (Hartree/Particle) \\ 0.466451 \\ 0.467395 \\ 0.396189 \\ $-743.689849$ \\ $-743.668151$ \\ $-743.667207$ \\ $-743.738412$
}




\section{$S 1_{G S}{ }^{D}$ - MP2/jul-cc-pVDZ}

Input:

\#MP2/jul-cc-pvDz opt $=($ calcfc,noeigen, $z$-matrix $)$ optcyc $=50$ freq scrf $=(\mathrm{pcm}$, solvent $=\mathrm{THF})$

Optimized Structure:

\begin{tabular}{|c|c|c|c|c|}
\hline \multirow{2}{*}{$\begin{array}{l}\text { Center } \\
\text { Number }\end{array}$} & Atomic & \multirow{2}{*}{$\begin{array}{r}\text { Atomic } \\
\text { Type }\end{array}$} & \multicolumn{2}{|c|}{ Coordinates (Angstroms } \\
\hline & Numbe & & $\mathrm{X}$ & $\mathrm{Z}$ \\
\hline 1 & 7 & 0.000000 & 0.000000 & 0.000000 \\
\hline 2 & 8 & 0.000000 & 0.000000 & 1.391882 \\
\hline 3 & 6 & 1.453212 & 0.000000 & -0.530445 \\
\hline 4 & 6 & -0.800652 & -1.203248 & -0.442180 \\
\hline 5 & 6 & -0.844748 & 1.158469 & -0.489606 \\
\hline 6 & 6 & -2.256079 & -0.814757 & -0.136271 \\
\hline 7 & 6 & -2.268110 & 0.739873 & -0.103051 \\
\hline 8 & 1 & -0.435241 & -2.066914 & 0.123491 \\
\hline 9 & 1 & -0.635592 & -1.352002 & -1.519376 \\
\hline 10 & 1 & -2.571428 & -1.228507 & 0.830393 \\
\hline 11 & 1 & -2.922961 & -1.216930 & -0.913021 \\
\hline 12 & 1 & -2.515328 & 1.108716 & 0.900529 \\
\hline 13 & 1 & -2.992831 & 1.167789 & -0.811712 \\
\hline 14 & 1 & -0.505972 & 2.080035 & 0.010865 \\
\hline 15 & 1 & -0.735041 & 1.221403 & -1.581444 \\
\hline 16 & 6 & 2.075014 & -1.363920 & -0.210518 \\
\hline 17 & 6 & 1.495928 & 0.262644 & -2.043922 \\
\hline 18 & 6 & 2.223406 & 1.106384 & 0.198846 \\
\hline 19 & 1 & 1.692816 & -2.157863 & -0.870457 \\
\hline 20 & 1 & 3.162827 & -1.290297 & -0.367824 \\
\hline 21 & 1 & 1.891066 & -1.633402 & 0.839284 \\
\hline 22 & 1 & 0.821947 & -0.394078 & -2.614738 \\
\hline 23 & 1 & 1.271498 & 1.311423 & -2.288071 \\
\hline 24 & 1 & 2.522565 & 0.054132 & -2.385030 \\
\hline 25 & 1 & 1.686675 & 2.066267 & 0.170320 \\
\hline 26 & 1 & 2.420654 & 0.833555 & 1.244663 \\
\hline 27 & 1 & 3.190528 & 1.241028 & -0.312300 \\
\hline 28 & 3 & 0.102375 & 1.651326 & 2.344835 \\
\hline 29 & -1 & -0.020825 & 1.713401 & 0.357582 \\
\hline 30 & 7 & -0.395841 & 3.468324 & 2.030177 \\
\hline 31 & 6 & -1.809675 & 3.668328 & 2.359324 \\
\hline 32 & 6 & 0.285623 & 4.689787 & 1.622609 \\
\hline 33 & 1 & -2.230627 & 2.658121 & 2.566489 \\
\hline 34 & 6 & -2.048084 & 4.489669 & 3.652811 \\
\hline 35 & 6 & -2.682386 & 4.267061 & 1.227786 \\
\hline 36 & 1 & -0.234808 & 5.599523 & 2.003655 \\
\hline 37 & 6 & 1.710496 & 4.722755 & 2.205140 \\
\hline 38 & 6 & 0.389281 & 4.864643 & 0.084628 \\
\hline 39 & 1 & -3.125372 & 4.571869 & 3.891413 \\
\hline 40 & 1 & -1.537533 & 4.008589 & 4.503186 \\
\hline
\end{tabular}




$\begin{array}{rrrrrr}41 & 1 & 0 & -1.650292 & 5.514035 & 3.549776 \\ 42 & 1 & 0 & -3.750833 & 4.275969 & 1.512885 \\ 43 & 1 & 0 & -2.386268 & 5.308544 & 1.012857 \\ 44 & 1 & 0 & -2.575116 & 3.680419 & 0.300490 \\ 45 & 1 & 0 & 2.289821 & 5.592424 & 1.843157 \\ 46 & 1 & 0 & 1.676461 & 4.754957 & 3.306558 \\ 47 & 1 & 0 & 2.248954 & 3.805209 & 1.905954 \\ 48 & 1 & 0 & -0.601685 & 4.814563 & -0.391158 \\ 49 & 1 & 0 & 0.854584 & 5.832602 & -0.185925 \\ 50 & 1 & 0 & 1.012096 & 4.059236 & -0.344098\end{array}$

Thermodynamics:

$\begin{array}{lc}\text { Zero-point correction= } & 0.442983 \text { (Hartree/Particle) } \\ \text { Thermal correction to Energy= } & 0.465620 \\ \text { Thermal correction to Enthalpy= } & 0.466564 \\ \text { Thermal correction to Gibbs Free Energy }= & 0.392645 \\ \text { Sum of electronic and zero-point Energies }= & -741.688535 \\ \text { Sum of electronic and thermal Energies }= & -741.665897 \\ \text { Sum of electronic and thermal Enthalpies }= & -741.664953 \\ \text { Sum of electronic and thermal Free Energies }= & -741.738873\end{array}$




\section{Expanded Figures for N-Oxide to 1,3-dipole Mechanism}

\section{Structure 18 endo}

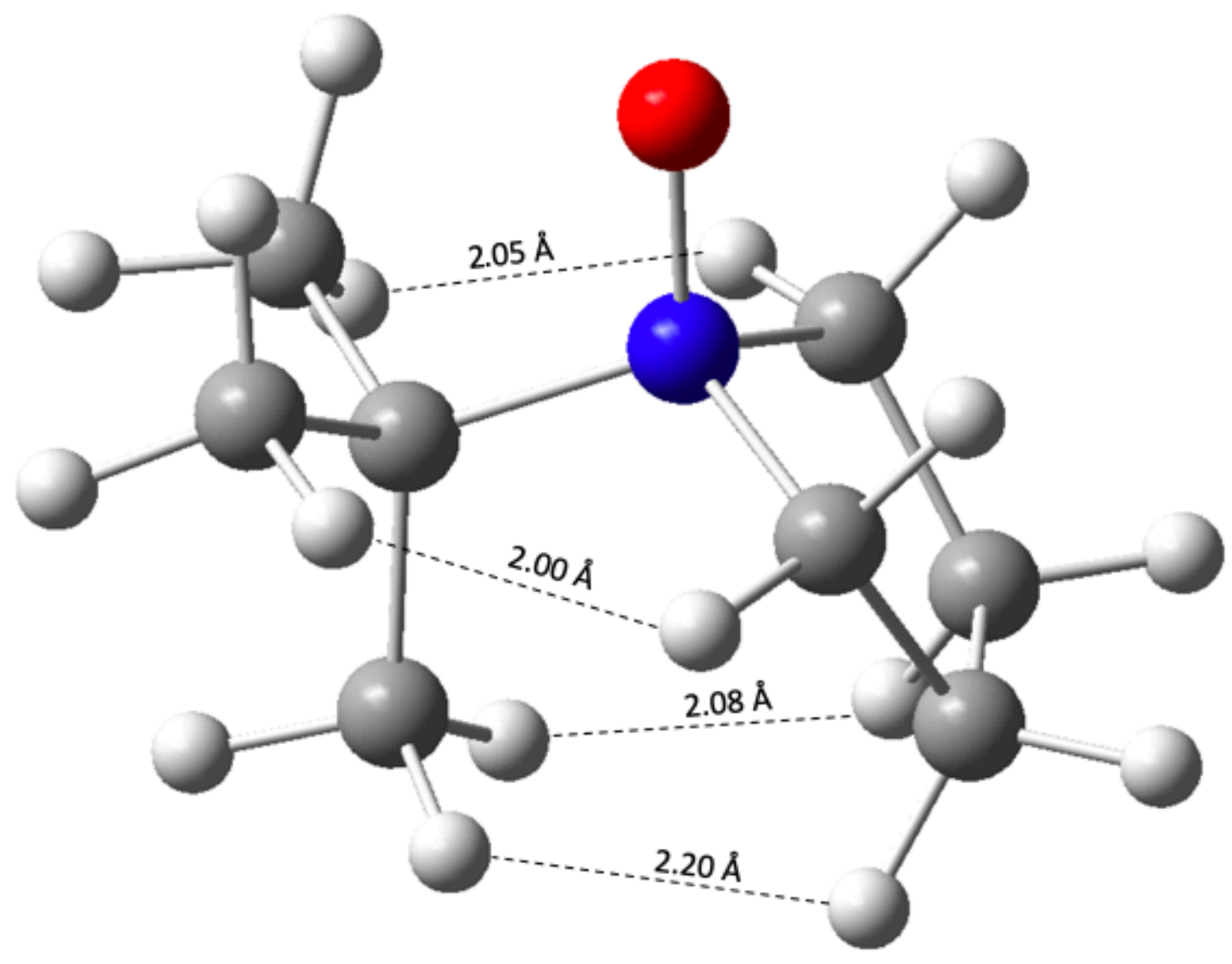

Figure S4. Ball and Stick Model for $18_{\text {endo }}$ with key atom distances indicated. Color denotes chemical composition; grey=car- bon, white=hydrogen, blue=nitrogen, and red=oxygen . 


\section{Structure 18 exo}

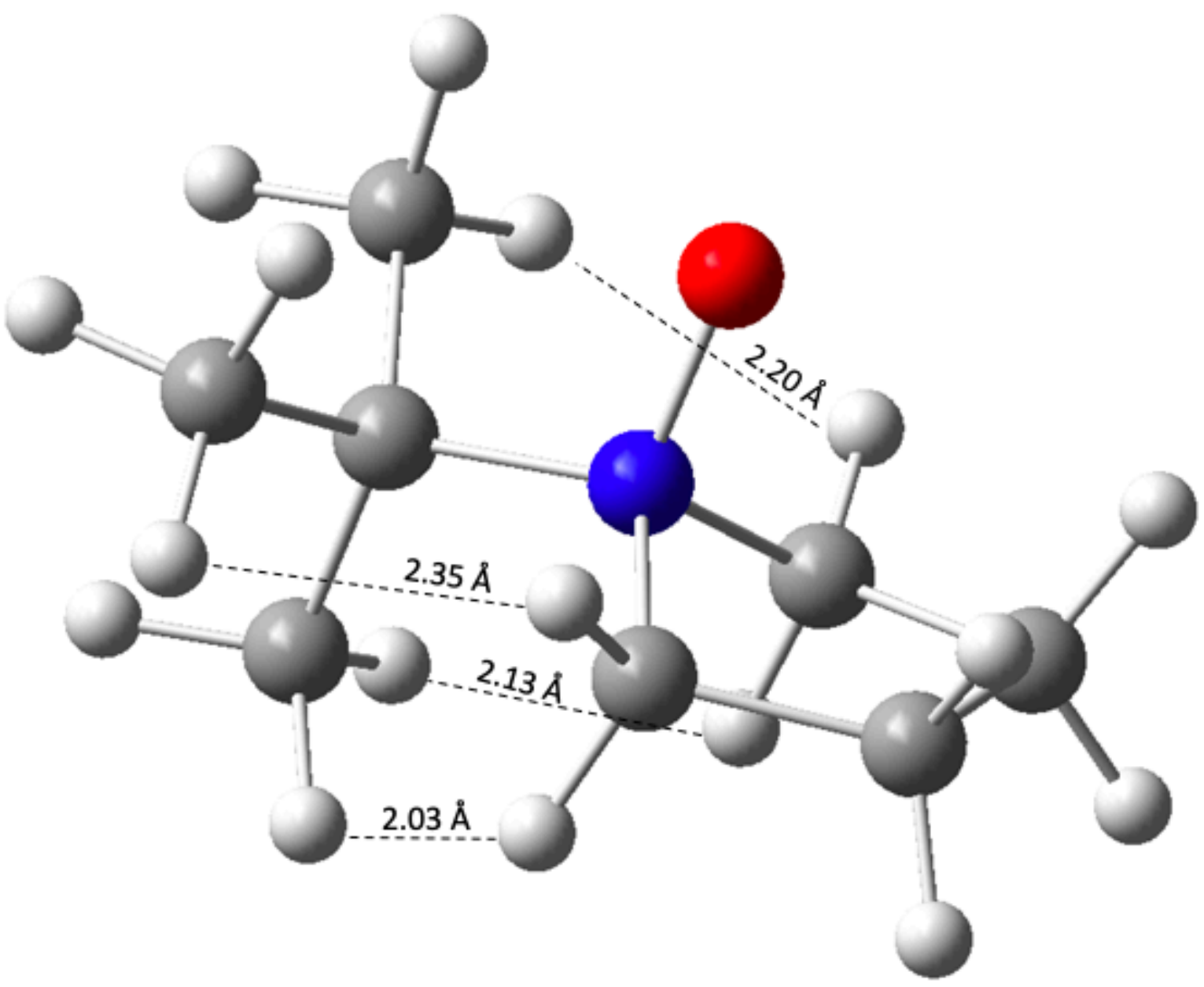

Figure S5. Ball and Stick Model for $\mathbf{1 8}_{\text {exo }}$ with key atom distances indicated. Color denotes chemical composition; grey=car- bon, white=hydrogen, blue=nitrogen, and red=oxygen . 


\section{Structure 19a}

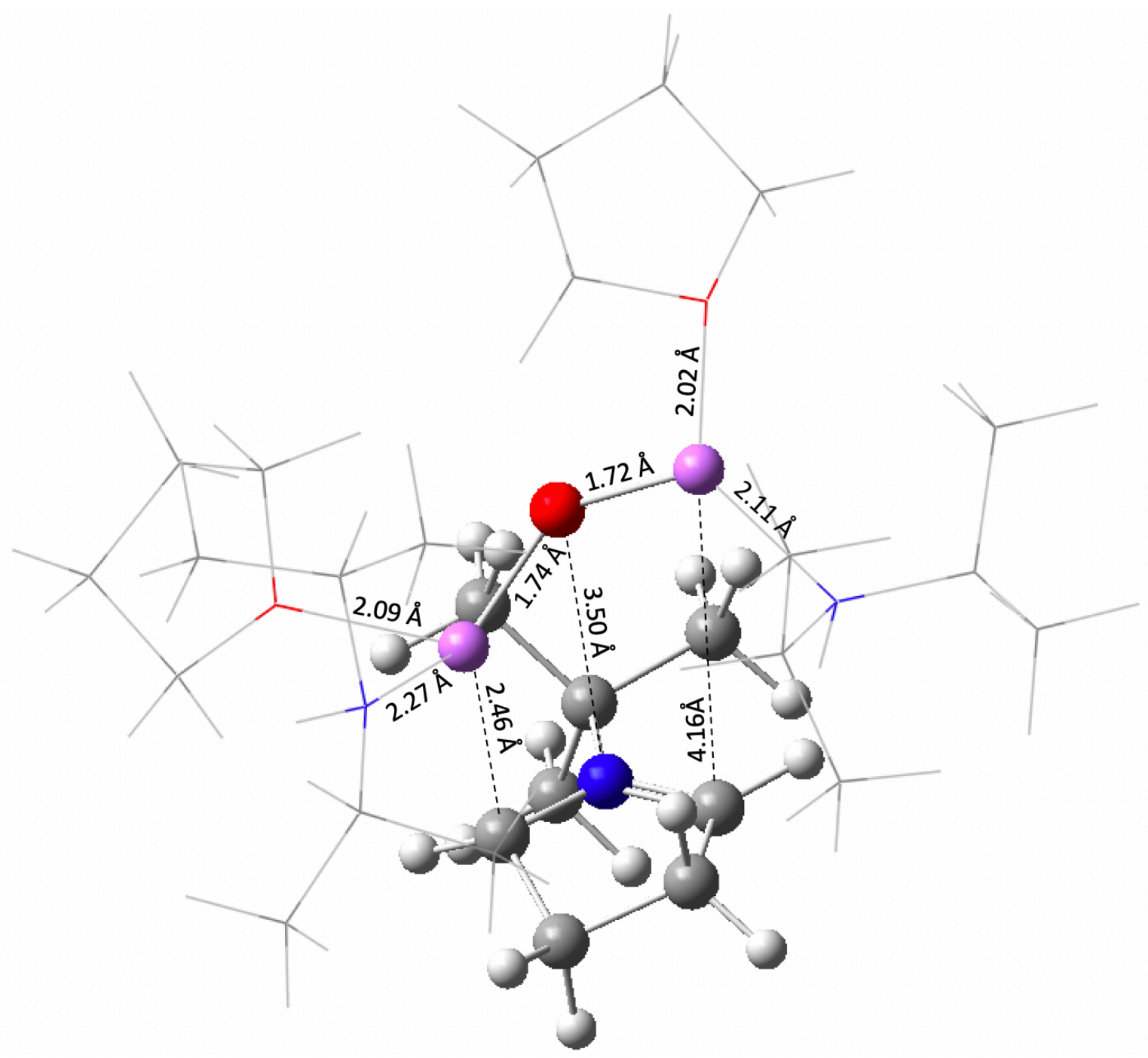

Figure S19. Ball and Stick Model for 19a with key atom distances indicated. Explicit solvent depicted in a wireframe model. Color denotes chemical composition; grey=car- bon, white $=$ hydrogen, blue=nitrogen, red=oxygen, and purple $=$ lithium . 


\section{Structure 19c}

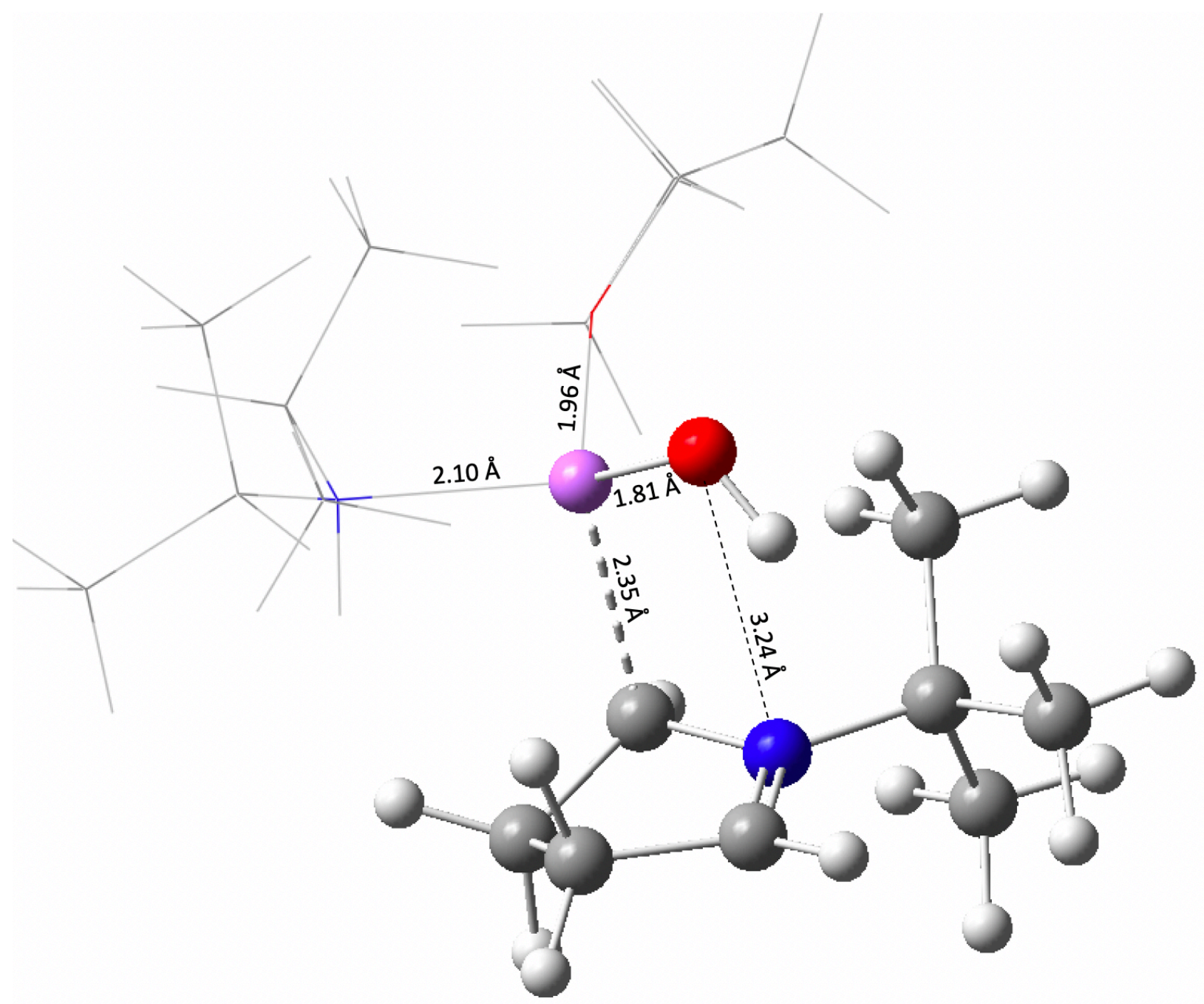

Figure S20. Ball and Stick Model for 19, with key atom distances indicated. Explicit solvent depicted in a wireframe model. Color denotes chemical composition; grey=car- bon, white $=$ hydrogen, blue $=$ nitrogen, red=oxygen, and purple $=$ lithium . 


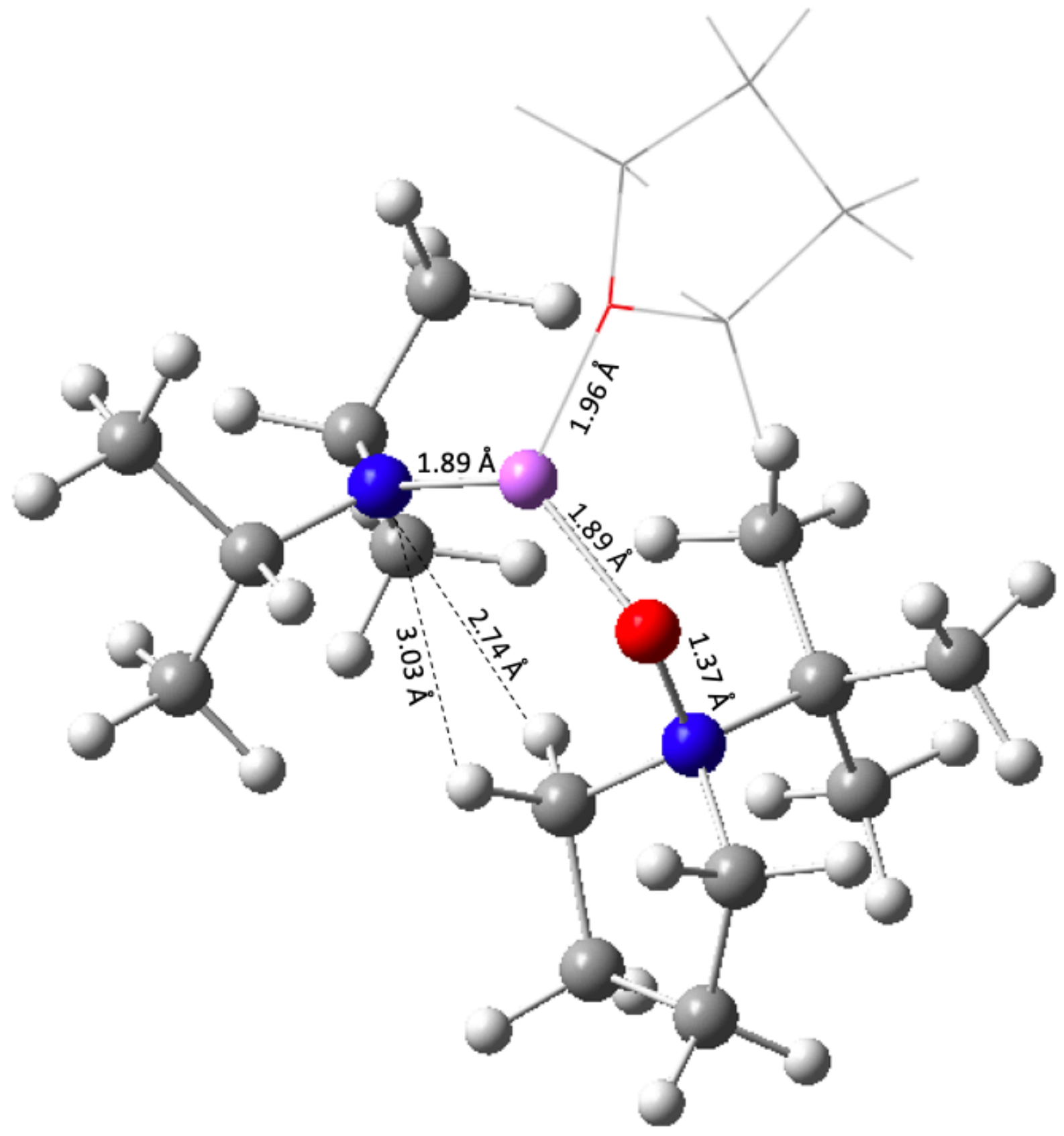

Figure S6. Ball and Stick Model for $\mathbf{2 0}_{\text {endo }}$ with key atom distances indicated. Explicit solvent depicted in a wireframe model. Color denotes chemical composition; grey=car- bon, white $=$ hydrogen, blue $=$ nitrogen, red=oxygen, and purple $=$ lithium . 


\section{Structure $20_{\text {exo }}$}

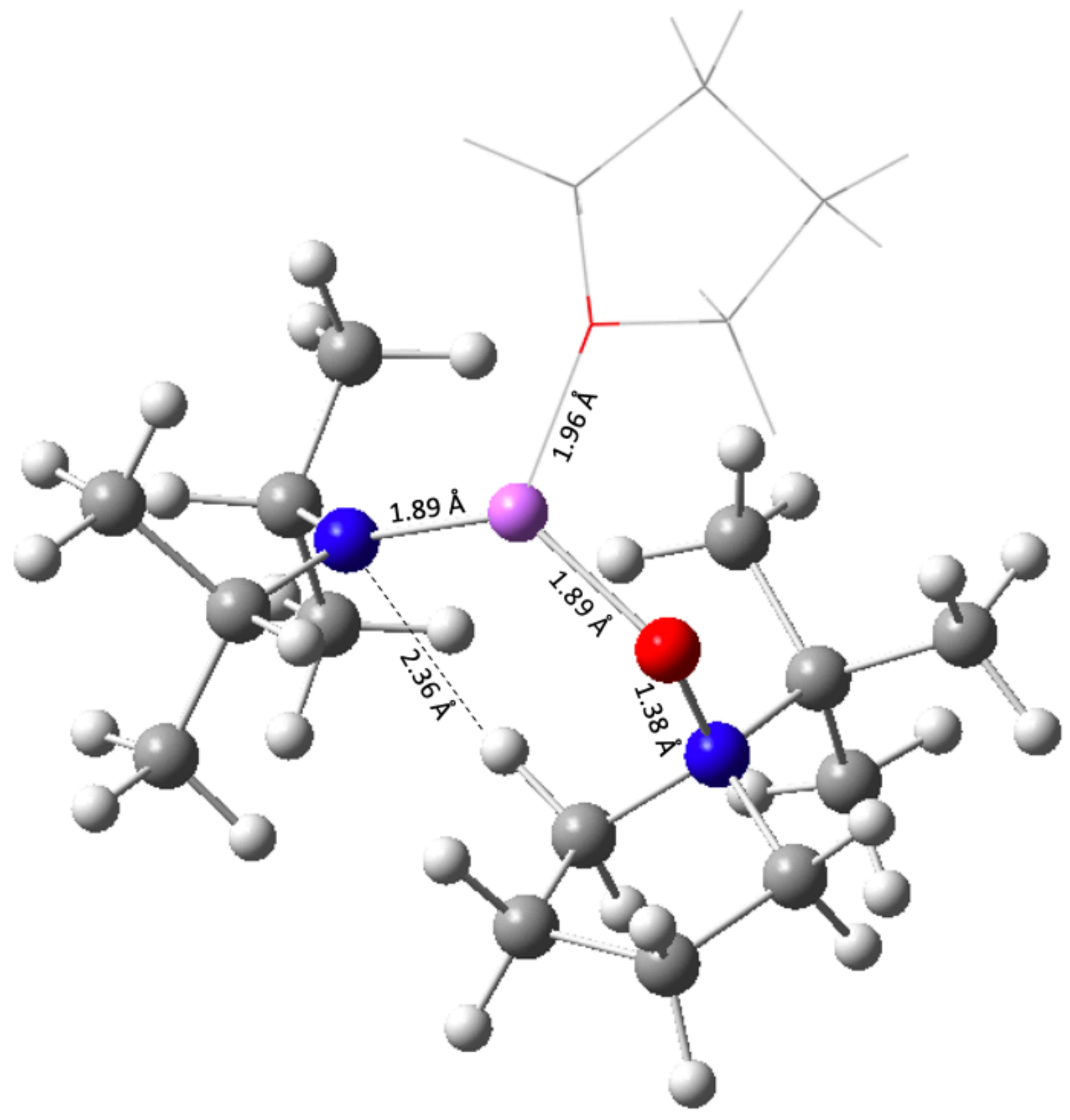

Figure S7. Ball and Stick Model for $\mathbf{2 0}_{\text {exo }}$ with key atom distances indicated. Explicit solvent depicted in a wireframe model. Color denotes chemical composition; grey=car- bon, white=hydrogen, blue=nitrogen, red=oxygen, and purple=lithium . 
Structure 20 endo-TS

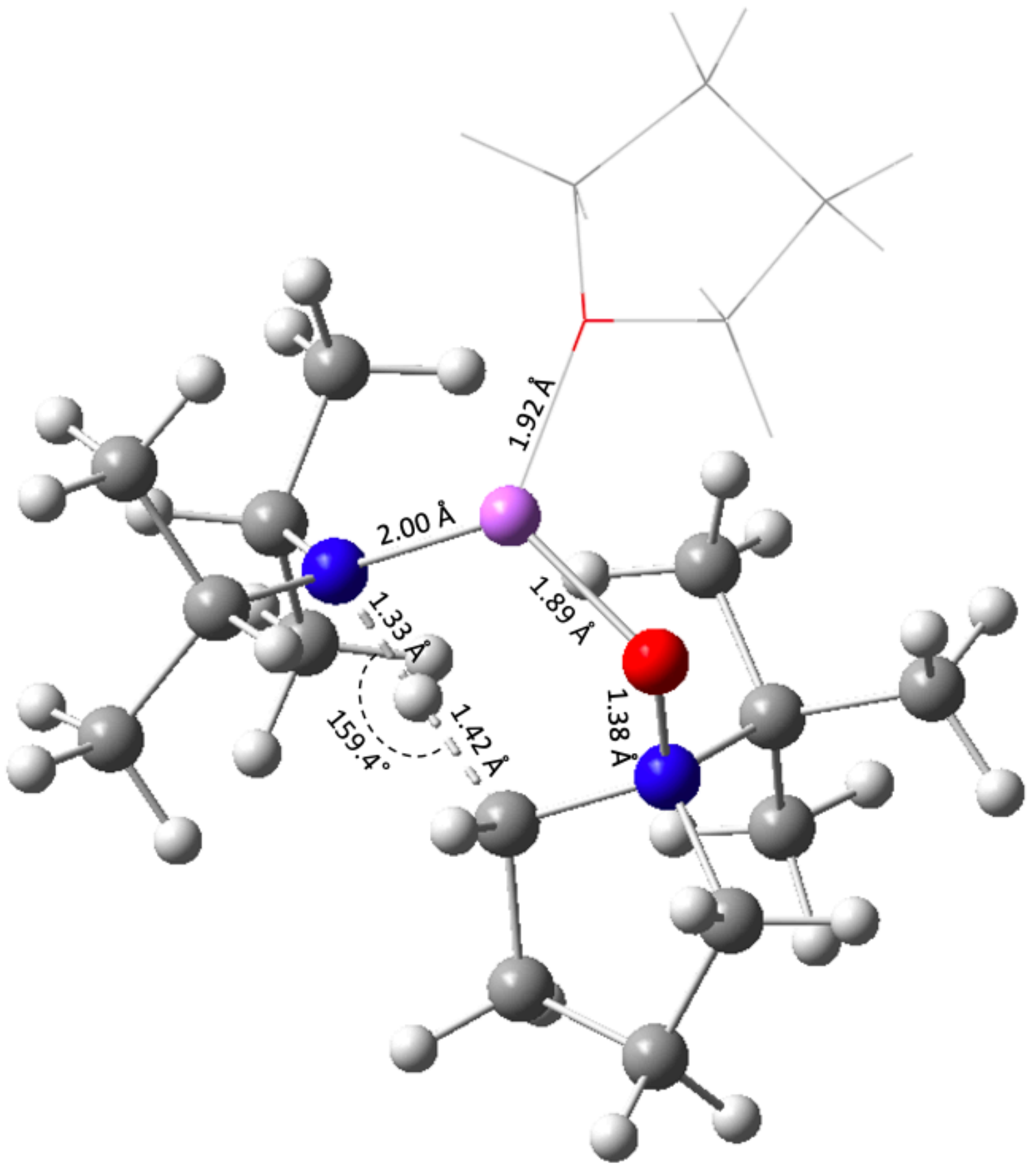

Figure S8. Ball and Stick Model for $\mathbf{2 0}_{\text {endo-Ts }}$ with key atom distances indicated. Explicit solvent depicted in a wireframe model. Color denotes chemical composition; grey=car- bon, white $=$ hydrogen, blue $=$ nitrogen, red=oxygen, and purple=lithium . 


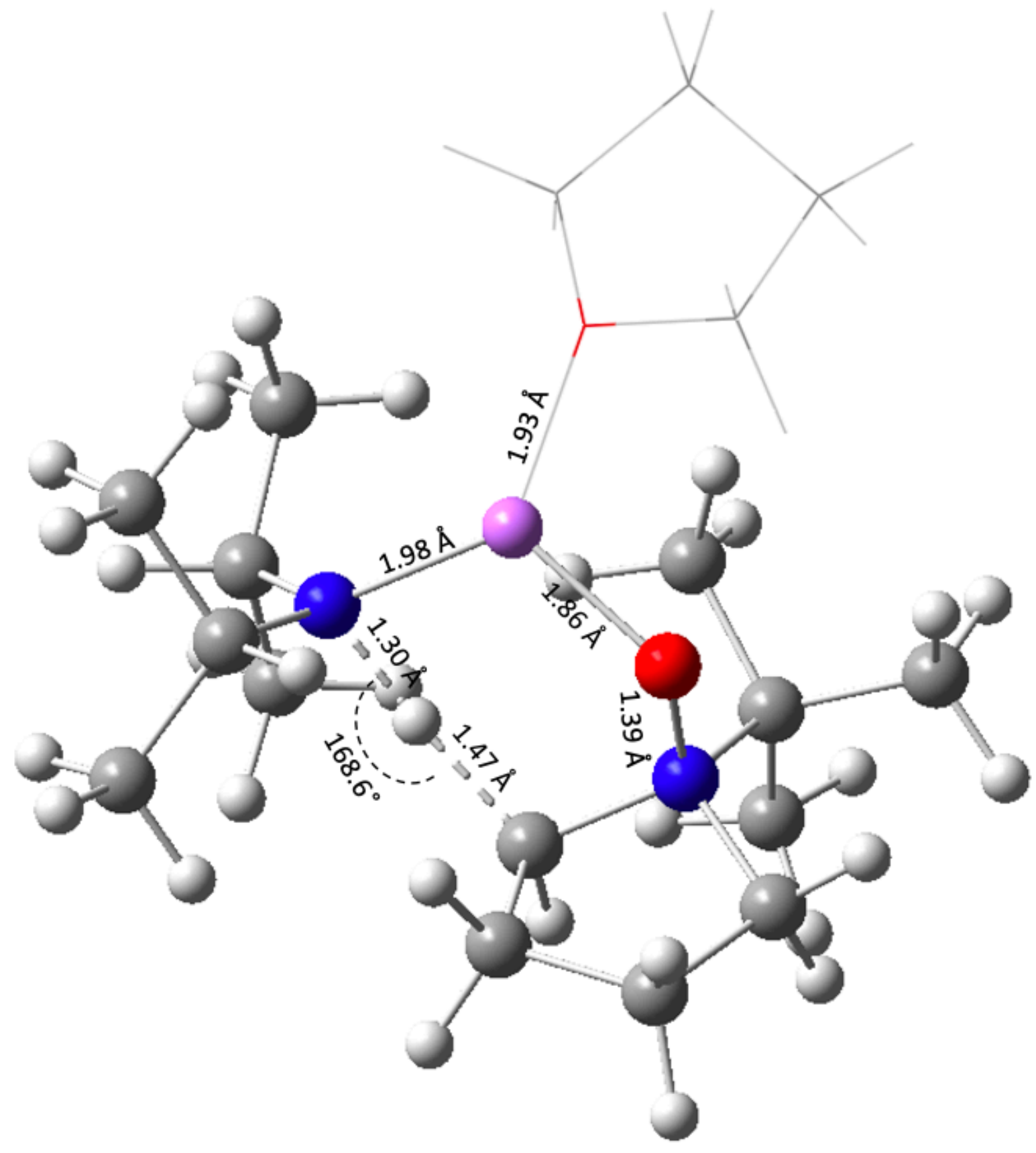

Figure S9. Ball and Stick Model for $\mathbf{2 0}_{\text {exo-Ts }}$ with key atom distances and angles indicated. Explicit solvent depicted in a wireframe model. Color denotes chemical composition; grey $=$ car- bon, white=hydrogen, blue=nitrogen, red=oxygen, and purple=lithium. 


\section{Structure 21 endo}

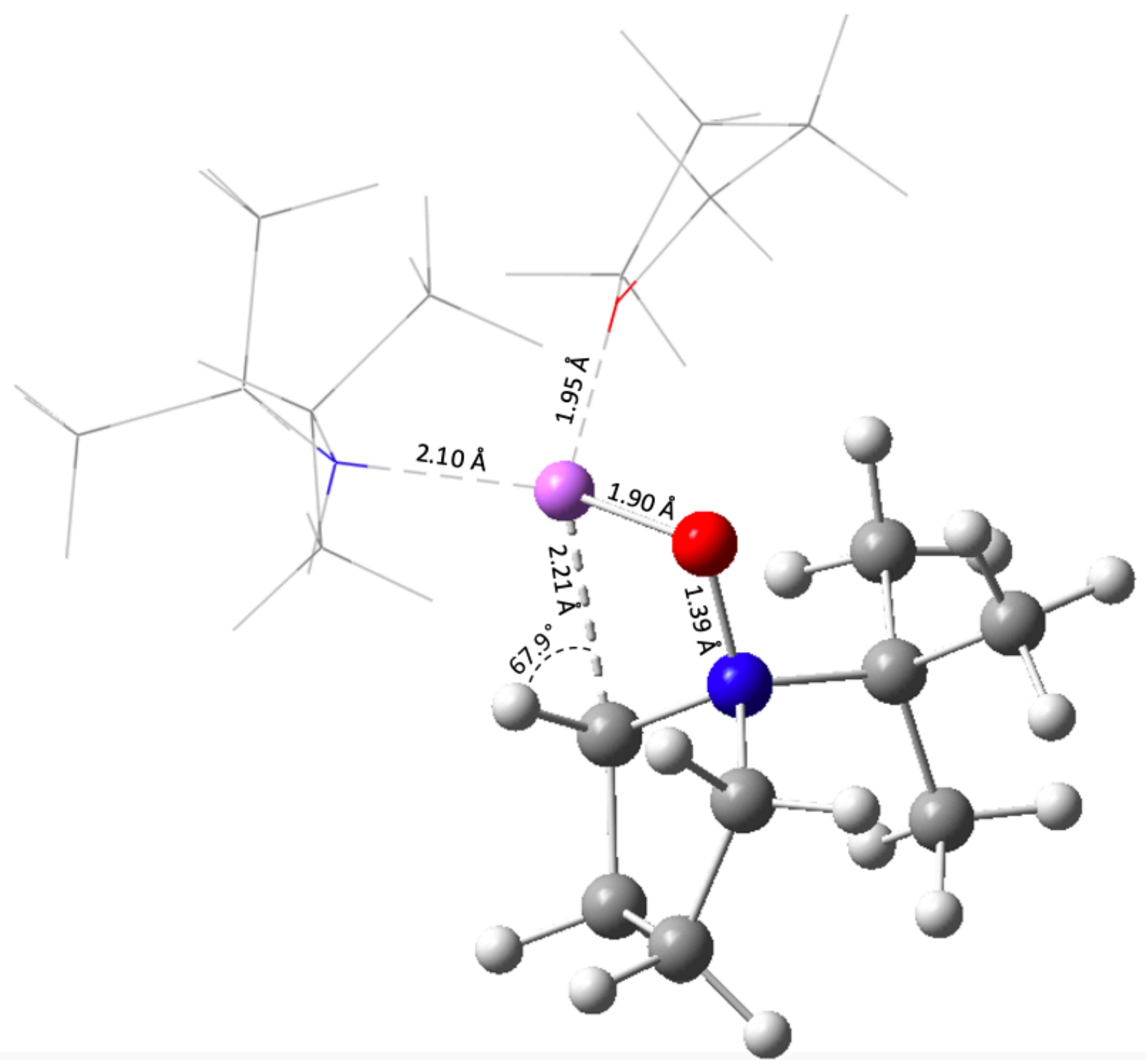

Figure S10. Ball and Stick Model for $\mathbf{2 1}_{\text {endo }}$ with key atom distances and angles indicated. Explicit solvent depicted in a wireframe model. Color denotes chemical composition; grey $=$ car- bon, white=hydrogen, blue=nitrogen, red=oxygen, and purple=lithium . 


\section{Structure 21 exo}

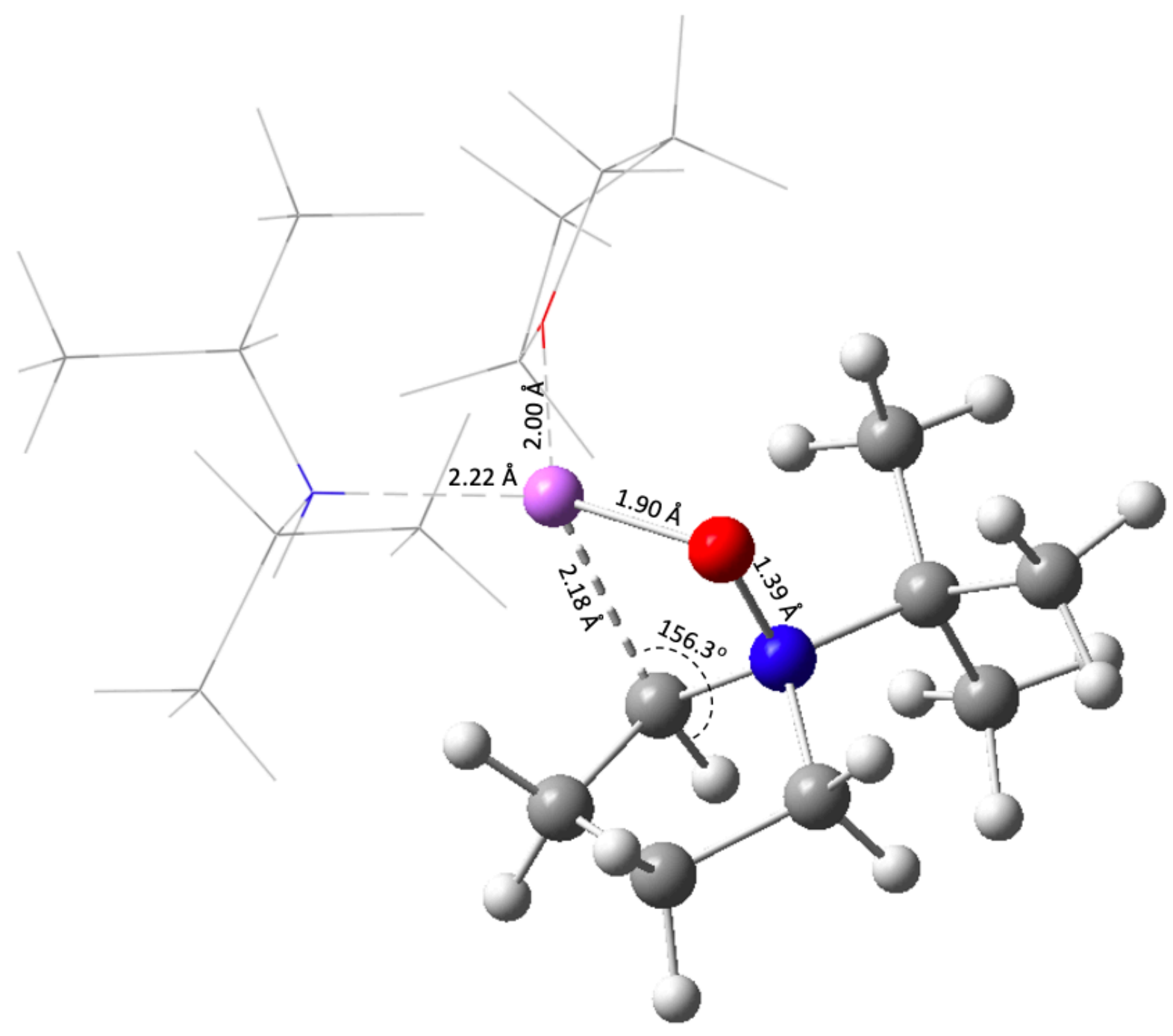

Figure S11. Ball and Stick Model for $\mathbf{2 1}_{\text {exo }}$ with key atom distances and angles indicated. Explicit solvent depicted in a wireframe model. Color denotes chemical composition; grey $=$ car- bon, white=hydrogen, blue=nitrogen, red=oxygen, and purple=lithium . 


\section{Structure $21_{\text {exo-m }}$}

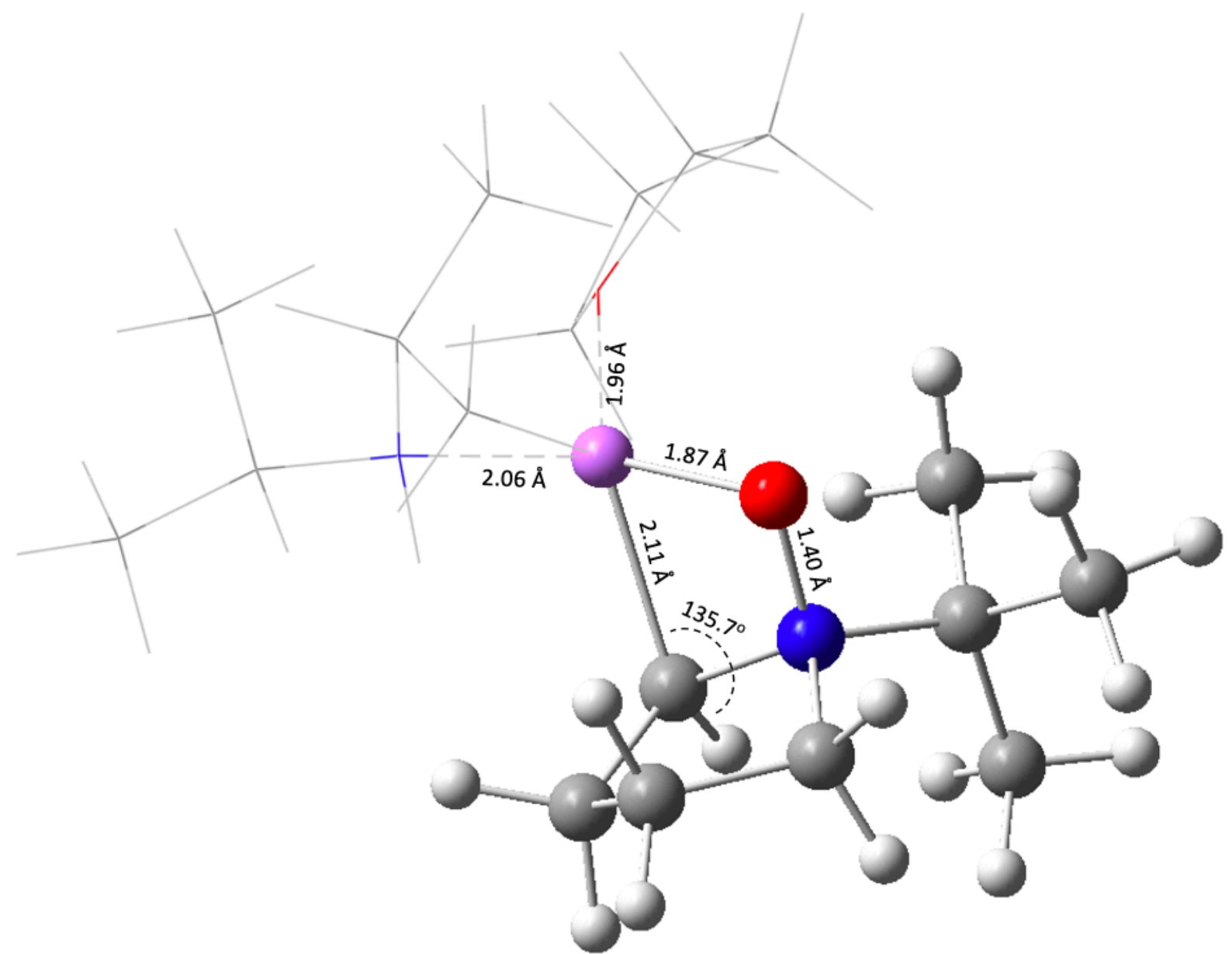

Figure S12. Ball and Stick Model for $\mathbf{2 1}_{\text {exo-m }}$ with key atom distances and angles indicated. Explicit solvent depicted in a wireframe model. Color denotes chemical composition; grey=car- bon, white=hydrogen, blue=nitrogen, red=oxygen, and purple=lithium . 
Structure $21_{\text {TS }}$

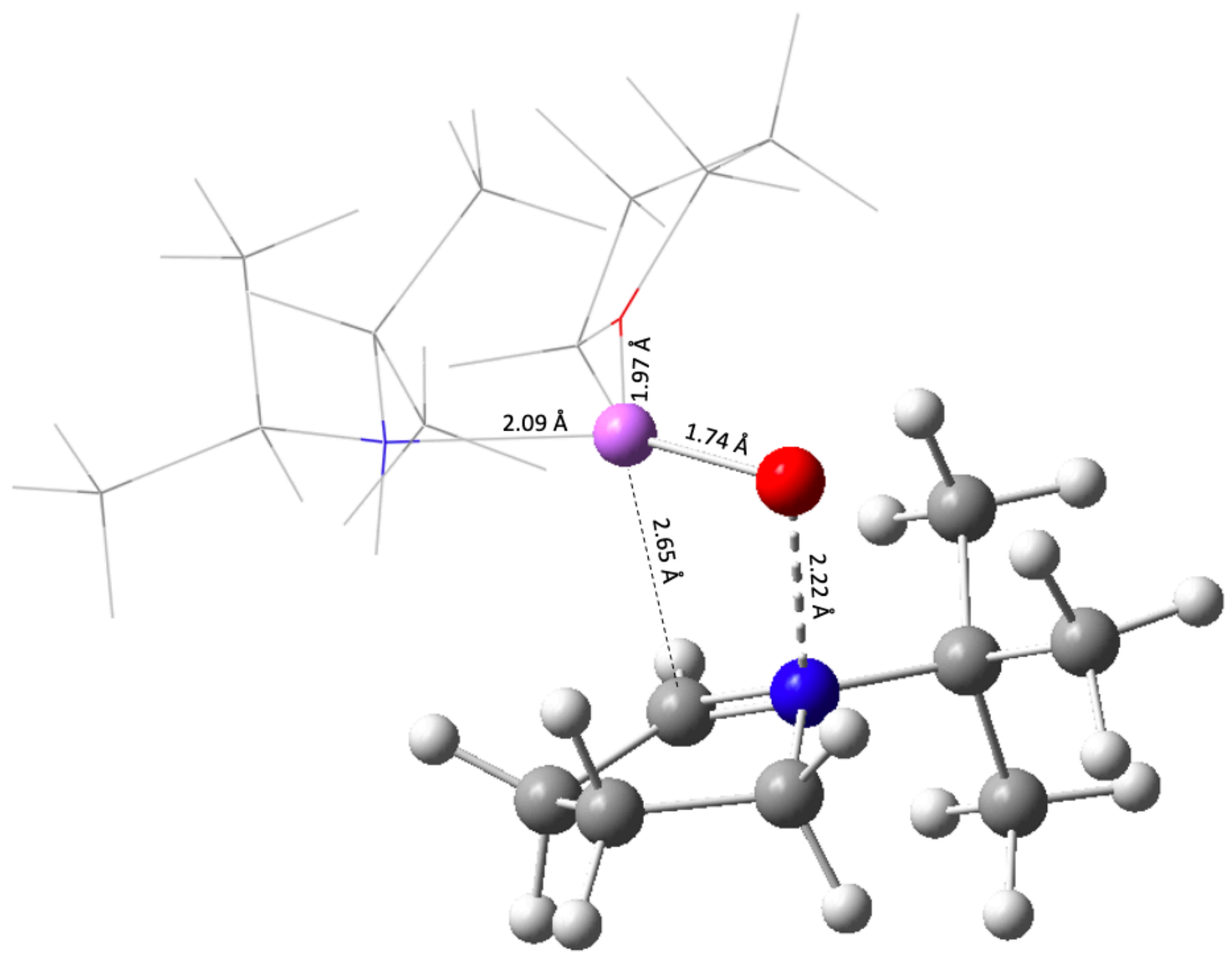

Figure S13. Ball and Stick Model for $\mathbf{2 1}_{\mathrm{TS}}$ with key atom distances indicated. Explicit solvent depicted in a wireframe model. Color denotes chemical composition; grey=car- bon, white $=$ hydrogen, blue=nitrogen, red $=$ oxygen, and purple=lithium . 


\section{Structure 22}

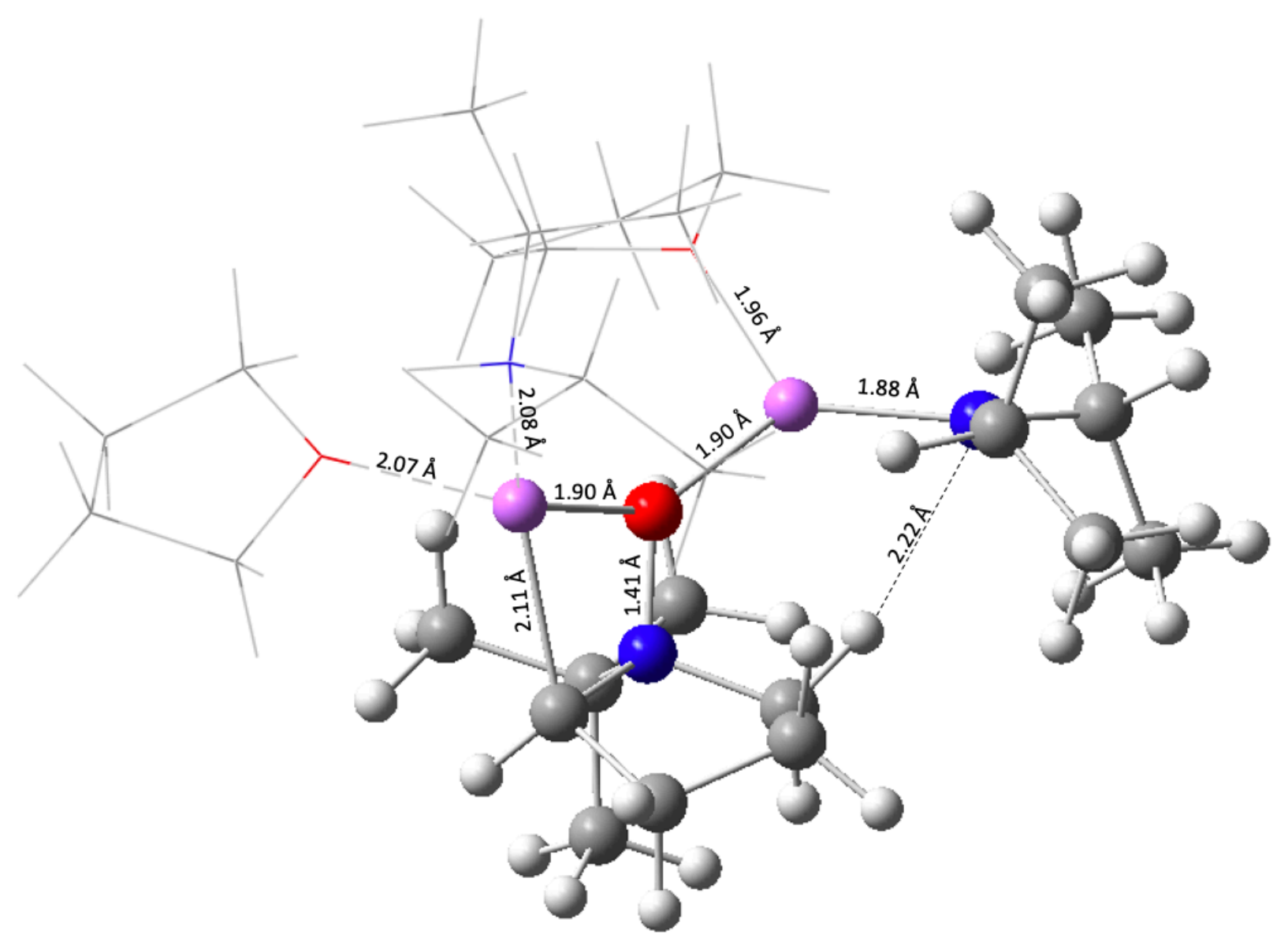

Figure S14. Ball and Stick Model for 22 with key atom distances indicated. Explicit solvent depicted in a wireframe model. Color denotes chemical composition; grey=car- bon, white=hydrogen, blue=nitrogen, red=oxygen, and purple=lithium . 
Structure 22 2 TS-A

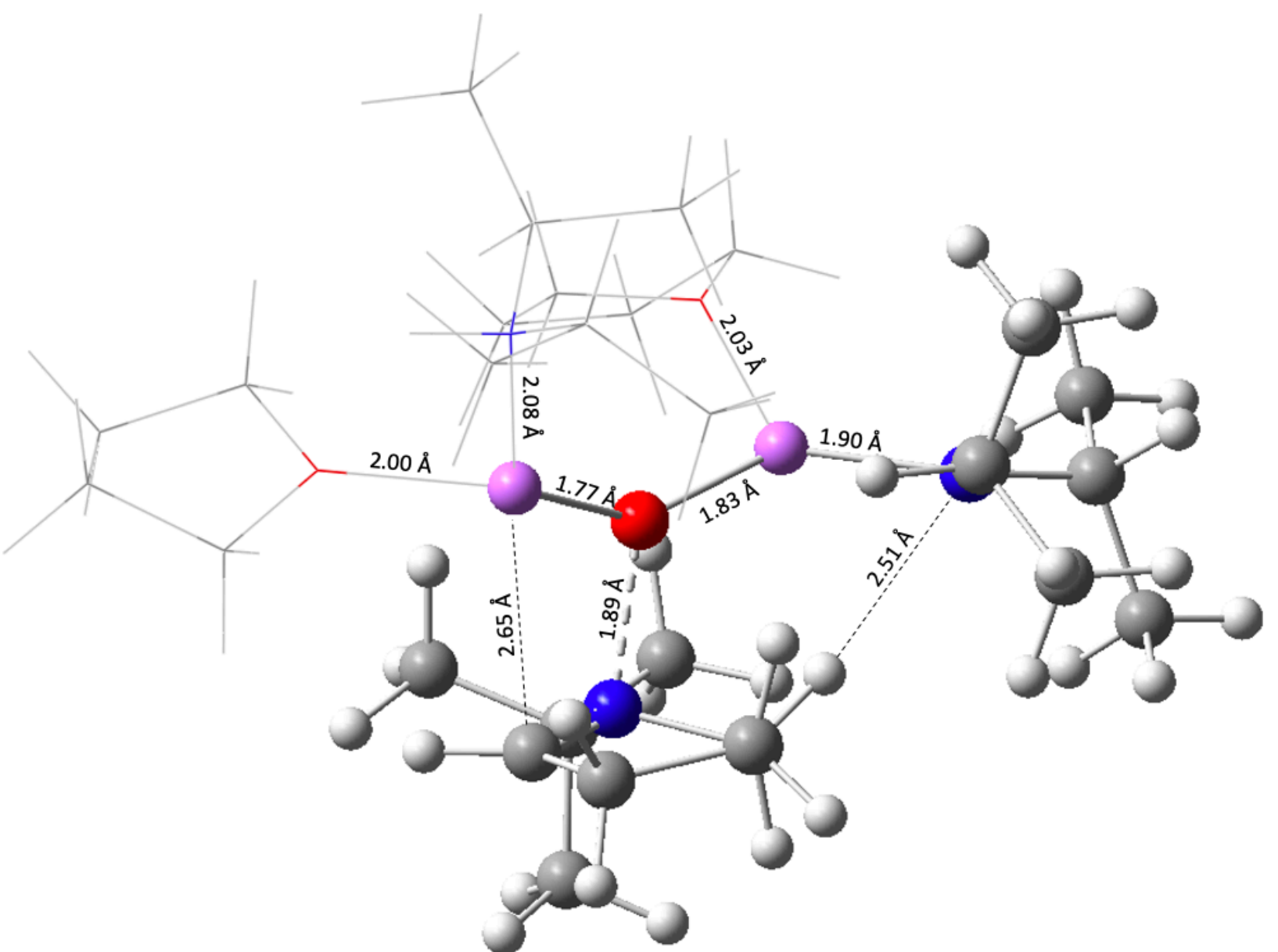

Figure S15. Ball and Stick Model for $\mathbf{2 2}_{\mathrm{TS}-\mathrm{A}}$ with key atom distances indicated. Explicit solvent depicted in a wireframe model. Color denotes chemical composition; grey=car- bon, white=hydrogen, blue $=$ nitrogen, red=oxygen, and purple=lithium . 


\section{Structure $22_{\mathrm{TS}-\mathrm{B}}$}

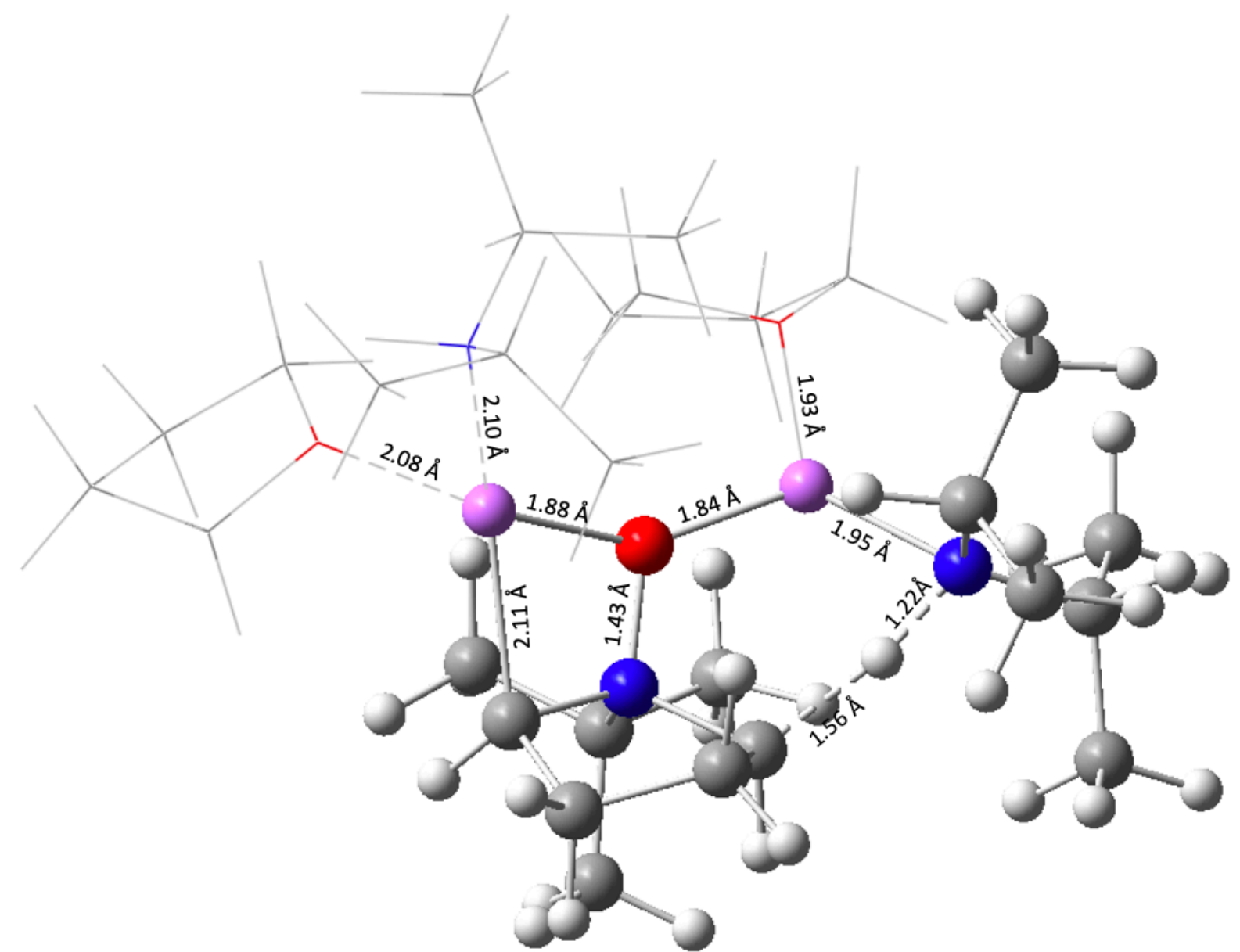

Figure S16. Ball and Stick Model for $\mathbf{2 2}_{\mathrm{TS}-\mathrm{B}}$ with key atom distances indicated. Explicit solvent depicted in a wireframe model. Color denotes chemical composition; grey=car- bon, white=hydrogen, blue=nitrogen, red=oxygen, and purple=lithium . 


\section{Structure 23}

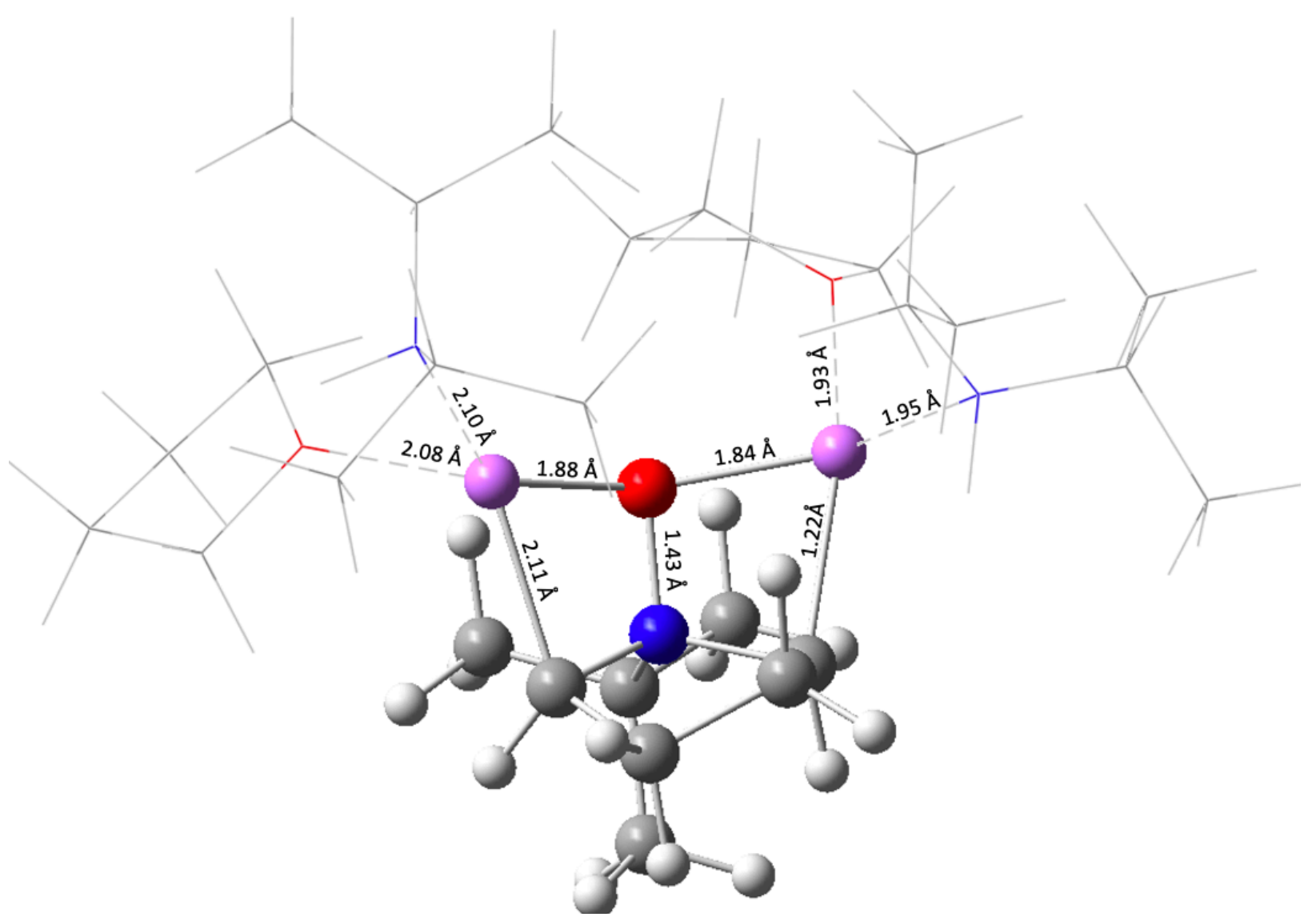

Figure S17. Ball and Stick Model for 23 with key atom distances indicated. Explicit solvent depicted in a wireframe model. Color denotes chemical composition; grey=car- bon, white=hydrogen, blue=nitrogen, red=oxygen, and purple=lithium . 


\section{Structure $23_{\mathrm{TS}}$}

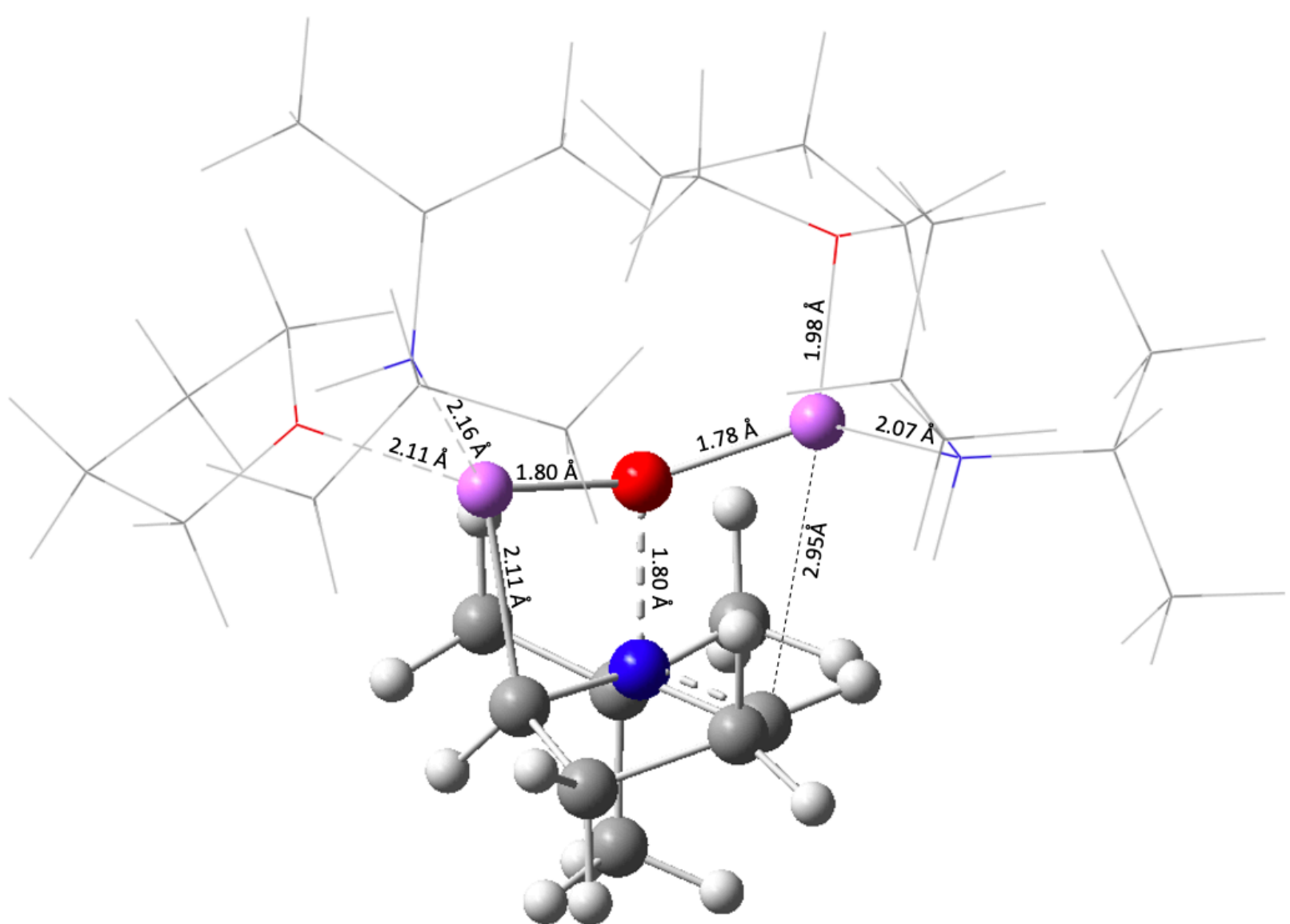

Figure S18. Ball and Stick Model for $\mathbf{2 3}_{\mathrm{TS}}$ with key atom distances indicated. Explicit solvent depicted in a wireframe model. Color denotes chemical composition; grey=car- bon, white=hydrogen, blue=nitrogen, red=oxygen, and purple $=$ lithium . 


\section{Structure 28}

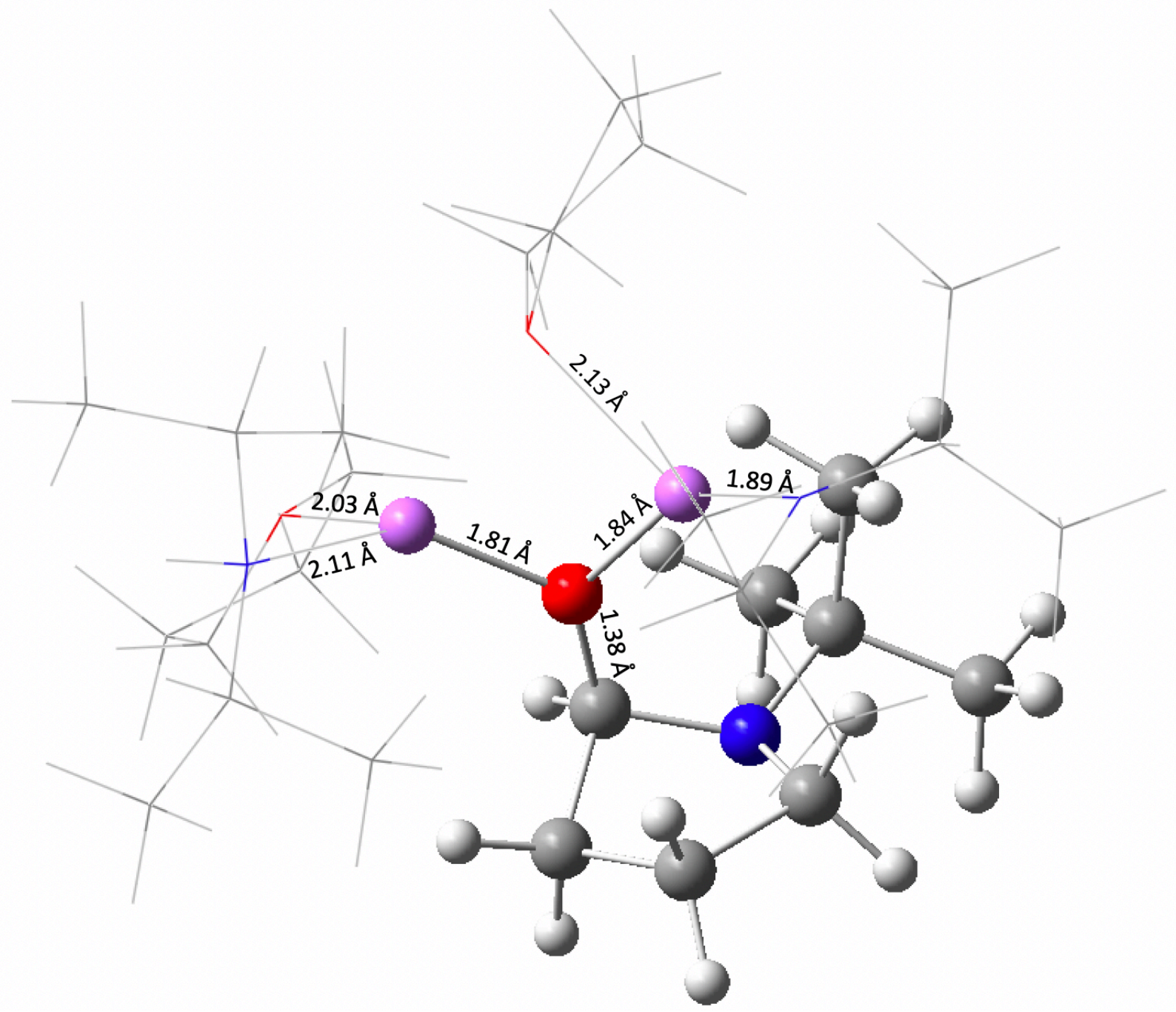

Figure S21. Ball and Stick Model for 28 with key atom distances indicated. Explicit solvent depicted in a wireframe model. Color denotes chemical composition; grey=car- bon, white $=$ hydrogen, blue $=$ nitrogen, red=oxygen, and purple=lithium . 


\section{Gaussian Outputs for Structures of N-Oxide to 1,3-dipole Mechanism Structure 18 endo - M06-2x/jul-cc-pVDZ}

Input

$\#$ 062x/jul-cc-pvDz opt=(calcfc,noeigen,z-matrix $)$ optcyc $=50$ freq $\mathrm{scrf}=(\mathrm{pcm}, \mathrm{solvent}=\mathrm{THF})$

Optimized Structure:

\begin{tabular}{|c|c|c|c|c|}
\hline \multirow{3}{*}{$\begin{array}{l}\text { Center } \\
\text { Number } \\
1\end{array}$} & \multirow{2}{*}{$\begin{array}{l}\text { Atomic } \\
\text { Number }\end{array}$} & \multirow{2}{*}{$\begin{array}{l}\text { Atomic } \\
\text { Type }\end{array}$} & \multicolumn{2}{|c|}{ Coordinates (Angstroms) } \\
\hline & & & $\mathrm{X}$ & $\mathrm{Z}$ \\
\hline & 7 & 0.000000 & 0.000000 & 0.000000 \\
\hline 2 & 8 & 0.000000 & 0.000000 & 1.366491 \\
\hline 3 & 6 & 1.474839 & 0.000000 & -0.493662 \\
\hline 4 & 6 & -0.810323 & -1.175992 & -0.508593 \\
\hline 5 & 6 & -0.789830 & 1.181564 & -0.525303 \\
\hline 6 & 6 & -1.474693 & -0.777464 & -1.825202 \\
\hline 7 & 6 & -1.453911 & 0.770122 & -1.839490 \\
\hline 8 & 1 & -1.549671 & -1.311679 & 0.284501 \\
\hline 9 & 1 & -0.183228 & -2.066600 & -0.554609 \\
\hline 10 & 1 & -2.497992 & -1.165667 & -1.852506 \\
\hline 11 & 1 & -0.952211 & -1.189871 & -2.693969 \\
\hline 12 & 1 & -2.466013 & 1.184682 & -1.886236 \\
\hline 13 & 1 & -0.908902 & 1.146343 & -2.710250 \\
\hline 14 & 1 & -1.526408 & 1.350921 & 0.264028 \\
\hline 15 & 1 & -0.142526 & 2.057481 & -0.593986 \\
\hline 16 & 6 & 2.110870 & -1.333180 & -0.099161 \\
\hline 17 & 6 & 1.580900 & 0.203089 & -2.003777 \\
\hline 18 & 6 & 2.209483 & 1.126797 & 0.231697 \\
\hline 19 & 1 & 1.852251 & -2.141458 & -0.793708 \\
\hline 20 & 1 & 3.200949 & -1.216939 & -0.123687 \\
\hline 21 & 1 & 1.809159 & -1.604081 & 0.917831 \\
\hline 22 & 1 & 1.025844 & -0.552172 & -2.570207 \\
\hline 23 & 1 & 1.258801 & 1.204343 & -2.311894 \\
\hline 24 & 1 & 2.638991 & 0.099986 & -2.275446 \\
\hline 25 & 1 & 2.243292 & 0.928740 & 1.305343 \\
\hline 26 & 1 & 3.231980 & 1.169746 & -0.164272 \\
\hline 27 & 1 & 1.748837 & 2.107352 & 0.067133 \\
\hline
\end{tabular}

Thermodynamics:

Zero-point correction $=$

Thermal correction to Energy=

Thermal correction to Enthalpy=

Thermal correction to Gibbs Free Energy=

Sum of electronic and zero-point Energies=

Sum of electronic and thermal Energies=

Sum of electronic and thermal Enthalpies=
0.248384 (Hartree/Particle)

0.258806

0.259751

0.213813

$-444.592092$

$-444.581670$

$-444.580725$ 


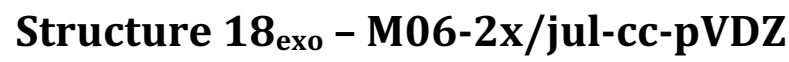

Input:

$\#$ 062x/jul-cc-pvDz opt=(calcfc,noeigen,z-matrix $)$ optcyc $=50$ freq $\mathrm{scrf}=(\mathrm{pcm}$,solvent=THF $)$

Optimized Structure:

\begin{tabular}{|c|c|c|c|c|}
\hline \multirow{2}{*}{$\begin{array}{l}\text { Center } \\
\text { Number }\end{array}$} & Atomic & \multirow{2}{*}{$\begin{array}{l}\text { Atomic } \\
\text { Type }\end{array}$} & \multicolumn{2}{|c|}{ Coordinates (Angstrom } \\
\hline & Number & & $\mathrm{X}$ & $\mathrm{Z}$ \\
\hline 1 & 7 & 0.000000 & 0.000000 & 0.000000 \\
\hline 2 & 8 & 0.000000 & 0.000000 & 1.367666 \\
\hline 3 & 6 & 1.446982 & 0.000000 & -0.540509 \\
\hline 4 & 6 & -0.825081 & -1.161225 & -0.482343 \\
\hline 5 & 6 & -0.810760 & 1.179700 & -0.465170 \\
\hline 6 & 6 & -2.247250 & -0.769586 & -0.094348 \\
\hline 7 & 6 & -2.260153 & 0.775647 & -0.176382 \\
\hline 8 & 1 & -0.726447 & -1.238997 & -1.570315 \\
\hline 9 & 1 & -0.453574 & -2.066227 & 0.001902 \\
\hline 10 & 1 & -2.971165 & -1.230636 & -0.774529 \\
\hline 11 & 1 & -2.467739 & -1.102410 & 0.922913 \\
\hline 12 & 1 & -2.918230 & 1.138723 & -0.973002 \\
\hline 13 & 1 & -2.600845 & 1.209900 & 0.767293 \\
\hline 14 & 1 & -0.641395 & 1.335982 & -1.536111 \\
\hline 15 & 1 & -0.469257 & 2.047795 & 0.099867 \\
\hline 16 & 6 & 2.208959 & -1.116307 & 0.168490 \\
\hline 17 & 6 & 1.500036 & -0.228725 & -2.053190 \\
\hline 18 & 6 & 2.065644 & 1.351181 & -0.189195 \\
\hline 19 & 1 & 1.743890 & -2.095486 & 0.002368 \\
\hline 20 & 1 & 3.224287 & -1.151897 & $7-0.246387$ \\
\hline 21 & 1 & 2.261867 & -0.923696 & $5 \quad 1.242664$ \\
\hline 22 & 1 & 1.264545 & -1.264437 & $7 \quad-2.322514$ \\
\hline 23 & 1 & 0.843030 & 0.445695 & -2.614663 \\
\hline 24 & 1 & 2.527527 & -0.028955 & $5-2.381815$ \\
\hline 25 & 1 & 1.866628 & 1.590964 & 0.860461 \\
\hline 26 & 1 & 3.150221 & 1.289633 & -0.339136 \\
\hline 27 & 1 & 1.682600 & 2.154465 & -0.830818 \\
\hline
\end{tabular}

Thermodynamics:

Zero-point correction $=$

Thermal correction to Energy=

Thermal correction to Enthalpy=

Thermal correction to Gibbs Free Energy=

Sum of electronic and zero-point Energies=

Sum of electronic and thermal Energies=

Sum of electronic and thermal Enthalpies=

Sum of electronic and thermal Free Energies=
0.248036 (Hartree/Particle)

$$
0.258637
$$$$
0.259581
$$

0.213199

$-444.599170$

$-444.588569$

$-444.587625$

$-444.634007$ 
Structure 19a - M06-2x/jul-cc-pVDZ

Input:

$\# \mathrm{~m} 062 \mathrm{x} / \mathrm{jul}-\mathrm{cc}-\mathrm{pvDz}$ opt=(calcfc,noeigen $)$ optcyc=50 SCF=XQC scrf $=(\mathrm{pcm}$,so lvent $=\mathrm{THF}$,read)

PCM adjusted for no symmetry (NonSymmPCM)

Optimized Structure:

\begin{tabular}{|c|c|c|c|c|}
\hline \multirow{3}{*}{$\begin{array}{l}\text { Center } \\
\text { Number } \\
1\end{array}$} & \multirow{2}{*}{$\begin{array}{l}\text { Atomic } \\
\text { Number }\end{array}$} & \multirow{2}{*}{$\begin{array}{l}\text { Atomic } \\
\text { Type }\end{array}$} & \multicolumn{2}{|c|}{ Coordinates (Angstroms) } \\
\hline & & & $\mathrm{X}$ & $\mathrm{Z}$ \\
\hline & 7 & 1.260826 & 2.869329 & -0.409991 \\
\hline 2 & 8 & -0.143361 & -0.275878 & -1.028527 \\
\hline 3 & 6 & 1.603410 & 3.011613 & -1.861841 \\
\hline 4 & 6 & 0.159688 & 3.338387 & 0.140728 \\
\hline 5 & 6 & 2.113217 & 2.237082 & 0.480359 \\
\hline 6 & 6 & 0.159602 & 2.919347 & 1.580541 \\
\hline 7 & 6 & 1.663519 & 2.752604 & 1.833454 \\
\hline 8 & 1 & -2.130622 & 1.788704 & 0.564362 \\
\hline 9 & 1 & -0.673023 & 3.696697 & -0.449597 \\
\hline 10 & 1 & -0.308347 & 1.912389 & 1.648926 \\
\hline 11 & 1 & -0.358928 & 3.603894 & 2.259473 \\
\hline 12 & 1 & 1.877192 & 2.091023 & 2.677785 \\
\hline 13 & 1 & 2.111402 & 3.747329 & 2.043132 \\
\hline 14 & 1 & 3.163954 & 2.259442 & 0.193695 \\
\hline 15 & 6 & 0.384968 & 3.471313 & -2.658214 \\
\hline 16 & 6 & 2.706868 & 4.069287 & -1.961118 \\
\hline 17 & 6 & 2.074492 & 1.653003 & -2.380534 \\
\hline 18 & 1 & 0.038313 & 4.462363 & -2.340300 \\
\hline 19 & 1 & 0.680653 & 3.542136 & -3.711355 \\
\hline 20 & 1 & -0.436679 & 2.749829 & -2.580047 \\
\hline 21 & 1 & 2.364566 & 5.026208 & -1.545959 \\
\hline 22 & 1 & 3.611101 & 3.751939 & -1.429271 \\
\hline 23 & 1 & 2.962423 & 4.217271 & -3.017814 \\
\hline 24 & 1 & 3.025333 & 1.369026 & -1.914508 \\
\hline 25 & 1 & 1.311440 & 0.885362 & -2.151645 \\
\hline 26 & 1 & 2.235192 & 1.734075 & -3.464403 \\
\hline 27 & 3 & 1.168740 & 0.005413 & 0.079921 \\
\hline 28 & 8 & 2.885092 & -0.993680 & -0.566461 \\
\hline 29 & 6 & 4.286727 & -0.713824 & -0.478708 \\
\hline 30 & 6 & 4.942843 & -1.461777 & -1.640594 \\
\hline 31 & 6 & 3.801077 & -1.538103 & -2.659693 \\
\hline 32 & 6 & 2.597893 & -1.757438 & -1.753182 \\
\hline 33 & 1 & 4.431987 & 0.373568 & -0.559463 \\
\hline 34 & 1 & 4.656089 & -1.040180 & 0.504290 \\
\hline 35 & 1 & 5.833856 & -0.945823 & -2.014086 \\
\hline 36 & 1 & 5.233323 & -2.472828 & -1.325928 \\
\hline 37 & 1 & 3.701510 & -0.589163 & -3.202537 \\
\hline
\end{tabular}




\begin{tabular}{|c|c|c|c|c|c|}
\hline 38 & 1 & 0 & 3.929194 & -2.347029 & -3.387209 \\
\hline 39 & 1 & 0 & 1.629731 & -1.392531 & -2.118915 \\
\hline 40 & 1 & 0 & 2.508935 & -2.821526 & -1.473749 \\
\hline 41 & 7 & 0 & 1.483533 & -1.310373 & 1.907515 \\
\hline 42 & 1 & 0 & 2.429983 & -1.189433 & 1.554277 \\
\hline 43 & 6 & 0 & 1.008532 & -2.580758 & 1.315748 \\
\hline 44 & 6 & 0 & 1.542543 & -1.252389 & 3.37893 \\
\hline 45 & . & 0 & 0.857249 & -2.354672 & 0.25004 \\
\hline 46 & 6 & ) & 2.046619 & -3.696133 & 1.444039 \\
\hline 47 & 6 & 0 & -0.345008 & -2.999010 & 1.874621 \\
\hline 48 & 1 & 0 & 1.520938 & -2.271883 & 3.80080 \\
\hline 49 & 6 & 0 & 2.862473 & -0.613220 & 3.79889 \\
\hline 50 & & 0 & 0.350735 & -0.480220 & 3.93978 \\
\hline 51 & . & 0 & 2.991072 & -3.386079 & 0.97348 \\
\hline 52 & 1 & 0 & 2.246898 & -3.941160 & 2.49749 \\
\hline 53 & 1 & 0 & 1.698787 & -4.611210 & 0.94517 \\
\hline 54 & . & 0 & -1.089665 & -2.208923 & 1.719123 \\
\hline 55 & 1 & 0 & -0.690801 & -3.899959 & 1.351758 \\
\hline 56 & 1 & 0 & -0.294434 & -3.233966 & 2.94688 \\
\hline 57 & 1 & 0 & 3.709549 & -1.250528 & 3.50943 \\
\hline 58 & 1 & 0 & 2.985118 & 0.362972 & 3.30816 \\
\hline 59 & 1 & 0 & 2.899852 & -0.461189 & 4.88475 \\
\hline 60 & 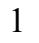 & 0 & 0.341310 & 0.541916 & 3.53291 \\
\hline 61 & 1 & 0 & -0.595933 & -0.961949 & 3.667286 \\
\hline 62 & 1 & 0 & 0.405419 & -0.424520 & 5.035841 \\
\hline 63 & 7 & 0 & -2.823221 & 1.041357 & 0.579541 \\
\hline 64 & 6 & ) & -4.155221 & 1.628376 & 0.32611 \\
\hline 65 & & 0 & -2.651919 & 0.323518 & 1.86015 \\
\hline 66 & 1 & 0 & -4.743396 & 1.630839 & 1.25733 \\
\hline 67 & 6 & 0 & -3.990823 & 3.072240 & -0.13497 \\
\hline 68 & 6 & 0 & -4.906306 & 0.804314 & -0.71582 \\
\hline 69 & 1 & 0 & -1.604098 & -0.015299 & 1.844567 \\
\hline 70 & 6 & 0 & -2.843819 & 1.205204 & 3.09628 \\
\hline 71 & 6 & 0 & -3.544714 & -0.910985 & 1.91791 \\
\hline 72 & 1 & ) & -4.961632 & 3.516230 & -0.38738 \\
\hline 73 & 1 & 0 & -3.521522 & 3.683363 & 0.64873 \\
\hline 74 & 1 & 0 & -3.355297 & 3.106415 & -1.03322 \\
\hline 75 & 1 & 0 & -4.384786 & 0.866425 & -1.68271 \\
\hline 76 & 1 & 0 & -4.952151 & -0.251655 & -0.42422 \\
\hline 77 & 1 & 0 & -5.929031 & 1.182842 & -0.847996 \\
\hline 78 & 1 & 0 & -3.888645 & 1.525273 & 3.20730 \\
\hline 79 & 1 & 0 & -2.569159 & 0.646334 & 4.00107 \\
\hline 80 & 1 & 0 & -2.210414 & 2.100473 & 3.04487 \\
\hline 81 & 1 & 0 & -4.606215 & -0.629102 & 1.96514 \\
\hline 82 & 1 & 0 & -3.391468 & -1.547517 & 1.03704 \\
\hline 83 & 1 & 0 & -3.314853 & -1.492265 & 2.82097 \\
\hline 84 & 3 & 0 & -1.844636 & -0.175433 & -0.83579 \\
\hline 85 & 8 & 0 & -2.943795 & -1.771530 & -1.39392 \\
\hline 86 & 6 & 0 & -1.998430 & -2.863968 & -1.31118 \\
\hline 87 & 6 & 0 & -1.808487 & -3.339464 & -2.7448 \\
\hline 88 & 6 & 0 & -3.190229 & -3.080651 & -3.34898 \\
\hline 89 & 6 & 0 & -3.570972 & -1.760140 & -2.68765 \\
\hline 90 & 1 & 0 & -1.081162 & -2.451018 & -0.87019 \\
\hline
\end{tabular}




$\begin{array}{llllll}91 & 1 & 0 & -2.437247 & -3.651206 & -0.678018 \\ 92 & 1 & 0 & -1.052457 & -2.715922 & -3.240925 \\ 93 & 1 & 0 & -1.497932 & -4.388723 & -2.800809 \\ 94 & 1 & 0 & -3.185539 & -3.008273 & -4.441916 \\ 95 & 1 & 0 & -3.891492 & -3.872403 & -3.053124 \\ 96 & 1 & 0 & -3.183000 & -0.904254 & -3.261258 \\ 97 & 1 & 0 & -4.651877 & -1.634816 & -2.549267\end{array}$

\section{Thermodynamics:}

Zero-point correction $=$ Thermal correction to Energy= Thermal correction to Enthalpy= Thermal correction to Gibbs Free Energy= Sum of electronic and zero-point Energies= Sum of electronic and thermal Energies= Sum of electronic and thermal Enthalpies= Sum of electronic and thermal Free Energies=
0.881000 (Hartree/Particle)

0.927310

0.928254

0.803911

$-1507.315279$

$-1507.268969$

$-1507.268025$

$-1507.392368$ 
Structure 19c - M06-2x/jul-cc-pVDZ

Input:

$\# \mathrm{~m} 062 \mathrm{x} / \mathrm{jul}-\mathrm{cc}-\mathrm{pvDz}$ opt=(calcfc,noeigen $)$ optcyc $=50$ freq $\mathrm{scrf}=(\mathrm{pcm}$, solve $\mathrm{nt}=\mathrm{THF}$ )

Optimized Structure:

\begin{tabular}{|c|c|c|c|c|}
\hline \multirow{3}{*}{$\begin{array}{l}\text { Center } \\
\text { Number } \\
1\end{array}$} & \multirow{2}{*}{$\begin{array}{l}\text { Atomic } \\
\text { Number }\end{array}$} & \multirow{2}{*}{$\begin{array}{l}\text { Atomic } \\
\text { Type }\end{array}$} & \multicolumn{2}{|c|}{ Coordinates (Angstroms } \\
\hline & & & $\mathrm{X}$ & Z \\
\hline & 7 & 2.276509 & -1.191622 & -0.304594 \\
\hline 2 & 8 & 0.327177 & -0.359447 & 2.145656 \\
\hline 3 & 6 & 3.362543 & -0.206704 & -0.002781 \\
\hline 4 & 6 & 2.216179 & -2.397126 & 0.207215 \\
\hline 5 & 6 & 1.226711 & -0.885484 & -1.174486 \\
\hline 6 & 6 & 0.957394 & -3.052575 & -0.276504 \\
\hline 7 & 6 & 0.690566 & -2.253513 & -1.563741 \\
\hline 8 & 1 & 1.127115 & -0.889905 & 2.219002 \\
\hline 9 & 1 & 2.855671 & -2.717272 & 1.019094 \\
\hline 10 & 1 & 0.170742 & -2.840004 & 0.474934 \\
\hline 11 & 1 & 1.034691 & -4.135789 & -0.409257 \\
\hline 12 & 1 & -0.362648 & -2.263413 & -1.868815 \\
\hline 13 & 1 & 1.2 & -2.693342 & -2.390804 \\
\hline 14 & 3 & -0.2 & -0.051632 & 0.454761 \\
\hline 15 & 1 & 1.505989 & -0.177572 & -1.956584 \\
\hline 16 & 6 & 4.162325 & -0.645986 & 1.220960 \\
\hline 17 & 6 & 4.279524 & -0.155197 & -1.228126 \\
\hline 18 & 6 & 2.725736 & 1.156243 & 0.269068 \\
\hline 19 & 1 & 4.683610 & -1.595700 & 1.052038 \\
\hline 20 & 1 & 919747 & 0.120233 & 1.422228 \\
\hline 21 & 1 & .520 & -0.732792 & 2.106134 \\
\hline 22 & 1 & 4.706504 & -1.145762 & -1.429976 \\
\hline 23 & 1 & 3.736849 & 0.187928 & -2.116272 \\
\hline 24 & 1 & 5.099540 & 0.546335 & -1.030836 \\
\hline 25 & 1 & 1.984091 & 1.069155 & 1.074586 \\
\hline 26 & 1 & 3.518229 & 1.856299 & 0.564067 \\
\hline 27 & 1 & 2.235793 & 1.549678 & -0.627499 \\
\hline 28 & 8 & -0.554795 & 1.834622 & 0.005760 \\
\hline 29 & 6 & -0.338 & 2.524613 & -1.232796 \\
\hline 30 & 6 & -0.096712 & 3.991950 & -0.867373 \\
\hline 31 & 6 & 0.399051 & 3.899166 & 0.579588 \\
\hline 32 & 6 & -0.435299 & 2.742739 & 1.112063 \\
\hline 33 & 1 & 0.529359 & 2.071040 & -1.730988 \\
\hline 34 & 1 & -1.217031 & 2.386541 & -1.876491 \\
\hline 35 & 1 & 0.618645 & 4.472243 & -1.543249 \\
\hline 36 & 1 & -1.038986 & 4.553552 & -0.906266 \\
\hline 37 & 1 & 1.465638 & 3.642241 & 0.610048 \\
\hline 38 & 1 & 0.243530 & 4.823318 & 1.146271 \\
\hline 39 & 1 & 0.016900 & 2.180689 & 1.937498 \\
\hline
\end{tabular}




$\begin{array}{rrrrrr}40 & 1 & 0 & -1.440754 & 3.083065 & 1.408293 \\ 41 & 7 & 0 & -2.084172 & -0.942335 & -0.049741 \\ 42 & 1 & 0 & -1.703503 & -1.870139 & -0.229644 \\ 43 & 6 & 0 & -2.666846 & -0.484215 & -1.327981 \\ 44 & 6 & 0 & -3.001543 & -1.065544 & 1.104608 \\ 45 & 1 & 0 & -1.800716 & -0.261541 & -1.974303 \\ 46 & 6 & 0 & -3.502366 & -1.571592 & -2.007172 \\ 47 & 6 & 0 & -3.472310 & 0.801082 & -1.172595 \\ 48 & 1 & 0 & -4.037862 & -1.212962 & 0.753314 \\ 49 & 6 & 0 & -2.580362 & -2.288715 & 1.912723 \\ 50 & 6 & 0 & -2.937275 & 0.184165 & 1.982661 \\ 51 & 1 & 0 & -2.902415 & -2.476515 & -2.176802 \\ 52 & 1 & 0 & -4.368728 & -1.843419 & -1.387653 \\ 53 & 1 & 0 & -3.876400 & -1.223545 & -2.979299 \\ 54 & 1 & 0 & -2.883303 & 1.575699 & -0.669871 \\ 55 & 1 & 0 & -3.767279 & 1.163204 & -2.165864 \\ 56 & 1 & 0 & -4.390878 & 0.625502 & -0.596180 \\ 57 & 1 & 0 & -2.687171 & -3.208487 & 1.319168 \\ 58 & 1 & 0 & -1.529319 & -2.161875 & 2.211137 \\ 59 & 1 & 0 & -3.197284 & -2.388379 & 2.814500 \\ 60 & 1 & 0 & -1.910963 & 0.299268 & 2.359449 \\ 61 & 1 & 0 & -3.219703 & 1.087885 & 1.432473 \\ 62 & 1 & 0 & -3.622611 & 0.075889 & 2.835171 \\ ------------------------------------------------------\end{array}$

Thermodynamics:

Zero-point correction $=$

Thermal correction to Energy=

Thermal correction to Enthalpy=

Thermal correction to Gibbs Free Energy=

Sum of electronic and zero-point Energies=

Sum of electronic and thermal Energies=

Sum of electronic and thermal Enthalpies=

Sum of electronic and thermal Free Energies=

\author{
0.559471 (Hartree/Particle) \\ 0.589453 \\ 0.590398
}

0.500140

$-975.995035$

$-975.965052$

$-975.964108$

$-976.054366$ 


\section{Structure 20 endo - M06-2x/jul-cc-pVDZ}

Input:

$\#$ m062x/jul-cc-pvDz opt=(calcfc,noeigen,z-matrix $)$ optcyc $=50$ freq $\mathrm{scrf}=(\mathrm{pcm}$,solvent=THF $)$

Optimized Structure:

\begin{tabular}{|c|c|c|c|c|}
\hline \multirow{2}{*}{$\begin{array}{l}\text { Center } \\
\text { Number }\end{array}$} & Atomic & \multirow{2}{*}{$\begin{array}{l}\text { Atomic } \\
\text { Type }\end{array}$} & \multicolumn{2}{|c|}{ Coordinates (Angstroms } \\
\hline & Number & & $\mathrm{X}$ & $\mathrm{Z}$ \\
\hline 1 & 7 & 1.820714 & -0.771867 & -0.444021 \\
\hline 2 & 8 & 0.644608 & -0.946967 & -1.130918 \\
\hline 3 & 6 & 1.672823 & -1.475586 & 0.935332 \\
\hline 4 & 6 & 2.959929 & -1.291820 & -1.291298 \\
\hline 5 & 6 & 2.156591 & 0.700632 & -0.328132 \\
\hline 6 & 6 & 4.209318 & -0.484835 & -0.946530 \\
\hline 7 & 6 & 3.679514 & 0.829654 & -0.322641 \\
\hline 8 & 1 & 2.618254 & -1.077445 & -2.307214 \\
\hline 9 & 1 & 3.050313 & -2.371874 & -1.176066 \\
\hline 10 & 1 & 4.786748 & -0.296886 & $5-1.857111$ \\
\hline 11 & 1 & 4.866984 & -1.020195 & -0.254822 \\
\hline 12 & 1 & 3.973250 & 1.701920 & -0.915367 \\
\hline 13 & 1 & 4.076825 & 0.974756 & 0.686072 \\
\hline 14 & 1 & 1.720175 & 1.122378 & -1.237937 \\
\hline 15 & 1 & 1.624828 & 1.138385 & 0.518707 \\
\hline 16 & 6 & 1.646542 & -2.986821 & 0.714746 \\
\hline 17 & 6 & 2.803421 & -1.103338 & 1.892520 \\
\hline 18 & 6 & 0.333559 & -1.038620 & 1.531967 \\
\hline 19 & 1 & 2.647784 & -3.408908 & 0.568884 \\
\hline 20 & 1 & 1.220356 & -3.452645 & 51.611506 \\
\hline 21 & 1 & 1.008941 & -3.234736 & $5-0.141385$ \\
\hline 22 & 1 & 3.794286 & -1.329889 & 1.486724 \\
\hline 23 & 1 & 2.758685 & -0.049922 & 2.191874 \\
\hline 24 & 1 & 2.675851 & -1.709753 & 2.797494 \\
\hline 25 & 1 & 0.170487 & 0.042729 & 1.448676 \\
\hline 26 & 1 & -0.496755 & -1.556846 & $6 \quad 1.038632$ \\
\hline 27 & 1 & 0.328123 & -1.309410 & 2.595086 \\
\hline 28 & 3 & -0.851872 & 0.104686 & $5-0.645081$ \\
\hline 29 & 7 & -0.953221 & 1.887039 & -0.024370 \\
\hline 30 & 6 & -1.301467 & 2.613752 & 1.173641 \\
\hline 31 & 6 & -0.714054 & 2.725293 & -1.184295 \\
\hline 32 & 1 & -1.745805 & 3.605826 & $5 \quad 0.940112$ \\
\hline 33 & 6 & -2.358366 & 1.846599 & 1.976366 \\
\hline 34 & 6 & -0.101976 & 2.895822 & 2.109542 \\
\hline 35 & 1 & -0.324150 & 2.056083 & -1.980465 \\
\hline 36 & 6 & -1.995297 & 3.357955 & $5-1.770415$ \\
\hline 37 & 6 & 0.347198 & 3.833051 & -1.015484 \\
\hline 38 & 1 & -2.603683 & 2.348997 & 2.924909 \\
\hline
\end{tabular}




$\begin{array}{rrrrrr}39 & 1 & 0 & -3.277857 & 1.729775 & 1.386823 \\ 40 & 1 & 0 & -1.976254 & 0.840449 & 2.212336 \\ 41 & 1 & 0 & -0.394437 & 3.514416 & 2.974097 \\ 42 & 1 & 0 & 0.301577 & 1.946524 & 2.497311 \\ 43 & 1 & 0 & 0.704936 & 3.410626 & 1.575139 \\ 44 & 1 & 0 & -1.787686 & 3.940796 & -2.682005 \\ 45 & 1 & 0 & -2.718756 & 2.568990 & -2.015400 \\ 46 & 1 & 0 & -2.463518 & 4.034722 & -1.040018 \\ 47 & 1 & 0 & 1.296809 & 3.416280 & -0.652112 \\ 48 & 1 & 0 & 0.535615 & 4.347716 & -1.969866 \\ 49 & 1 & 0 & 0.009120 & 4.588281 & -0.290232 \\ 50 & 8 & 0 & -2.294556 & -1.212595 & -0.833361 \\ 51 & 6 & 0 & -3.411319 & -1.225298 & 0.077900 \\ 52 & 6 & 0 & -3.928174 & -2.663069 & 0.089360 \\ 53 & 6 & 0 & -2.669171 & -3.460156 & -0.262682 \\ 54 & 6 & 0 & -2.017977 & -2.548531 & -1.292481 \\ 55 & 1 & 0 & -3.048242 & -0.917170 & 1.068171 \\ 56 & 1 & 0 & -4.153108 & -0.495744 & -0.268214 \\ 57 & 1 & 0 & -4.362538 & -2.935446 & 1.056902 \\ 58 & 1 & 0 & -4.691562 & -2.803528 & -0.686842 \\ 59 & 1 & 0 & -2.019691 & -3.566222 & 0.617563 \\ 60 & 1 & 0 & -2.884406 & -4.457053 & -0.661997 \\ 61 & 1 & 0 & -0.929934 & -2.642982 & -1.371996 \\ 62 & 1 & 0 & -2.475386 & -2.684871 & -2.284255 \\ --------------------------------------------\end{array}$

\section{Thermodynamics:}

Zero-point correction $=$ Thermal correction to Energy= Thermal correction to Enthalpy= Thermal correction to Gibbs Free Energy= Sum of electronic and zero-point Energies $=$ Sum of electronic and thermal Energies= Sum of electronic and thermal Enthalpies= Sum of electronic and thermal Free Energies=
0.562702 (Hartree/Particle) 0.591188 0.592132

0.503745 $-975.947268$ $-975.918782$ $-975.917837$ $-976.006225$ 
Structure $20_{\text {exo }}-$ M06-2x/jul-cc-pVDZ

Input:

$\# \mathrm{~m} 062 \mathrm{x} / \mathrm{jul}-\mathrm{cc}-\mathrm{pvDz}$ opt=(calcfc,noeigen,z-matrix $)$ optcyc $=50$ freq scrf $=(\mathrm{pcm}$,solvent=THF $)$

Optimized Structure:

\begin{tabular}{|c|c|c|c|c|}
\hline \multirow{2}{*}{$\begin{array}{l}\text { Center } \\
\text { Number }\end{array}$} & Atomic & \multirow{2}{*}{$\begin{array}{l}\text { Atomic } \\
\text { Type }\end{array}$} & \multicolumn{2}{|c|}{ Coordinates (Angstroms } \\
\hline & Number & & $\mathrm{X}$ & $\mathrm{Z}$ \\
\hline 1 & 7 & -1.568712 & -1.392399 & -0.138379 \\
\hline 2 & 8 & -1.183273 & -0.381956 & -0.988898 \\
\hline 3 & 6 & -2.124832 & -0.787581 & 1.167419 \\
\hline 4 & 6 & -2.559950 & -2.247226 & -0.876093 \\
\hline 5 & 6 & -0.408268 & -2.333663 & 0.060940 \\
\hline 6 & 6 & -1.707731 & -3.037810 & -1.874169 \\
\hline 7 & 6 & -0.270051 & -3.001925 & -1.303186 \\
\hline 8 & 1 & -3.288252 & -1.580168 & -1.339189 \\
\hline 9 & 1 & -3.056687 & -2.905142 & -0.155227 \\
\hline 10 & 1 & -1.744940 & -2.575316 & $6-2.864099$ \\
\hline 11 & 1 & -2.088597 & -4.060253 & $3-1.965271$ \\
\hline 12 & 1 & 0.388988 & -2.411837 & $7-1.946351$ \\
\hline 13 & 1 & 0.16 & -4.002181 & -1.195447 \\
\hline 14 & 1 & 0.466226 & -1.742730 & 0.363886 \\
\hline 15 & 1 & -0.690365 & -3.066416 & $\begin{array}{ll}5 & 0.823390\end{array}$ \\
\hline 16 & 6 & -3.470829 & -0.140182 & 20.850250 \\
\hline 17 & 6 & -2.287213 & -1.851990 & 2.253823 \\
\hline 18 & 6 & -1.141373 & 0.279526 & $5 \quad 1.643287$ \\
\hline 19 & 1 & -4.264129 & -0.884286 & $6 \quad 0.710641$ \\
\hline 20 & 1 & -3.752991 & 0.499025 & 1.695554 \\
\hline 21 & 1 & -3.389412 & 0.483990 & -0.046855 \\
\hline 22 & 1 & -2.831339 & -2.738469 & 1.907128 \\
\hline 23 & 1 & -1.321214 & -2.162656 & 2.667348 \\
\hline 24 & 1 & -2.870777 & -1.407434 & 3.069034 \\
\hline 25 & 1 & -0.106839 & -0.085265 & 1.632665 \\
\hline 26 & 1 & -1.208077 & 1.178203 & 1.019557 \\
\hline 27 & 1 & -1.402 & 0.552856 & 2.673236 \\
\hline 28 & 3 & 0.404497 & 0.571609 & -0.603029 \\
\hline 29 & 7 & 2.063502 & -0.027368 & 0.070794 \\
\hline 30 & 6 & 2.726941 & -0.612024 & -1.081228 \\
\hline 31 & 6 & 2.870016 & 0.001790 & 1.269132 \\
\hline 32 & 1 & 1.961627 & -0.679436 & -1.883117 \\
\hline 33 & 6 & 3.856041 & 0.265002 & -1.663190 \\
\hline 34 & 6 & 3.260116 & -2.047497 & -0.893794 \\
\hline 35 & 1 & 3.954752 & -0.046431 & 1.033905 \\
\hline 36 & 6 & 2.654617 & 1.314220 & 2.031645 \\
\hline 37 & 6 & 2.592836 & -1.171963 & 2.236725 \\
\hline 38 & 1 & 4.293625 & -0.178200 & -2.571776 \\
\hline 39 & 1 & 3.459846 & 1.258199 & -1.912275 \\
\hline 40 & 1 & 4.666495 & 0.394402 & -0.930577 \\
\hline
\end{tabular}




$\begin{array}{rrrrrr}41 & 1 & 0 & 3.647024 & -2.453626 & -1.840832 \\ 42 & 1 & 0 & 4.082397 & -2.063190 & -0.162910 \\ 43 & 1 & 0 & 2.467321 & -2.714376 & -0.528064 \\ 44 & 1 & 0 & 3.221259 & 1.343174 & 2.975205 \\ 45 & 1 & 0 & 2.954227 & 2.168027 & 1.409351 \\ 46 & 1 & 0 & 1.585625 & 1.428703 & 2.271957 \\ 47 & 1 & 0 & 2.715755 & -2.137499 & 1.731349 \\ 48 & 1 & 0 & 3.265020 & -1.149856 & 3.110111 \\ 49 & 1 & 0 & 1.556728 & -1.115934 & 2.607269 \\ 50 & 8 & 0 & -0.127063 & 2.417682 & -0.966726 \\ 51 & 6 & 0 & 0.347919 & 3.481530 & -0.117740 \\ 52 & 6 & 0 & -0.723737 & 4.569398 & -0.168827 \\ 53 & 6 & 0 & -1.989460 & 3.762166 & -0.470500 \\ 54 & 6 & 0 & -1.453639 & 2.716969 & -1.438421 \\ 55 & 1 & 0 & 0.472369 & 3.079490 & 0.897568 \\ 56 & 1 & 0 & 1.325690 & 3.812491 & -0.487184 \\ 57 & 1 & 0 & -0.779104 & 5.135667 & 0.766719 \\ 58 & 1 & 0 & -0.520271 & 5.270573 & -0.988474 \\ 59 & 1 & 0 & -2.367214 & 3.280331 & 0.442212 \\ 60 & 1 & 0 & -2.793791 & 4.363780 & -0.907105 \\ 61 & 1 & 0 & -2.009852 & 1.773528 & -1.453912 \\ 62 & 1 & 0 & -1.384013 & 3.123566 & -2.458695 \\ --------------------------------------------------------\end{array}$

\section{Thermodynamics:}

Zero-point correction $=$

Thermal correction to Energy=

Thermal correction to Enthalpy=

Thermal correction to Gibbs Free Energy=

Sum of electronic and zero-point Energies=

Sum of electronic and thermal Energies=

Sum of electronic and thermal Enthalpies=

Sum of electronic and thermal Free Energies=

\subsection{5 (Hartree/Particle)}

0.591287

0.592231

0.504516

$-975.956177$

$-975.927746$

$-975.926801$

$-976.014516$ 
Structure $20_{\text {endo-TS }}$ - M06-2x/jul-cc-pVDZ

Input:

$\#$ m062x/jul-cc-pvDz opt=(calcfc,noeigen,z-matrix,ts) optcyc=50 freq scr $\mathrm{f}=(\mathrm{pcm}$, solvent $=\mathrm{THF})$

Optimized Structure:

\begin{tabular}{|c|c|c|c|c|}
\hline \multirow{2}{*}{$\begin{array}{l}\text { Center } \\
\text { Number }\end{array}$} & Atomic & \multirow{2}{*}{$\begin{array}{l}\text { Atomic } \\
\text { Type }\end{array}$} & \multicolumn{2}{|c|}{ Coordinates (Angstroms } \\
\hline & & & $\mathrm{X}$ & Z \\
\hline 1 & 7 & 1.432891 & -1.294652 & -0.512235 \\
\hline 2 & 8 & 0.252509 & -1.265775 & -1.235685 \\
\hline 3 & 6 & 1.103764 & -1.927387 & 0.862040 \\
\hline 4 & 6 & 2.490200 & -2.081241 & -1.260173 \\
\hline 5 & 6 & 2.013133 & 0.117570 & -0.471972 \\
\hline 6 & 6 & 3.848404 & -1.432633 & -0.957667 \\
\hline 7 & 6 & 3.516001 & -0.066343 & -0.292304 \\
\hline 8 & 1 & 2.200839 & -1.949877 & -2.305206 \\
\hline 9 & 1 & 2.433844 & -3.143554 & -1.014856 \\
\hline 10 & 1 & 4.400197 & -1.291824 & -1.893861 \\
\hline 11 & 1 & 4.468572 & -2.066466 & -0.312931 \\
\hline 12 & 1 & 4.040944 & 0.755023 & -0.795981 \\
\hline 13 & 1 & 3.848967 & -0.054111 & 0.751968 \\
\hline 14 & 1 & 1.888394 & 0.373742 & -1.539331 \\
\hline 15 & 1 & 1.078180 & 1.070004 & 0.007933 \\
\hline 16 & 6 & 0.652110 & -3.375181 & 0.650599 \\
\hline 17 & 6 & 2.296961 & -1.890029 & 1.813401 \\
\hline 18 & 6 & -0.048051 & -1.143510 & 1.485442 \\
\hline 19 & 1 & 1.493429 & -4.065624 & 0.519789 \\
\hline 20 & 1 & 0.104206 & -3.695572 & 1.545592 \\
\hline 21 & 1 & -0.018336 & -3.440845 & -0.212723 \\
\hline 22 & 1 & 3.194594 & -2.345300 & 1.381156 \\
\hline 23 & 1 & 2.520402 & -0.867561 & 2.134301 \\
\hline 24 & 1 & 2.027872 & -2.474001 & 2.702776 \\
\hline 25 & 1 & 0.154029 & -0.069659 & 1.475227 \\
\hline 26 & 1 & -0.992058 & -1.350223 & 0.966873 \\
\hline 27 & 1 & -0.158483 & -1.463073 & 2.529422 \\
\hline 28 & 3 & -0.793305 & 0.217738 & -0.707192 \\
\hline 29 & 7 & 0.027471 & 1.884778 & 0.021984 \\
\hline 30 & 6 & -0.067956 & 2.687512 & 1.241401 \\
\hline 31 & 6 & 0.195783 & 2.679467 & -1.197360 \\
\hline 32 & 1 & -0.117794 & 3.759683 & 0.983888 \\
\hline 33 & 6 & -1.345089 & 2.378393 & 2.027464 \\
\hline 34 & 6 & 1.167283 & 2.517127 & 2.139846 \\
\hline 35 & 1 & 0.322733 & 1.946419 & -2.016589 \\
\hline 36 & 6 & -1.041921 & 3.514154 & -1.544525 \\
\hline 37 & 6 & 1.463612 & 3.548447 & -1.206995 \\
\hline 38 & 1 & -1.419087 & 2.998410 & 2.933358 \\
\hline 39 & 1 & -2.227606 & 2.564867 & 1.400900 \\
\hline
\end{tabular}




$\begin{array}{rrrrrr}40 & 1 & 0 & -1.361716 & 1.322581 & 2.336410 \\ 41 & 1 & 0 & 1.098608 & 3.156011 & 3.032105 \\ 42 & 1 & 0 & 1.267824 & 1.474477 & 2.476094 \\ 43 & 1 & 0 & 2.081435 & 2.778854 & 1.590512 \\ 44 & 1 & 0 & -0.909530 & 4.041325 & -2.500481 \\ 45 & 1 & 0 & -1.926934 & 2.867610 & -1.620421 \\ 46 & 1 & 0 & -1.238599 & 4.270219 & -0.770722 \\ 47 & 1 & 0 & 2.349545 & 2.928253 & -1.014083 \\ 48 & 1 & 0 & 1.591377 & 4.047115 & -2.178397 \\ 49 & 1 & 0 & 1.416472 & 4.328965 & -0.434259 \\ 50 & 8 & 0 & -2.627606 & -0.351123 & -0.785872 \\ 51 & 6 & 0 & -3.603781 & 0.009677 & 0.211767 \\ 52 & 6 & 0 & -4.640753 & -1.108681 & 0.196604 \\ 53 & 6 & 0 & -3.795202 & -2.316259 & -0.216015 \\ 54 & 6 & 0 & -2.865008 & -1.698094 & -1.250630 \\ 55 & 1 & 0 & -3.097345 & 0.074993 & 1.185599 \\ 56 & 1 & 0 & -4.011789 & 0.994335 & -0.044739 \\ 57 & 1 & 0 & -5.130098 & -1.229004 & 1.168618 \\ 58 & 1 & 0 & -5.409945 & -0.908139 & -0.560435 \\ 59 & 1 & 0 & -3.219057 & -2.693286 & 0.640222 \\ 60 & 1 & 0 & -4.387310 & -3.139582 & -0.629131 \\ 61 & 1 & 0 & -1.888634 & -2.187238 & -1.340743 \\ 62 & 1 & 0 & -3.346918 & -1.644792 & -2.237608 \\ ----------------------------------------------------------\end{array}$

Thermodynamics:

$\begin{array}{lc}\text { Zero-point correction= } & 0.557955 \text { (Hartree/Particle) } \\ \text { Thermal correction to Energy= } & 0.586077 \\ \text { Thermal correction to Enthalpy= } & 0.587022 \\ \text { Thermal correction to Gibbs Free Energy }= & 0.499585 \\ \text { Sum of electronic and zero-point Energies }= & -975.926542 \\ \text { Sum of electronic and thermal Energies }= & -975.898420 \\ \text { Sum of electronic and thermal Enthalpies }= & -975.897476 \\ \text { Sum of electronic and thermal Free Energies }= & -975.984912\end{array}$ 
Structure 20 exo-Ts $_{\text {- M06-2x/jul-cc-pVDZ }}$

Input:

\#m062x/jul-cc-pvDz opt=(calcfc,noeigen,z-matrix,ts $)$ optcyc=50 freq scr
$\mathrm{f}=(\mathrm{pcm}$, solvent=THF $)$

Optimized Structure:

\begin{tabular}{|c|c|c|c|c|}
\hline \multirow{2}{*}{$\begin{array}{l}\text { Center } \\
\text { Number }\end{array}$} & Atomic & \multirow{2}{*}{$\begin{array}{l}\text { Atomic } \\
\text { Type }\end{array}$} & \multicolumn{2}{|c|}{ Coordinates (Angstroms } \\
\hline & & & $\mathrm{X}$ & $\mathrm{Z}$ \\
\hline 1 & 7 & 0.986391 & -1.758684 & -0.202362 \\
\hline 2 & 8 & 0.031029 & -1.252584 & -1.073065 \\
\hline 3 & 6 & 0.285617 & -2.229084 & 1.083879 \\
\hline 4 & 6 & 1.671208 & -2.890468 & -0.919560 \\
\hline 5 & 6 & 2.064524 & -0.709626 & 0.018887 \\
\hline 6 & 6 & 2.650936 & -2.209776 & -1.873567 \\
\hline 7 & 6 & 2.836606 & -0.779139 & -1.303339 \\
\hline 8 & 1 & 0.905857 & -3.490037 & -1.418026 \\
\hline 9 & 1 & 2.194262 & -3.499149 & -0.174996 \\
\hline 10 & 1 & 2.247133 & -2.184143 & $3-2.891192$ \\
\hline 11 & 1 & 3.593917 & -2.767798 & $8-1.904583$ \\
\hline 12 & 1 & 2.460969 & -0.048553 & $3-2.030785$ \\
\hline 13 & 1 & 3.894847 & -0.543134 & $4-1.131504$ \\
\hline 14 & 1 & 1.440915 & 0.613886 & $5 \quad 0.183130$ \\
\hline 15 & 1 & 2.711671 & -1.114855 & $5 \quad 0.810334$ \\
\hline 16 & 6 & -0.571407 & -3.450754 & $4 \quad 0.752610$ \\
\hline 17 & 6 & 1.289652 & -2.572706 & $6 \quad 2.186445$ \\
\hline 18 & 6 & -0.617663 & -1.100191 & $1 \quad 1.573685$ \\
\hline 19 & 1 & 0.034020 & -4.354511 & 10.614180 \\
\hline 20 & 1 & -1.258232 & -3.630733 & $3 \quad 1.589120$ \\
\hline 21 & 1 & -1.161336 & -3.263194 & $4-0.151567$ \\
\hline 22 & 1 & 2.081145 & -3.250549 & $9 \quad 1.847296$ \\
\hline 23 & 1 & 1.749727 & -1.672106 & $\begin{array}{ll}6 & 2.606718\end{array}$ \\
\hline 24 & 1 & 0.741356 & -3.080212 & $2 \quad 2.990336$ \\
\hline 25 & 1 & -0.072731 & -0.151152 & $2 \quad 1.604345$ \\
\hline 26 & 1 & -1.501418 & -0.997069 & $9 \quad 0.933735$ \\
\hline 27 & 1 & -0.955530 & -1.338915 & $\begin{array}{ll}5 & 2.590070\end{array}$ \\
\hline 28 & 3 & -0.547881 & 0.460394 & $\begin{array}{ll}4 & -0.657509\end{array}$ \\
\hline 29 & 7 & 0.776 & 1.716540 & $\begin{array}{l}0.107384 \\
\end{array}$ \\
\hline 30 & 6 & 263993 & 2.420336 & $\begin{array}{ll}6 & -1.083641\end{array}$ \\
\hline 31 & 6 & 0.861700 & 2.510700 & ) 1.334996 \\
\hline 32 & 1 & 1.170605 & 1.700166 & $6 \quad-1.916168$ \\
\hline 33 & 6 & 0.401937 & 3.632001 & $1-1.456308$ \\
\hline 34 & 6 & 2.748756 & 2.808116 & $6-1.011303$ \\
\hline 35 & 1 & 1.258351 & 3.514089 & $\begin{array}{l}9 \\
9\end{array}$ \\
\hline 36 & 6 & -0.514024 & 2.730704 & $\begin{array}{ll}4 & 1.971458\end{array}$ \\
\hline 37 & 6 & 1.830215 & 1.892195 & $5 \quad 2.352846$ \\
\hline 38 & 1 & 0.754080 & 4.093017 & $\begin{array}{ll}7 & -2.390355\end{array}$ \\
\hline 39 & 1 & -0.645498 & 3.328427 & $\begin{array}{ll}7 & -1.589599\end{array}$ \\
\hline 40 & 1 & 0.436338 & 4.400813 & $3-0.671152$ \\
\hline
\end{tabular}




$\begin{array}{rrrrrr}41 & 1 & 0 & 3.092330 & 3.226612 & -1.968285 \\ 42 & 1 & 0 & 2.923865 & 3.566000 & -0.234373 \\ 43 & 1 & 0 & 3.359381 & 1.927005 & -0.775919 \\ 44 & 1 & 0 & -0.444366 & 3.364999 & 2.867863 \\ 45 & 1 & 0 & -1.190998 & 3.212165 & 1.252925 \\ 46 & 1 & 0 & -0.959495 & 1.770005 & 2.269976 \\ 47 & 1 & 0 & 2.833541 & 1.794352 & 1.917770 \\ 48 & 1 & 0 & 1.897091 & 2.514877 & 3.256309 \\ 49 & 1 & 0 & 1.499303 & 0.888031 & 2.654464 \\ 50 & 8 & 0 & -2.460424 & 0.528057 & -0.898447 \\ 51 & 6 & 0 & -3.348453 & 1.190198 & 0.023448 \\ 52 & 6 & 0 & -4.684083 & 0.464236 & -0.098264 \\ 53 & 6 & 0 & -4.238309 & -0.955765 & -0.457367 \\ 54 & 6 & 0 & -3.071926 & -0.682065 & -1.397338 \\ 55 & 1 & 0 & -2.931830 & 1.096212 & 1.036948 \\ 56 & 1 & 0 & -3.395967 & 2.251712 & -0.245985 \\ 57 & 1 & 0 & -5.267571 & 0.518488 & 0.826582 \\ 58 & 1 & 0 & -5.280194 & 0.892683 & -0.914397 \\ 59 & 1 & 0 & -3.891915 & -1.487959 & 0.439286 \\ 60 & 1 & 0 & -5.024907 & -1.549727 & -0.934353 \\ 61 & 1 & 0 & -2.296891 & -1.457246 & -1.405854 \\ 62 & 1 & 0 & -3.422261 & -0.495910 & -2.422787 \\ ---------------------------------------------------------\end{array}$

\section{Thermodynamics:}

Zero-point correction $=$

Thermal correction to Energy=

Thermal correction to Enthalpy=

Thermal correction to Gibbs Free Energy=

Sum of electronic and zero-point Energies=

Sum of electronic and thermal Energies=

Sum of electronic and thermal Enthalpies=

Sum of electronic and thermal Free Energies=

\subsection{8 (Hartree/Particle)} 0.586108

0.587052

0.501018

$-975.938235$

$-975.910195$

$-975.909251$

$-975.995285$ 


\section{Structure 20' ${ }^{\prime}$ endo (no explicit THF) - M06-2x/jul-cc-pVDZ}

Input:

$\#$ 062x/jul-cc-pvDz opt=(calcfc,noeigen,z-matrix $)$ optcyc $=50$ freq $\mathrm{scrf}=(\mathrm{pcm}$,solvent=THF $)$

Optimized Structure:

\begin{tabular}{|c|c|c|c|c|}
\hline \multirow{3}{*}{$\begin{array}{c}\text { Center } \\
\text { Number } \\
1\end{array}$} & \multirow{2}{*}{$\begin{array}{l}\text { Atomic } \\
\text { Number }\end{array}$} & \multirow{2}{*}{$\begin{array}{l}\text { Atomic } \\
\text { Type }\end{array}$} & \multicolumn{2}{|c|}{ Coordinates (Angstroms } \\
\hline & & & $\mathrm{X}$ & $\mathrm{Z}$ \\
\hline & 7 & 0.000000 & 0.000000 & 0.000000 \\
\hline 2 & 8 & 0.000000 & 0.000000 & 1.374539 \\
\hline 3 & 6 & 1.478636 & 0.000000 & -0.482144 \\
\hline 4 & 6 & -0.813207 & -1.181318 & -0.479826 \\
\hline 5 & 6 & -0.796754 & 1.173624 & -0.534333 \\
\hline 6 & 6 & -1.435519 & -0.810012 & -1.823369 \\
\hline 7 & 6 & -1.437161 & 0.738054 & -1.852331 \\
\hline 8 & 1 & -1.576549 & -1.276617 & 0.296620 \\
\hline 9 & 1 & -0.200723 & -2.082747 & -0.476987 \\
\hline 10 & 1 & -2.449898 & -1.216830 & -1.881619 \\
\hline 11 & 1 & -0.874598 & -1.225948 & -2.665815 \\
\hline 12 & 1 & -2.454772 & 1.137177 & -1.911296 \\
\hline 13 & 1 & -0.889579 & 1.116152 & -2.720292 \\
\hline 14 & 1 & -1.549867 & 1.336806 & 0.241962 \\
\hline 15 & 1 & -0.167897 & 2.065514 & -0.572451 \\
\hline 16 & 6 & 2.100760 & -1.357100 & -0.160240 \\
\hline 17 & 6 & 1.591705 & 0.286472 & -1.978446 \\
\hline 18 & 6 & 2.215776 & 1.085649 & 0.303822 \\
\hline 19 & 1 & 1.824069 & -2.128837 & -0.888291 \\
\hline 20 & 1 & 3.191210 & -1.246557 & -0.196092 \\
\hline 21 & 1 & 1.817548 & -1.676257 & 0.848707 \\
\hline 22 & 1 & 1.026052 & -0.423256 & -2.590026 \\
\hline 23 & 1 & 1.290995 & 1.310530 & -2.227231 \\
\hline 24 & 1 & 2.648925 & 0.178122 & -2.249368 \\
\hline 25 & 1 & 1.649294 & 2.022973 & 0.357355 \\
\hline 26 & 1 & 2.434237 & 0.745370 & 1.321227 \\
\hline 27 & 1 & 3.167873 & 1.288569 & -0.201698 \\
\hline 28 & 3 & 0.224630 & 1.629046 & 2.257933 \\
\hline 29 & -1 & 0.370359 & 1.696841 & -0.318759 \\
\hline 30 & 7 & -0.156987 & 3.428498 & 1.916828 \\
\hline 31 & 6 & 0.607968 & 4.623242 & 1.640978 \\
\hline 32 & 6 & -1.589305 & 3.641057 & 2.027118 \\
\hline 33 & 1 & 0.085131 & 5.536815 & 1.997273 \\
\hline 34 & 6 & 1.948548 & 4.571971 & 2.382610 \\
\hline 35 & 6 & 0.898806 & 4.858331 & 0.140405 \\
\hline 36 & 1 & -2.047838 & 2.634024 & 2.117269 \\
\hline 37 & 6 & -2.015935 & 4.403077 & 3.300608 \\
\hline 38 & 6 & -2.270180 & 4.301082 & 0.810311 \\
\hline 39 & 1 & 2.592613 & 5.430230 & 2.137105 \\
\hline 40 & 1 & 1.785697 & 4.550338 & 3.468300 \\
\hline
\end{tabular}




$\begin{array}{rrrrrr}41 & 1 & 0 & 2.485398 & 3.651668 & 2.101138 \\ 42 & 1 & 0 & 1.406727 & 5.822203 & -0.027332 \\ 43 & 1 & 0 & 1.555635 & 4.062447 & -0.244337 \\ 44 & 1 & 0 & -0.023706 & 4.848609 & -0.451119 \\ 45 & 1 & 0 & -3.111247 & 4.492547 & 3.374298 \\ 46 & 1 & 0 & -1.647358 & 3.879988 & 4.193051 \\ 47 & 1 & 0 & -1.597722 & 5.420533 & 3.305143 \\ 48 & 1 & 0 & -2.029652 & 3.759603 & -0.115265 \\ 49 & 1 & 0 & -3.363865 & 4.316065 & 0.931252 \\ 50 & 1 & 0 & -1.932927 & 5.341766 & 0.692280\end{array}$

Thermodynamics:

Zero-point correction $=$

Thermal correction to Energy=

Thermal correction to Enthalpy=

0.444352 (Hartree/Particle)

0.466340

0.467284

Thermal correction to Gibbs Free Energy=

Sum of electronic and zero-point Energies=

Sum of electronic and thermal Energies=

0.394744

Sum of electronic and thermal Enthalpies=

$-743.676814$

$-743.654826$

$-743.653881$

Sum of electronic and thermal Free Energies=

$-743.726422$ 


\section{Structure 20' ${ }_{\text {exo }}$ (no explicit THF) - M06-2x/jul-cc-pVDZ}

Input:

$\#$ 062x/jul-cc-pvDz opt=(calcfc,noeigen,z-matrix $)$ optcyc $=50$ freq $\mathrm{scrf}=(\mathrm{pcm}$,solvent=THF $)$

Optimized Structure:

\begin{tabular}{|c|c|c|c|c|}
\hline \multirow{3}{*}{$\begin{array}{l}\text { Center } \\
\text { Number } \\
1\end{array}$} & \multirow{2}{*}{$\begin{array}{l}\text { Atomic } \\
\text { Number }\end{array}$} & \multirow{2}{*}{$\begin{array}{r}\text { Atomic } \\
\text { Type }\end{array}$} & \multicolumn{2}{|c|}{ Coordinates (Angstrom } \\
\hline & & & $\mathrm{X}$ & $\mathrm{Z}$ \\
\hline & 7 & 0.000000 & 0.000000 & 0.000000 \\
\hline 2 & 8 & 0.000000 & 0.000000 & 1.375778 \\
\hline 3 & 6 & 1.448727 & 0.000000 & -0.529612 \\
\hline 4 & 6 & -0.804114 & -1.189733 & -0.443454 \\
\hline 5 & 6 & -0.835383 & 1.157698 & -0.486540 \\
\hline 6 & 6 & -2.255114 & -0.793118 & -0.153868 \\
\hline 7 & 6 & -2.252677 & 0.753114 & -0.093732 \\
\hline 8 & 1 & -0.453868 & -2.052065 & 0.125155 \\
\hline 9 & 1 & -0.631462 & -1.347480 & -1.513249 \\
\hline 10 & 1 & -2.588279 & -1.218799 & 0.796560 \\
\hline 11 & 1 & -2.912353 & -1.173247 & -0.942622 \\
\hline 12 & 1 & -2.479176 & 1.104703 & 0.916792 \\
\hline 13 & 1 & -2.978793 & 1.199672 & -0.781134 \\
\hline 14 & 1 & -0.480812 & 2.073372 & 0.003056 \\
\hline 15 & 1 & -0.736747 & 1.215685 & -1.574514 \\
\hline 16 & 6 & 2.056416 & -1.370317 & -0.241000 \\
\hline 17 & 6 & 1.497255 & 0.299258 & -2.029167 \\
\hline 18 & 6 & 2.220030 & 1.077726 & 0.228924 \\
\hline 19 & 1 & 1.674455 & -2.140392 & -0.921878 \\
\hline 20 & 1 & 3.141649 & -1.302549 & -0.383215 \\
\hline 21 & 1 & 1.858147 & -1.663197 & 0.795713 \\
\hline 22 & 1 & 0.811436 & -0.325331 & -2.613574 \\
\hline 23 & 1 & 1.292704 & 1.354452 & -2.242473 \\
\hline 24 & 1 & 2.514317 & 0.081044 & -2.376439 \\
\hline 25 & 1 & 1.669849 & 2.026121 & 0.265192 \\
\hline 26 & 1 & 2.442892 & 0.750100 & 1.249283 \\
\hline 27 & 1 & 3.170896 & 1.252129 & -0.289053 \\
\hline 28 & 3 & 0.372110 & 1.577888 & 2.295156 \\
\hline 29 & -1 & 0.003852 & 1.702421 & 0.345833 \\
\hline 30 & 7 & -0.100365 & 3.372216 & 2.056401 \\
\hline 31 & 6 & -1.490319 & 3.555106 & 2.440004 \\
\hline 32 & 6 & 0.573980 & 4.590814 & 1.665753 \\
\hline 33 & 1 & -1.902730 & 2.541028 & 2.619687 \\
\hline 34 & 6 & -1.681283 & 4.317615 & 3.768522 \\
\hline 35 & 6 & -2.398693 & 4.190292 & 1.367851 \\
\hline 36 & 1 & 0.074327 & 5.487285 & 2.089269 \\
\hline 37 & 6 & 2.009494 & 4.601501 & 2.204554 \\
\hline 38 & 6 & 0.623980 & 4.815296 & 0.137924 \\
\hline 39 & 1 & -2.743633 & 4.379289 & 4.052343 \\
\hline 40 & 1 & -1.133553 & 3.810064 & 4.573830 \\
\hline
\end{tabular}




$\begin{array}{rrrrrr}41 & 1 & 0 & -1.297754 & 5.345564 & 3.690723 \\ 42 & 1 & 0 & -3.451420 & 4.188662 & 1.689066 \\ 43 & 1 & 0 & -2.108290 & 5.234941 & 1.180236 \\ 44 & 1 & 0 & -2.323438 & 3.640349 & 0.419348 \\ 45 & 1 & 0 & 2.574694 & 5.482899 & 1.864466 \\ 46 & 1 & 0 & 2.004266 & 4.586611 & 3.302887 \\ 47 & 1 & 0 & 2.539261 & 3.700958 & 1.854712 \\ 48 & 1 & 0 & -0.381168 & 4.781049 & -0.299143 \\ 49 & 1 & 0 & 1.079699 & 5.786098 & -0.116207 \\ 50 & 1 & 0 & 1.226032 & 4.026372 & -0.339864\end{array}$

Thermodynamics:

$\begin{array}{lc}\text { Zero-point correction= } & 0.443714 \text { (Hartree/Particle) } \\ \text { Thermal correction to Energy= } & 0.466010 \\ \text { Thermal correction to Enthalpy= } & 0.466954 \\ \text { Thermal correction to Gibbs Free Energy }= & 0.393955 \\ \text { Sum of electronic and zero-point Energies }= & -743.685850 \\ \text { Sum of electronic and thermal Energies }= & -743.663555 \\ \text { Sum of electronic and thermal Enthalpies }= & -743.662611 \\ \text { Sum of electronic and thermal Free Energies }= & -743.735610\end{array}$


Structure 21 endo - M06-2x/jul-cc-pVDZ

Input:

$\# \mathrm{~m} 062 \mathrm{x} / \mathrm{jul}-\mathrm{cc}-\mathrm{pvDz}$ opt$=($ calcfc,noeigen, $\mathrm{z}-\mathrm{matrix})$ optcyc $=50$ freq $\mathrm{scrf}=(\mathrm{pcm}, \mathrm{solvent}=\mathrm{THF})$

Optimized Structure:

\begin{tabular}{|c|c|c|c|c|}
\hline \multirow{2}{*}{$\begin{array}{l}\text { Center } \\
\text { Number }\end{array}$} & Atomic & \multirow{2}{*}{$\begin{array}{l}\text { Atomic } \\
\text { Type }\end{array}$} & \multicolumn{2}{|c|}{ Coordinates (Angstroms } \\
\hline & Number & & $\mathrm{X}$ & Z \\
\hline 1 & 7 & 2.115209 & -0.411375 & 0.191658 \\
\hline 2 & 6 & 1.498165 & -1.011034 & -1.080457 \\
\hline 3 & 6 & 3.016771 & -1.504513 & 0.733350 \\
\hline 4 & 6 & 2.887733 & 0.910893 & -0.020431 \\
\hline 5 & 1 & 0.840265 & -1.755900 & -0.591581 \\
\hline 6 & 1 & 2.354964 & -2.056655 & 1.404473 \\
\hline 7 & 1 & 3.835493 & -1.084958 & 1.322985 \\
\hline 8 & 6 & 2.610521 & -1.883075 & -1.660859 \\
\hline 9 & 6 & 3.475465 & -2.357826 & -0.455700 \\
\hline 10 & 1 & 3.293743 & -3.416759 & -0.236533 \\
\hline 11 & 1 & 4.550951 & -2.248438 & -0.645003 \\
\hline 12 & 1 & 2.157809 & -2.738589 & -2.180217 \\
\hline 13 & 1 & 3.244407 & -1.377666 & $5-2.399997$ \\
\hline 14 & 6 & 1.911009 & 1.967323 & -0.526987 \\
\hline 15 & 6 & 3.460384 & 1.374061 & 1.322691 \\
\hline 16 & 6 & 4.017521 & 0.753427 & -1.036265 \\
\hline 17 & 1 & 1.187270 & 2.223094 & 0.250316 \\
\hline 18 & 1 & 2.482223 & 2.866129 & -0.793446 \\
\hline 19 & 1 & .382586 & 1.602296 & -1.412701 \\
\hline 20 & 1 & 2.709818 & 1.257720 & 2.110809 \\
\hline 21 & 1 & 4.374612 & 0.836136 & 1.600519 \\
\hline 22 & 1 & 3.721659 & 2.436408 & 1.237041 \\
\hline 23 & 1 & 3.626009 & 0.617677 & -2.049062 \\
\hline 24 & 1 & 4.608901 & 1.678278 & -1.020810 \\
\hline 25 & 1 & 4.694262 & -0.072965 & -0.790441 \\
\hline 26 & 3 & -0.300467 & -0.079812 & $2-0.203973$ \\
\hline 27 & 8 & 1.083781 & -0.151747 & 1.088674 \\
\hline 28 & 8 & -1.281055 & 1.598458 & $3-0.134195$ \\
\hline 29 & 6 & -1.622929 & 2.444161 & -1.244731 \\
\hline 30 & 6 & -2.095410 & 3.756951 & -0.628208 \\
\hline 31 & 6 & -1.261174 & 3.817286 & $5 \quad 0.654350$ \\
\hline 32 & 6 & -1.266307 & 2.356731 & 1.087835 \\
\hline 33 & 1 & -0.726139 & 2.588375 & -1.864518 \\
\hline 34 & 1 & -2.392652 & 1.936772 & -1.840126 \\
\hline 35 & 1 & -1.933978 & 4.609758 & -1.295500 \\
\hline 36 & 1 & -3.164322 & 3.700114 & -0.383302 \\
\hline 37 & 1 & -0.238441 & 4.147392 & 0.429485 \\
\hline 38 & 1 & -1.684809 & 4.478063 & 1.417668 \\
\hline 39 & 1 & -0.385317 & 2.042450 & 1.661565 \\
\hline 40 & 1 & -2.174150 & 2.117064 & 1.662294 \\
\hline
\end{tabular}




$\begin{array}{rrrrrr}41 & 7 & 0 & -1.833231 & -1.510579 & -0.231121 \\ 42 & 1 & 0 & -1.310436 & -2.217337 & -0.747111 \\ 43 & 6 & 0 & -3.010638 & -1.190475 & -1.070067 \\ 44 & 6 & 0 & -2.106573 & -2.058615 & 1.116872 \\ 45 & 1 & 0 & -2.599369 & -0.669378 & -1.949339 \\ 46 & 6 & 0 & -3.732354 & -2.445798 & -1.565041 \\ 47 & 6 & 0 & -3.981318 & -0.239700 & -0.378410 \\ 48 & 1 & 0 & -3.104722 & -2.527867 & 1.140217 \\ 49 & 6 & 0 & -1.064161 & -3.127105 & 1.430397 \\ 50 & 6 & 0 & -2.051884 & -0.952470 & 2.169615 \\ 51 & 1 & 0 & -3.040946 & -3.102220 & -2.110389 \\ 52 & 1 & 0 & -4.161904 & -3.012934 & -0.727501 \\ 53 & 1 & 0 & -4.551869 & -2.174491 & -2.243561 \\ 54 & 1 & 0 & -3.465903 & 0.660049 & -0.023739 \\ 55 & 1 & 0 & -4.762314 & 0.055173 & -1.090909 \\ 56 & 1 & 0 & -4.476545 & -0.727260 & 0.472586 \\ 57 & 1 & 0 & -1.121996 & -3.957879 & 0.713237 \\ 58 & 1 & 0 & -0.060309 & -2.680378 & 1.384427 \\ 59 & 1 & 0 & -1.220600 & -3.531427 & 2.438036 \\ 60 & 1 & 0 & -1.045925 & -0.508093 & 2.179795 \\ 61 & 1 & 0 & -2.784747 & -0.163752 & 1.970745 \\ 62 & 1 & 0 & -2.260740 & -1.369890 & 3.164191 \\ ----------------------------------------------------------\end{array}$

\section{Thermodynamics:}

Zero-point correction $=$

Thermal correction to Energy=

Thermal correction to Enthalpy=

Thermal correction to Gibbs Free Energy=

Sum of electronic and zero-point Energies=

Sum of electronic and thermal Energies=

Sum of electronic and thermal Enthalpies=

Sum of electronic and thermal Free Energies=

\subsection{8 (Hartree/Particle)} 0.592518

0.593462

0.506391

$-975.934862$

$-975.906712$

$-975.905768$

$-975.992839$ 
Structure 21 exo - M06-2x/jul-cc-pVDZ

Input:

$\# \mathrm{~m} 062 \mathrm{x} / \mathrm{jul}-\mathrm{cc}-\mathrm{pvDz}$ opt=(calcfc,noeigen,z-matrix $)$ optcyc $=50$ freq scrf $=(\mathrm{pcm}$,solvent=THF $)$

Optimized Structure:

\begin{tabular}{|c|c|c|c|c|}
\hline \multirow{2}{*}{$\begin{array}{l}\text { Center } \\
\text { Number }\end{array}$} & Atomic & \multirow{2}{*}{$\begin{array}{l}\text { Atomic } \\
\text { Type }\end{array}$} & \multicolumn{2}{|c|}{ Coordinates (Angstroms } \\
\hline & Number & & $\mathrm{X}$ & Z \\
\hline 1 & 7 & 2.105378 & -0.893292 & 0.017918 \\
\hline 2 & 8 & 1.133508 & -0.852379 & 1.016851 \\
\hline 3 & 6 & 3.187872 & 0.154952 & 0.316232 \\
\hline 4 & 6 & 2.671251 & -2.290230 & 0.020080 \\
\hline 5 & 6 & 1.398680 & -0.715907 & -1.321191 \\
\hline 6 & 6 & 1.637865 & -3.135311 & -0.720089 \\
\hline 7 & 6 & 0.776563 & -2.107826 & -1.504419 \\
\hline 8 & 1 & 2.833245 & -2.594063 & 1.057112 \\
\hline 9 & 1 & 3.626990 & -2.273175 & -0.513437 \\
\hline 10 & 1 & 1.029288 & -3.713969 & -0.014412 \\
\hline 11 & 1 & 2.139843 & -3.847984 & -1.385405 \\
\hline 12 & 1 & -0.248839 & -2.157727 & $7-1.116672$ \\
\hline 13 & 1 & 0.716647 & -2.350039 & -2.574617 \\
\hline 14 & 1 & 207138 & -0.624952 & -2.061842 \\
\hline 15 & 6 & 3.944175 & -0.272989 & 1.574778 \\
\hline 16 & 6 & 4.162815 & 0.315364 & -0.853131 \\
\hline 17 & 6 & 2.503457 & 1.493654 & 0.570141 \\
\hline 18 & 1 & 4.634149 & -1.102986 & $5 \quad 1.378686$ \\
\hline 19 & 1 & 535944 & 0.578182 & 1.934479 \\
\hline 20 & 1 & 3.234874 & -0.564159 & 2.357090 \\
\hline 21 & 1 & 4.579821 & -0.637465 & -1.198413 \\
\hline 22 & 1 & 3.691415 & 0.824247 & -1.700517 \\
\hline 23 & 1 & 5.000149 & 0.935512 & -0.507193 \\
\hline 24 & 1 & 1.846603 & 1.746153 & -0.266966 \\
\hline 25 & 1 & 1.914077 & 1.455191 & 1.490885 \\
\hline 26 & 1 & 3.278279 & 2.265422 & 0.670477 \\
\hline 27 & 3 & -0.214886 & -0.018294 & $4 \quad-0.028481$ \\
\hline 28 & 8 & -0.629662 & 1.940623 & -0.104761 \\
\hline 29 & 6 & -0.471811 & 2.586608 & $\begin{array}{l}3 \\
-1.382921\end{array}$ \\
\hline 30 & 6 & -0.442650 & 4.088545 & $5-1.098003$ \\
\hline 31 & 6 & 0.062907 & 4.139245 & 0.346864 \\
\hline 32 & 6 & -0.625163 & 2.919799 & 0.942751 \\
\hline 33 & 1 & 0.462803 & 2.220112 & -1.827893 \\
\hline 34 & 1 & -1.306720 & 2.287668 & $\begin{array}{l}3 \\
-2.031029\end{array}$ \\
\hline 35 & 1 & 0.199843 & 4.626572 & -1.802501 \\
\hline 36 & 1 & -1.453738 & 4.511486 & $5-1.157563$ \\
\hline 37 & 1 & 1.152670 & 4.015955 & 0.382717 \\
\hline 38 & 1 & -0.206179 & 5.065046 & $5 \quad 0.866218$ \\
\hline 39 & 1 & -0.108406 & 2.486848 & 1.807268 \\
\hline 40 & 1 & -1.664000 & 3.153293 & 1.224888 \\
\hline
\end{tabular}




$\begin{array}{rrrrrr}41 & 7 & 0 & -2.286246 & -0.788328 & -0.247623 \\ 42 & 1 & 0 & -2.247170 & -0.983815 & -1.247850 \\ 43 & 6 & 0 & -3.246922 & 0.324566 & -0.069768 \\ 44 & 6 & 0 & -2.679785 & -2.044082 & 0.449033 \\ 45 & 1 & 0 & -2.819910 & 1.166073 & -0.629763 \\ 46 & 6 & 0 & -4.635670 & 0.035439 & -0.643877 \\ 47 & 6 & 0 & -3.312494 & 0.736869 & 1.396558 \\ 48 & 1 & 0 & -3.662475 & -1.886610 & 0.916392 \\ 49 & 6 & 0 & -2.839642 & -3.198730 & -0.536117 \\ 50 & 6 & 0 & -1.685442 & -2.377200 & 1.555703 \\ 51 & 1 & 0 & -4.567895 & -0.221486 & -1.709756 \\ 52 & 1 & 0 & -5.133558 & -0.791585 & -0.119832 \\ 53 & 1 & 0 & -5.274362 & 0.923632 & -0.547805 \\ 54 & 1 & 0 & -2.304927 & 0.917270 & 1.791112 \\ 55 & 1 & 0 & -3.901145 & 1.657561 & 1.500296 \\ 56 & 1 & 0 & -3.792628 & -0.035331 & 2.013081 \\ 57 & 1 & 0 & -3.587683 & -2.956436 & -1.303495 \\ 58 & 1 & 0 & -1.888436 & -3.427285 & -1.036228 \\ 59 & 1 & 0 & -3.169247 & -4.103337 & -0.009284 \\ 60 & 1 & 0 & -0.677795 & -2.520902 & 1.144143 \\ 61 & 1 & 0 & -1.626298 & -1.553602 & 2.279590 \\ 62 & 1 & 0 & -1.998437 & -3.285639 & 2.088951 \\ ----------------------------------------------------------\end{array}$

\section{Thermodynamics:}

Zero-point correction $=$

Thermal correction to Energy=

Thermal correction to Enthalpy=

Thermal correction to Gibbs Free Energy=

Sum of electronic and zero-point Energies=

Sum of electronic and thermal Energies=

Sum of electronic and thermal Enthalpies=

Sum of electronic and thermal Free Energies=
0.563862 (Hartree/Particle) 0.592358

0.593302

0.505783

$-975.940862$

$-975.912366$

$-975.911422$

$-975.998941$ 


\section{Structure 21 exo-m - M06-2x/jul-cc-pVDZ}

Input:

$\#$ 062x/jul-cc-pvDz opt=(calcfc,noeigen,z-matrix $)$ optcyc $=50$ freq $\mathrm{scrf}=(\mathrm{pcm}$,solvent=THF $)$

Optimized Structure:

\begin{tabular}{|c|c|c|c|c|}
\hline \multirow{3}{*}{$\begin{array}{c}\text { Center } \\
\text { Number } \\
1\end{array}$} & \multirow{2}{*}{$\begin{array}{l}\text { Atomic } \\
\text { Number }\end{array}$} & \multirow{2}{*}{$\begin{array}{l}\text { Atomic } \\
\text { Type }\end{array}$} & \multicolumn{2}{|c|}{ Coordinates (Angstroms } \\
\hline & & & $\mathrm{X}$ & $\mathrm{Z}$ \\
\hline & 7 & 1.889363 & -0.973544 & -0.026378 \\
\hline 2 & 8 & 1.028083 & -0.675671 & 1.036653 \\
\hline 3 & 6 & 3.139415 & -0.086837 & 0.102379 \\
\hline 4 & 6 & 2.215511 & -2.432903 & 0.096438 \\
\hline 5 & 6 & 1.129202 & -0.798081 & -1.354856 \\
\hline 6 & 6 & 1.004510 & -3.111899 & -0.512411 \\
\hline 7 & 6 & 0.709672 & -2.235427 & -1.740598 \\
\hline 8 & 1 & 2.389611 & -2.660145 & 1.150872 \\
\hline 9 & 1 & 3.112493 & -2.652379 & -0.494249 \\
\hline 10 & 1 & 0.179404 & -3.086210 & 0.208993 \\
\hline 11 & 1 & 1.210846 & -4.158411 & -0.765622 \\
\hline 12 & 1 & -0.354317 & -2.312344 & -2.020978 \\
\hline 13 & 1 & 1.279435 & -2.623365 & -2.602978 \\
\hline 14 & 3 & -0.272627 & 0.103506 & -0.061015 \\
\hline 15 & 1 & 1.868569 & -0.441040 & -2.078134 \\
\hline 16 & 6 & 3.855792 & -0.450690 & 1.403717 \\
\hline 17 & 6 & 4.107429 & -0.256075 & -1.074218 \\
\hline 18 & 6 & 2.676640 & 1.367125 & 0.159609 \\
\hline 19 & 1 & 4.388539 & -1.406541 & 1.319765 \\
\hline 20 & 1 & 4.597180 & 0.327535 & 1.625087 \\
\hline 21 & 1 & 3.137522 & -0.502427 & 2.227805 \\
\hline 22 & 1 & 4.313951 & -1.304735 & -1.314123 \\
\hline 23 & 1 & 3.750466 & 0.247150 & -1.978246 \\
\hline 24 & 1 & 5.060506 & 0.208227 & -0.788810 \\
\hline 25 & 1 & 2.122158 & 1.556175 & 1.082709 \\
\hline 26 & 1 & 3.561 & 2.016905 & 0.130235 \\
\hline 27 & 1 & 2.033807 & 1.601097 & -0.696552 \\
\hline 28 & 8 & -0.684824 & 2.015568 & -0.057167 \\
\hline 29 & 6 & -0.557482 & 2.765789 & -1.278982 \\
\hline 30 & 6 & -0.388530 & 4.222921 & -0.855818 \\
\hline 31 & 6 & 0.288669 & 4.082126 & 0.510370 \\
\hline 32 & 6 & -0.420594 & 2.858484 & 1.075058 \\
\hline 33 & 1 & 0.318284 & 2.387962 & -1.824947 \\
\hline 34 & 1 & -1.453669 & 2.589194 & -1.887214 \\
\hline 35 & 1 & 0.202492 & 4.795746 & -1.577872 \\
\hline 36 & 1 & -1.368162 & 4.705851 & -0.745142 \\
\hline 37 & 1 & 1.360310 & 3.877563 & 0.389210 \\
\hline 38 & 1 & 0.166932 & 4.964403 & 1.147384 \\
\hline 39 & 1 & 0.177289 & 2.282866 & 1.791810 \\
\hline 40 & 1 & -1.376922 & 3.133616 & 1.54593 \\
\hline
\end{tabular}




$\begin{array}{rrrrrr}41 & 7 & 0 & -2.010550 & -1.002308 & 0.000093 \\ 42 & 1 & 0 & -1.544460 & -1.874060 & -0.246821 \\ 43 & 6 & 0 & -2.950770 & -0.692607 & -1.093522 \\ 44 & 6 & 0 & -2.582671 & -1.148942 & 1.355175 \\ 45 & 1 & 0 & -2.310601 & -0.520563 & -1.974351 \\ 46 & 6 & 0 & -3.894548 & -1.853242 & -1.414180 \\ 47 & 6 & 0 & -3.720250 & 0.594864 & -0.817946 \\ 48 & 1 & 0 & -3.672763 & -1.300480 & 1.289969 \\ 49 & 6 & 0 & -1.964443 & -2.367742 & 2.030775 \\ 50 & 6 & 0 & -2.307569 & 0.108175 & 2.178484 \\ 51 & 1 & 0 & -3.323352 & -2.759853 & -1.656511 \\ 52 & 1 & 0 & -4.554668 & -2.076296 & -0.564178 \\ 53 & 1 & 0 & -4.527862 & -1.607378 & -2.277026 \\ 54 & 1 & 0 & -3.031643 & 1.413318 & -0.576098 \\ 55 & 1 & 0 & -4.306556 & 0.869093 & -1.704010 \\ 56 & 1 & 0 & -4.421347 & 0.466962 & 0.018661 \\ 57 & 1 & 0 & -2.212735 & -3.288121 & 1.483312 \\ 58 & 1 & 0 & -0.871356 & -2.248876 & 2.055156 \\ 59 & 1 & 0 & -2.332840 & -2.468465 & 3.059355 \\ 60 & 1 & 0 & -1.220921 & 0.225023 & 2.308315 \\ 61 & 1 & 0 & -2.695893 & 1.004928 & 1.682257 \\ 62 & 1 & 0 & -2.771124 & 0.029132 & 3.171352 \\ -------------------------------------------------------------\end{array}$

\section{Thermodynamics:}

Zero-point correction $=$

Thermal correction to Energy=

Thermal correction to Enthalpy=

0.563569 (Hartree/Particle) 0.591858

0.592802

Thermal correction to Gibbs Free Energy $=0.505742$

Sum of electronic and zero-point Energies $=$

$-975.946849$

Sum of electronic and thermal Energies $=\quad-975.918560$

Sum of electronic and thermal Enthalpies $=\quad-975.917615$

Sum of electronic and thermal Free Energies $=\quad-976.004675$ 
Structure 21 $1_{\text {TS }}$ - M06-2x/jul-cc-pVDZ

Input:

$\#$ m062x/jul-cc-pvDz opt=(calcfc,noeigen,z-matrix,ts) optcyc=50 freq scr $\mathrm{f}=(\mathrm{pcm}$, solvent $=\mathrm{THF})$

Optimized Structure:

\begin{tabular}{|c|c|c|c|c|}
\hline \multirow{3}{*}{$\begin{array}{l}\text { Center } \\
\text { Number } \\
-1\end{array}$} & \multirow{2}{*}{$\begin{array}{l}\text { Atomic } \\
\text { Number }\end{array}$} & \multirow{2}{*}{$\begin{array}{r}\text { Atomic } \\
\text { Type }\end{array}$} & \multicolumn{2}{|c|}{ Coordinates (Angstroms } \\
\hline & & & $\mathrm{X}$ & Z \\
\hline & 7 & 2.105378 & -0.893292 & 0.017918 \\
\hline 2 & 8 & 1.133508 & -0.852379 & 1.016851 \\
\hline 3 & 6 & 3.187872 & 0.154952 & 0.316232 \\
\hline 4 & 6 & 2.671251 & -2.290230 & 0.020080 \\
\hline 5 & 6 & 1.398680 & -0.715907 & -1.321191 \\
\hline 6 & 6 & 1.637865 & -3.135311 & -0.720089 \\
\hline 7 & 6 & 0.776563 & -2.107826 & -1.504419 \\
\hline 8 & 1 & 2.833245 & -2.594063 & 1.057112 \\
\hline 9 & 1 & 3.626990 & -2.273175 & -0.513437 \\
\hline 10 & 1 & 1.029288 & -3.713969 & -0.014412 \\
\hline 11 & 1 & 2.139843 & -3.847984 & -1.385405 \\
\hline 12 & 1 & -0.248839 & -2.157727 & -1.116672 \\
\hline 13 & 1 & 0.716647 & -2.350039 & -2.574617 \\
\hline 14 & 1 & 2.207138 & -0.624952 & -2.061842 \\
\hline 15 & 6 & 3.944175 & -0.272989 & 1.574778 \\
\hline 16 & 6 & 4.162815 & 0.315364 & -0.853131 \\
\hline 17 & 6 & 2.503457 & 1.493654 & 0.570141 \\
\hline 18 & 1 & 4.634149 & -1.102986 & 1.378686 \\
\hline 19 & 1 & 4.535944 & 0.578182 & 1.934479 \\
\hline 20 & 1 & 3.234874 & -0.564159 & 2.357090 \\
\hline 21 & 1 & 4.579821 & -0.637465 & -1.198413 \\
\hline 22 & 1 & 3.691415 & 0.824247 & -1.700517 \\
\hline 23 & 1 & 5.000149 & 0.935512 & -0.507193 \\
\hline 24 & 1 & 1.846603 & 1.746153 & -0.266966 \\
\hline 25 & 1 & 1.914077 & 1.455191 & 1.490885 \\
\hline 26 & 1 & 3.278279 & 2.265422 & 0.670477 \\
\hline 27 & 3 & -0.214886 & -0.018294 & -0.028481 \\
\hline 28 & 8 & -0.629662 & 1.940623 & -0.104761 \\
\hline 29 & 6 & -0.471811 & 2.586608 & -1.382921 \\
\hline 30 & 6 & -0.442650 & 4.088545 & -1.098003 \\
\hline 31 & 6 & 0.062907 & 4.139245 & 0.346864 \\
\hline 32 & 6 & -0.625163 & 2.919799 & 0.942751 \\
\hline 33 & 1 & 0.462803 & 2.220112 & -1.827893 \\
\hline 34 & 1 & -1.306720 & 2.287668 & -2.031029 \\
\hline 35 & 1 & 0.199843 & 4.626572 & -1.802501 \\
\hline 36 & 1 & -1.453738 & 4.511486 & -1.157563 \\
\hline 37 & 1 & 1.152670 & 4.015955 & 0.382717 \\
\hline 38 & 1 & -0.206179 & 5.065046 & 0.866218 \\
\hline 39 & 1 & -0.108406 & 2.486848 & 1.807268 \\
\hline
\end{tabular}




$\begin{array}{rrrrrr}40 & 1 & 0 & -1.664000 & 3.153293 & 1.224888 \\ 41 & 7 & 0 & -2.286246 & -0.788328 & -0.247623 \\ 42 & 1 & 0 & -2.247170 & -0.983815 & -1.247850 \\ 43 & 6 & 0 & -3.246922 & 0.324566 & -0.069768 \\ 44 & 6 & 0 & -2.679785 & -2.044082 & 0.449033 \\ 45 & 1 & 0 & -2.819910 & 1.166073 & -0.629763 \\ 46 & 6 & 0 & -4.635670 & 0.035439 & -0.643877 \\ 47 & 6 & 0 & -3.312494 & 0.736869 & 1.396558 \\ 48 & 1 & 0 & -3.662475 & -1.886610 & 0.916392 \\ 49 & 6 & 0 & -2.839642 & -3.198730 & -0.536117 \\ 50 & 6 & 0 & -1.685442 & -2.377200 & 1.555703 \\ 51 & 1 & 0 & -4.567895 & -0.221486 & -1.709756 \\ 52 & 1 & 0 & -5.133558 & -0.791585 & -0.119832 \\ 53 & 1 & 0 & -5.274362 & 0.923632 & -0.547805 \\ 54 & 1 & 0 & -2.304927 & 0.917270 & 1.791112 \\ 55 & 1 & 0 & -3.901145 & 1.657561 & 1.500296 \\ 56 & 1 & 0 & -3.792628 & -0.035331 & 2.013081 \\ 57 & 1 & 0 & -3.587683 & -2.956436 & -1.303495 \\ 58 & 1 & 0 & -1.888436 & -3.427285 & -1.036228 \\ 59 & 1 & 0 & -3.169247 & -4.103337 & -0.009284 \\ 60 & 1 & 0 & -0.677795 & -2.520902 & 1.144143 \\ 61 & 1 & 0 & -1.626298 & -1.553602 & 2.279590 \\ 62 & 1 & 0 & -1.998437 & -3.285639 & 2.088951 \\ -----------------------------------------------------\end{array}$

Thermodynamics:

Zero-point correction $=$

Thermal correction to Energy=

Thermal correction to Enthalpy=

Thermal correction to Gibbs Free Energy=

Sum of electronic and zero-point Energies=

Sum of electronic and thermal Energies=

Sum of electronic and thermal Enthalpies=

Sum of electronic and thermal Free Energies=
0.563862 (Hartree/Particle) 0.592358
0.593302

0.505783

$-975.940862$

$-975.912366$

$-975.911422$

$-975.998941$ 
Structure 22 - M06-2x/jul-cc-pVDZ

Input:

$\# \mathrm{~m} 062 \mathrm{x} / \mathrm{jul}-\mathrm{cc}-\mathrm{pvDz}$ opt=(calcfc,noeigen,z-matrix $)$ optcyc $=50$ freq scrf $=(\mathrm{pcm}$,solvent=THF $)$

Optimized Structure:

\begin{tabular}{|c|c|c|c|c|}
\hline Center & Atomic & Atomic & $\mathrm{Co}$ & \\
\hline Number & Number & Type & $\mathrm{X}$ & Z \\
\hline 1 & 7 & 0.174581 & -0.333144 & 1.956606 \\
\hline 2 & 8 & 0.156216 & -0.196417 & 0.554621 \\
\hline 3 & 6 & 0.191703 & -1.840237 & 2.271263 \\
\hline 4 & 6 & 1.409870 & 0.367309 & 2.466723 \\
\hline 5 & 6 & -1.040155 & 0.424289 & 2.538211 \\
\hline 6 & 6 & 0.944042 & 1.780003 & 2.763381 \\
\hline 7 & 6 & -0.423817 & 1.534654 & 3.399281 \\
\hline 8 & 1 & 2.201513 & 0.281809 & 1.713772 \\
\hline 9 & 1 & 1.715929 & -0.113030 & 3.400953 \\
\hline 10 & 1 & 0.856109 & 2.350170 & 1.831633 \\
\hline 11 & 1 & 1.649852 & 2.297897 & 3.423952 \\
\hline 12 & 1 & -1.037486 & 2.447217 & 3.399427 \\
\hline 13 & 1 & -0.270450 & 1.250483 & 4.459294 \\
\hline 14 & 1 & -1.575505 & -0.292444 & 3.171731 \\
\hline 15 & 6 & 1.341342 & -2.496346 & 1.504054 \\
\hline 16 & 6 & 0.373297 & -2.086769 & 3.772914 \\
\hline 17 & 6 & -1.128973 & -2.456178 & 1.806771 \\
\hline 18 & 1 & 2.291917 & -1.971638 & 1.660207 \\
\hline 19 & 1 & 1.459874 & -3.524371 & 1.870389 \\
\hline 20 & 1 & 1.123583 & -2.542651 & 0.430927 \\
\hline 21 & 1 & 1.421125 & -2.005588 & 4.082393 \\
\hline 22 & 1 & -0.232345 & -1.400057 & 4.374862 \\
\hline 23 & 1 & 0.046112 & -3.110581 & 3.992407 \\
\hline 24 & 1 & -1.957300 & -2.204699 & 2.477609 \\
\hline 25 & 1 & -1.375645 & -2.119366 & 0.796630 \\
\hline 26 & 1 & -1.015129 & -3.548193 & 1.803408 \\
\hline 27 & 3 & -1.567747 & 0.597796 & 0.500489 \\
\hline 28 & 8 & -3.206767 & -0.590905 & 0.052271 \\
\hline 29 & 6 & -4.176333 & -0.381002 & 1.097786 \\
\hline 30 & 6 & -5.426750 & -1.120661 & 0.642619 \\
\hline 31 & 6 & -4.817700 & -2.318294 & -0.088139 \\
\hline 32 & 6 & -3.621780 & -1.680777 & -0.788695 \\
\hline 33 & 1 & -3.776294 & -0.788567 & 2.036478 \\
\hline 34 & 1 & -4.314583 & 0.700632 & 1.218721 \\
\hline 35 & 1 & -6.070022 & -1.403307 & 1.482169 \\
\hline 36 & 1 & -6.006990 & -0.501060 & -0.053668 \\
\hline 37 & 1 & -4.474290 & -3.069097 & 0.635737 \\
\hline 38 & 1 & -5.503557 & -2.798824 & -0.793396 \\
\hline 39 & 1 & -2.783431 & -2.377525 & -0.910869 \\
\hline 40 & 1 & -3.896445 & -1.275925 & -1.774586 \\
\hline
\end{tabular}




\begin{tabular}{|c|c|c|c|c|c|}
\hline 41 & 7 & 0 & -2.234871 & 1.967720 & -0.919889 \\
\hline 42 & 1 & 0 & -3.232994 & 1.780281 & -0.836823 \\
\hline 43 & 6 & 0 & -1.805184 & 1.348674 & -2.192505 \\
\hline 44 & 6 & 0 & -2.020379 & 3.426098 & -0.794014 \\
\hline 45 & 1 & ( & -2.003933 & 0.275364 & -2.058232 \\
\hline 46 & 6 & 0 & -2.619289 & 1.815873 & -3.399695 \\
\hline 47 & 6 & 0 & -0.308153 & 1.525710 & -2.411459 \\
\hline 48 & 1 & 0 & -1.842881 & 3.864536 & -1.789028 \\
\hline 49 & 6 & 0 & -3.274488 & 4.065064 & -0.208974 \\
\hline 50 & 6 & 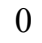 & -0.810423 & 3.710039 & 0.090320 \\
\hline 51 & 1 & 0 & -3.691453 & 1.643236 & -3.233583 \\
\hline 52 & 1 & 0 & -2.465566 & 2.884240 & -3.602769 \\
\hline 53 & 1 & 0 & -2.318533 & 1.258345 & -4.296704 \\
\hline 54 & 1 & 0 & 0.254138 & 1.205837 & -1.525128 \\
\hline 55 & 1 & 0 & 0.019743 & 0.922638 & -3.267434 \\
\hline 56 & 1 & 0 & -0.057117 & 2.575148 & -2.620879 \\
\hline 57 & 1 & 0 & -4.134681 & 3.931252 & -0.879105 \\
\hline 58 & 1 & 0 & -3.509583 & 3.603776 & 0.762151 \\
\hline 59 & 1 & 0 & -3.124457 & 5.139635 & -0.048783 \\
\hline 60 & 1 & 0 & -1.004791 & 3.332357 & 1.104578 \\
\hline 61 & 1 & 0 & 0.092103 & 3.216180 & -0.290639 \\
\hline 62 & 1 & 0 & -0.618396 & 4.790139 & 0.145720 \\
\hline 63 & 7 & 0 & 3.355066 & 0.415111 & -0.389697 \\
\hline 64 & 6 & 0 & 4.767277 & 0.137445 & -0.481505 \\
\hline 65 & 6 & 0 & 3.010346 & 1.824319 & -0.325959 \\
\hline 66 & 1 & 0 & 5.328034 & 0.991514 & -0.920169 \\
\hline 67 & 6 & 0 & 5.428099 & -0.164613 & 0.881593 \\
\hline 68 & 6 & c & 5.011343 & -1.062550 & -1.402308 \\
\hline 69 & 1 & 0 & 1.928286 & 1.862620 & -0.089844 \\
\hline 70 & 6 & 0 & 3.705089 & 2.649288 & 0.775575 \\
\hline 71 & 6 & 0 & 3.175535 & 2.561520 & -1.671249 \\
\hline 72 & 1 & 0 & 6.511750 & -0.343908 & 0.784123 \\
\hline 73 & 1 & 0 & 5.276635 & 0.660722 & 1.586739 \\
\hline 74 & 1 & 0 & 4.966382 & -1.066765 & 1.314194 \\
\hline 75 & 1 & 0 & 4.438785 & -1.925908 & -1.023912 \\
\hline 76 & 1 & 0 & 4.663609 & -0.840463 & -2.420498 \\
\hline 77 & 1 & 0 & 6.073427 & -1.349039 & -1.443064 \\
\hline 78 & 1 & 0 & 4.786342 & 2.721921 & 0.584881 \\
\hline 79 & 1 & 0 & 3.301699 & 3.673258 & 0.810576 \\
\hline 80 & 1 & 0 & 3.561761 & 2.187639 & 1.761192 \\
\hline 81 & 1 & 0 & 4.233198 & 2.582127 & -1.974470 \\
\hline 82 & 1 & 0 & 2.607006 & 2.039232 & -2.451914 \\
\hline 83 & 1 & 0 & 2.822793 & 3.604303 & -1.614378 \\
\hline 84 & 3 & 0 & 1.710574 & -0.479913 & -0.506752 \\
\hline 85 & 8 & 0 & 1.034882 & -1.522996 & -2.015878 \\
\hline 86 & 6 & 0 & -0.328589 & -1.949596 & -1.997636 \\
\hline 87 & 6 & 0 & -0.283024 & -3.485528 & -1.889185 \\
\hline 88 & 6 & 0 & 1.174822 & -3.840276 & -2.267676 \\
\hline 89 & 6 & 0 & 1.740599 & -2.510819 & -2.768863 \\
\hline 90 & 1 & 0 & -0.800730 & -1.447441 & -1.147610 \\
\hline 91 & 1 & 0 & -0.811464 & -1.633796 & -2.937328 \\
\hline 92 & 1 & 0 & -0.518807 & -3.819134 & -0.871493 \\
\hline 93 & 1 & 0 & -1.006717 & -3.946639 & -2.56990 \\
\hline
\end{tabular}




$\begin{array}{llllll}94 & 1 & 0 & 1.733655 & -4.176434 & -1.386211 \\ 95 & 1 & 0 & 1.234985 & -4.624538 & -3.029246 \\ 96 & 1 & 0 & 2.809265 & -2.367851 & -2.589919 \\ 97 & 1 & 0 & 1.525438 & -2.364412 & -3.839498\end{array}$

\section{Thermodynamics:}

Zero-point correction $=$

Thermal correction to Energy=

Thermal correction to Enthalpy=

Thermal correction to Gibbs Free Energy=

Sum of electronic and zero-point Energies=

Sum of electronic and thermal Energies=

Sum of electronic and thermal Enthalpies=

Sum of electronic and thermal Free Energies=
0.878922 (Hartree/Particle)

0.924949

0.925893

0.800550

$-1507.304900$

$-1507.258873$

$-1507.257929$

$-1507.383272$ 


\section{Structure 22 $2_{\text {TS-A }}-$ M06-2x/jul-cc-pVDZ}

Input: $\# \mathrm{~m} 062 \mathrm{x} / \mathrm{jul}-\mathrm{cc}-\mathrm{pvDz}$ opt $=($ calcfc, noeigen, $\mathrm{z}$-matrix,ts $)$ optcyc $=50$ freq scr
$\mathrm{f}=(\mathrm{pcm}$,solvent $=\mathrm{THF})$

Optimized Structure:

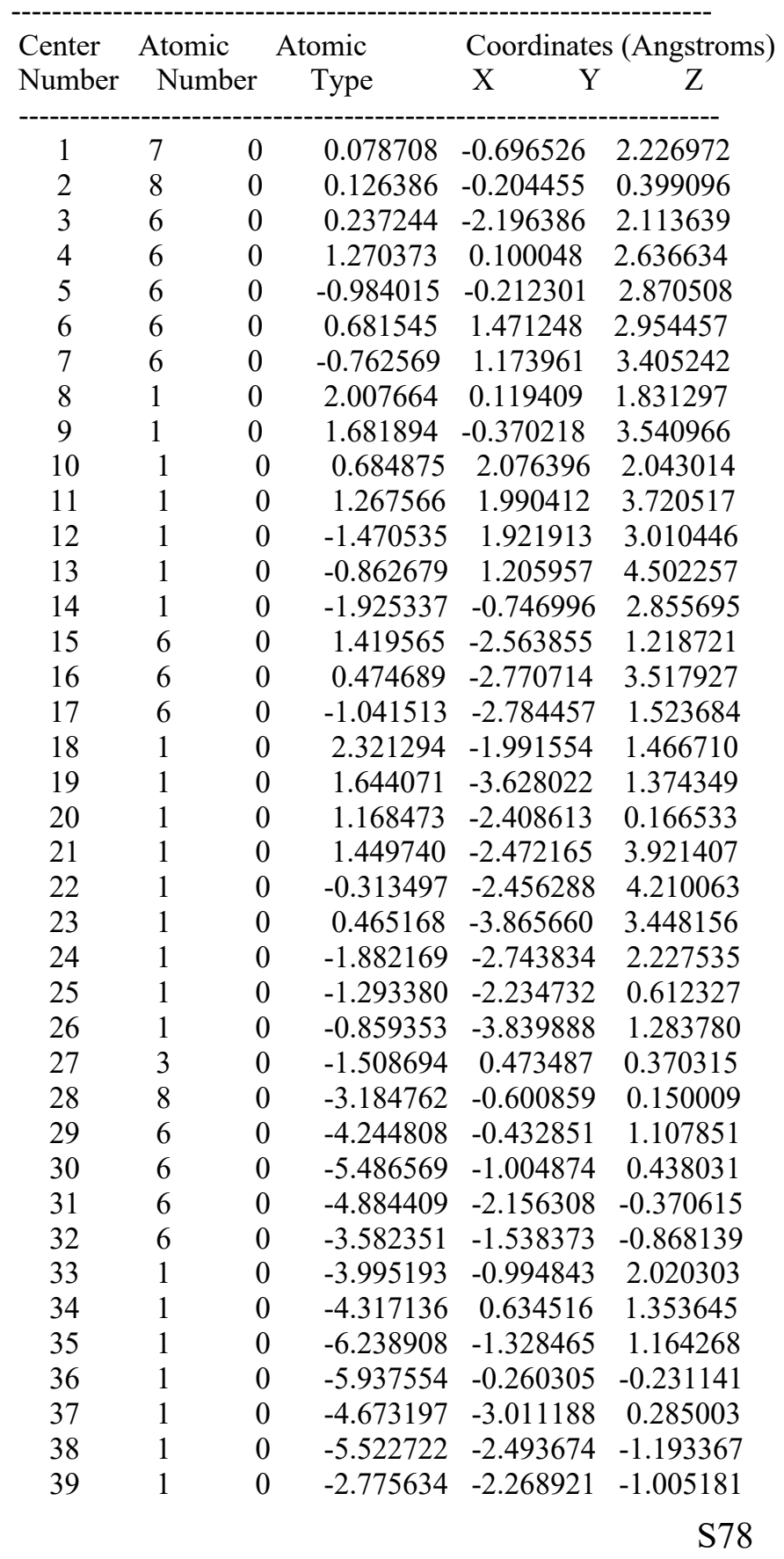




\begin{tabular}{|c|c|c|c|c|c|}
\hline 40 & 1 & 0 & -3.733207 & -0.988688 & -1.809288 \\
\hline 41 & 7 & 0 & -2.227308 & 2.016084 & -0.824387 \\
\hline 42 & 1 & 0 & -3.227013 & 1.825239 & -0.766529 \\
\hline 43 & 6 & 0 & -1.773874 & 1.434859 & -2.111785 \\
\hline 44 & 6 & 0 & -2.029245 & 3.472794 & -0.66195 \\
\hline 45 & 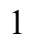 & U & -1.884927 & 0.346318 & -1.98763 \\
\hline 46 & 6 & 0 & -2.649851 & 1.857363 & -3.29103 \\
\hline 47 & ( & 0 & -0.297524 & 1.708743 & -2.3645 \\
\hline 48 & 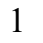 & 0 & -1.897531 & 3.943491 & -1.64972 \\
\hline 49 & 6 & ) & -3.272538 & 4.071493 & -0.014460 \\
\hline 50 & 6 & 0 & -0.792285 & 3.759611 & 0.184247 \\
\hline 51 & 1 & 0 & -3.702667 & 1.602473 & -3.10583 \\
\hline 52 & 1 & 0 & -2.581115 & 2.938546 & -3.47328 \\
\hline 53 & . & 0 & -2.328926 & 1.341493 & -4.20571 \\
\hline 54 & 1. & 0 & 0.298518 & 1.389725 & -1.50013 \\
\hline 55 & 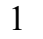 & 0 & 0.033683 & 1.139975 & -3.24287 \\
\hline 56 & . & 0 & -0.113877 & 2.774264 & -2.56186 \\
\hline 57 & 1 & ) & -4.152979 & 3.947735 & -0.65979 \\
\hline 58 & 1 & 0 & -3.470103 & 3.574641 & 0.947614 \\
\hline 59 & 1 & 0 & -3.132408 & 5.142074 & 0.17776 \\
\hline 60 & 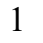 & 0 & -0.953790 & 3.391820 & 1.20804 \\
\hline 61 & 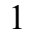 & 0 & 0.101075 & 3.268802 & -0.21847 \\
\hline 62 & 1 & 0 & -0.605890 & 4.841138 & 0.23187 \\
\hline 63 & 7 & ) & 3.315060 & 0.621744 & -0.24781 \\
\hline 64 & 6 & 0 & 4.740173 & 0.465429 & -0.39532 \\
\hline 65 & ( & ) & 2.870691 & 1.983382 & -0.018536 \\
\hline 66 & 1 & 0 & 5.228229 & 1.404483 & -0.738529 \\
\hline 67 & 6 & ) & 5.459726 & 0.057390 & 0.91015 \\
\hline 68 & & 0 & 5.047241 & -0.598102 & -1.45526 \\
\hline 69 & 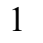 & 0 & 1.787828 & 1.906184 & 0.19105 \\
\hline 70 & 6 & 0 & 3.486823 & 2.709523 & 1.19481 \\
\hline 71 & 6 & 0 & 3.005326 & 2.897463 & -1.25621 \\
\hline 72 & 1 & ) & 6.549614 & -0.039802 & 0.770814 \\
\hline 73 & 1 & 0 & 5.276221 & 0.788493 & 1.706319 \\
\hline 74 & 1 & 0 & 5.064636 & -0.914050 & 1.24780 \\
\hline 75 & 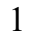 & 0 & 4.554470 & -1.540883 & -1.16454 \\
\hline 76 & 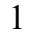 & 0 & 4.647334 & -0.290992 & -2.43164 \\
\hline 77 & 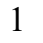 & 0 & 6.126359 & -0.792705 & -1.55557 \\
\hline 78 & 1 & 0 & 4.562393 & 2.886620 & 1.04119 \\
\hline 79 & 1 & ) & 3.006171 & 3.688246 & 1.35380 \\
\hline 80 & 1 & ) & 3.365311 & 2.112342 & 2.108874 \\
\hline 81 & 1 & 0 & 4.062985 & 3.043115 & -1.52308 \\
\hline 82 & 1 & 0 & 2.498014 & 2.435910 & -2.11388 \\
\hline 83 & 1 & 0 & 2.566676 & 3.893880 & -1.08060 \\
\hline 84 & 3 & 0 & 1.694761 & -0.346883 & -0.52736 \\
\hline 85 & 8 & 0 & 1.237061 & -1.409741 & -2.19586 \\
\hline 86 & 6 & 0 & -0.116688 & -1.875154 & -2.21632 \\
\hline 87 & 6 & 0 & -0.032611 & -3.415036 & -2.20898 \\
\hline 88 & 6 & 0 & 1.452221 & -3.709382 & -2.522809 \\
\hline 89 & 6 & 0 & 1.986487 & -2.347215 & -2.96469 \\
\hline 90 & 1 & 0 & -0.589786 & -1.435174 & -1.3299 \\
\hline 91 & 1 & 0 & -0.602239 & -1.511483 & -3.1379 \\
\hline 92 & 1 & 0 & -0.319070 & -3.824681 & -1.2330 \\
\hline
\end{tabular}




$\begin{array}{rrrrrr}93 & 1 & 0 & -0.700103 & -3.846562 & -2.963197 \\ 94 & 1 & 0 & 1.980773 & -4.039737 & -1.620318 \\ 95 & 1 & 0 & 1.580502 & -4.475130 & -3.295329 \\ 96 & 1 & 0 & 3.047682 & -2.183859 & -2.757756 \\ 97 & 1 & 0 & 1.793265 & -2.174766 & -4.036697\end{array}$

\section{Thermodynamics:}

Zero-point correction $=$

Thermal correction to Energy=

Thermal correction to Enthalpy=

Thermal correction to Gibbs Free Energy=

Sum of electronic and zero-point Energies=

Sum of electronic and thermal Energies=

Sum of electronic and thermal Enthalpies=

Sum of electronic and thermal Free Energies=
0.876377 (Hartree/Particle)

0.922972

0.923916

0.795797

$-1507.275773$

$-1507.229178$

$-1507.228234$

$-1507.356353$ 


\section{Structure 22 2 TS-B - M06-2x/jul-cc-pVDZ}

Input: $\# \mathrm{~m} 062 \mathrm{x} / \mathrm{jul}-\mathrm{cc}-\mathrm{pvDz}$ opt $=($ calcfc, noeigen, $\mathrm{z}$-matrix,ts $)$ optcyc $=50$ freq scr
$\mathrm{f}=(\mathrm{pcm}$,solvent $=\mathrm{THF})$

Optimized Structure:

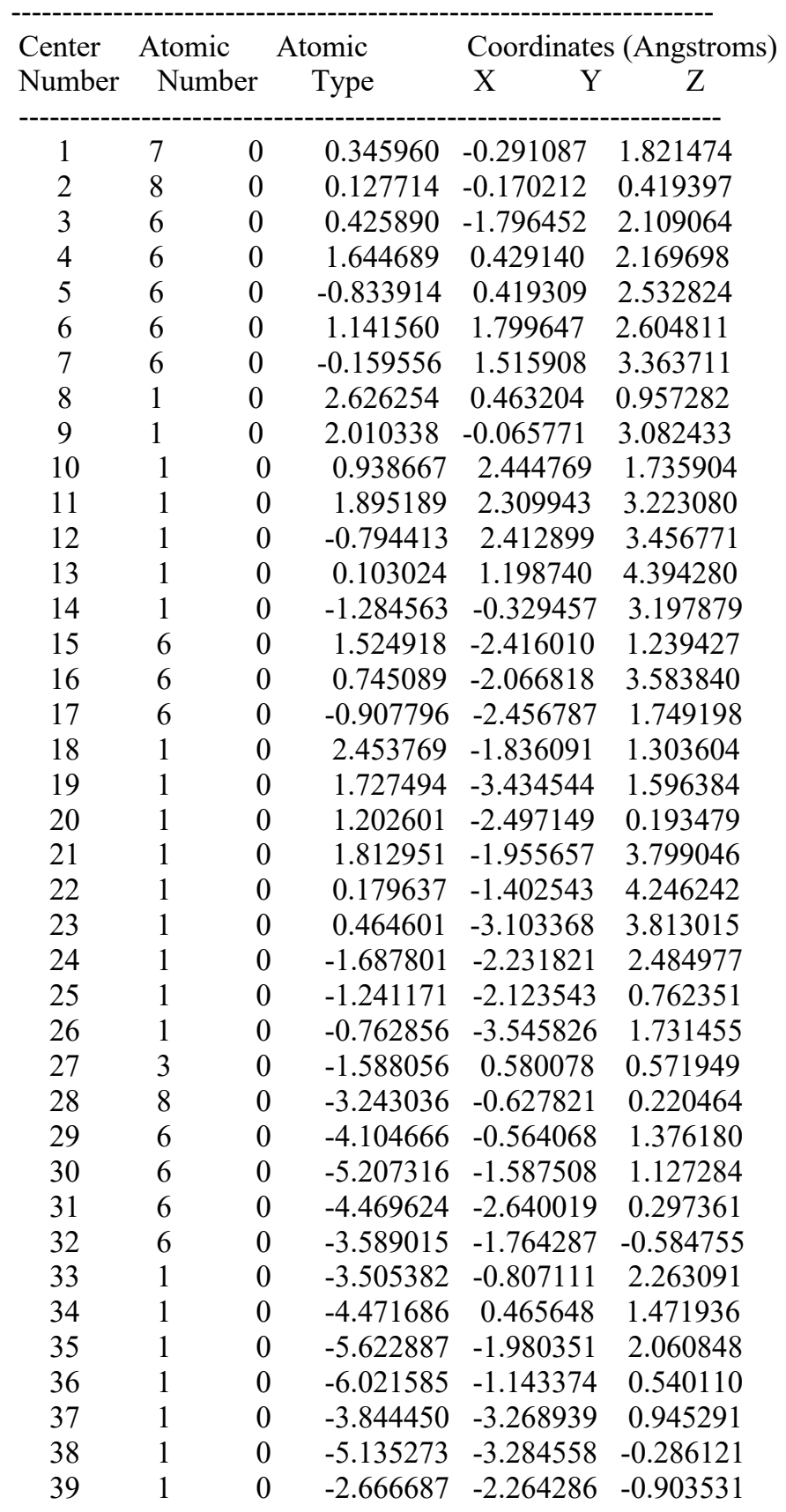




\begin{tabular}{|c|c|c|c|c|c|}
\hline 40 & 1 & 0 & -4.132833 & -1.416639 & -1.477005 \\
\hline 41 & 7 & 0 & -2.397165 & 1.940708 & -0.800343 \\
\hline 42 & 1 & 0 & -3.373966 & 1.704652 & -0.633675 \\
\hline 43 & 6 & 0 & -2.052108 & 1.369815 & -2.118586 \\
\hline 44 & 6 & 0 & -2.231571 & 3.402236 & -0.64894 \\
\hline 45 & 1 & 0 & -2.222703 & 0.288256 & -2.00426 \\
\hline 46 & 6 & 0 & -2.957682 & 1.856911 & -3.25128 \\
\hline 47 & ( & 0 & -0.576863 & 1.580355 & -2.43803 \\
\hline 48 & . & 0 & -2.145497 & 3.873991 & -1.6410 \\
\hline 49 & 6 & 0 & -3.463575 & 3.972284 & 0.044823 \\
\hline 50 & 6 & 0 & -0.972120 & 3.712259 & 0.15338 \\
\hline 51 & 1 & 0 & -4.013314 & 1.674072 & -3.00836 \\
\hline 52 & 1 & 0 & -2.825413 & 2.930126 & -3.44336 \\
\hline 53 & . & 0 & -2.722776 & 1.319899 & -4.17997 \\
\hline 54 & . & 0 & 0.047503 & 1.211620 & -1.61441 \\
\hline 55 & 1 & 0 & -0.309367 & 1.037947 & -3.35402 \\
\hline 56 & 1 & 0 & -0.352747 & 2.644273 & -2.60027 \\
\hline 57 & 1 & 0 & -4.364302 & 3.824227 & -0.56651 \\
\hline 58 & 1 & 0 & -3.609386 & 3.470680 & 1.013503 \\
\hline 59 & 1 & 0 & -3.343530 & 5.046497 & 0.23091 \\
\hline 60 & 1 & 0 & -1.068187 & 3.291819 & 1.16458 \\
\hline 61 & 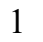 & 0 & -0.082374 & 3.269411 & -0.31072 \\
\hline 62 & 1 & ) & -0.820699 & 4.797674 & 0.23066 \\
\hline 63 & ? & 0 & 3.179227 & 0.552247 & -0.12380 \\
\hline 64 & 6 & 0 & 4.592887 & 0.184734 & -0.11395 \\
\hline 65 & 6 & 0 & 2.876456 & 1.910696 & -0.578284 \\
\hline 66 & 1 & 0 & 5.194661 & 0.986280 & -0.57594 \\
\hline 67 & 6 & 0 & 5.114399 & -0.009956 & 1.31481 \\
\hline 68 & ( & 0 & 4.825666 & -1.079772 & -0.94412 \\
\hline 69 & 1 & 0 & 1.794825 & 2.027785 & -0.39178 \\
\hline 70 & 6 & 0 & 3.578766 & 3.012750 & 0.22509 \\
\hline 71 & 6 & 0 & 3.109773 & 2.089288 & -2.080275 \\
\hline 72 & 1 & 0 & 6.175994 & -0.295067 & 1.314046 \\
\hline 73 & 1 & 0 & 4.998789 & 0.912757 & 1.898374 \\
\hline 74 & 1 & 0 & 4.54 & -0.800359 & 1.82391 \\
\hline 75 & 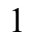 & 0 & 4.206543 & -1.902962 & -0.55224 \\
\hline 76 & 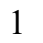 & 0 & 4.539528 & -0.899978 & -1.98915 \\
\hline 77 & 1 & ) & 5.877964 & -1.399421 & -0.91940 \\
\hline 78 & 1 & ) & 4.667771 & 2.986683 & 0.07529 \\
\hline 79 & 1 & ) & 3.222894 & 4.005553 & -0.086176 \\
\hline 80 & 1 & ) & 3.370316 & 2.888528 & 1.294979 \\
\hline 81 & 1 & ) & 4.173902 & 1.961792 & -2.32864 \\
\hline 82 & 1 & 0 & 2.534743 & 1.344125 & -2.64798 \\
\hline 83 & 1 & 0 & 2.806482 & 3.093096 & -2.41234 \\
\hline 84 & 3 & 0 & 1.609959 & -0.491921 & -0.61593 \\
\hline 85 & 8 & 0 & 1.079574 & -1.402152 & -2.23323 \\
\hline 86 & 6 & 0 & -0.293080 & -1.815327 & -2.20696 \\
\hline 87 & 6 & 0 & -0.263902 & -3.351137 & -2.10200 \\
\hline 88 & 6 & 0 & 1.188613 & -3.726993 & -2.47848 \\
\hline 89 & 6 & 0 & 1.779996 & -2.406446 & -2.97246 \\
\hline 90 & 1 & 0 & -0.751803 & -1.314543 & $-1.3475^{\prime}$ \\
\hline 91 & 1 & 0 & -0.778242 & -1.487456 & -3.14017 \\
\hline 92 & 1 & 0 & -0.504415 & -3.683111 & $-1.0847 t$ \\
\hline
\end{tabular}

S82 


$\begin{array}{rrrrrr}93 & 1 & 0 & -0.992809 & -3.801877 & -2.783874 \\ 94 & 1 & 0 & 1.739140 & -4.079339 & -1.598312 \\ 95 & 1 & 0 & 1.237501 & -4.506758 & -3.245279 \\ 96 & 1 & 0 & 2.849011 & -2.286340 & -2.775209 \\ 97 & 1 & 0 & 1.586113 & -2.257272 & -4.046108\end{array}$

\section{Thermodynamics:}

Zero-point correction $=$ Thermal correction to Energy= Thermal correction to Enthalpy= Thermal correction to Gibbs Free Energy= Sum of electronic and zero-point Energies= Sum of electronic and thermal Energies= Sum of electronic and thermal Enthalpies= Sum of electronic and thermal Free Energies=
0.872818 (Hartree/Particle) 0.918850

0.919794

0.792852

$-1507.281239$

$-1507.235207$

$-1507.234263$

$-1507.361205$ 
Structure 23 - M06-2x/jul-cc-pVDZ

Input:

$\# \mathrm{~m} 062 \mathrm{x} / \mathrm{jul}-\mathrm{cc}-\mathrm{pvDz}$ opt=(calcfc,noeigen,z-matrix $)$ optcyc $=50$ freq scrf $=(\mathrm{pcm}$,solvent=THF $)$

Optimized Structure:

\begin{tabular}{|c|c|c|c|c|}
\hline \multirow{3}{*}{$\begin{array}{l}\text { Center } \\
\text { Number } \\
-1\end{array}$} & \multirow{2}{*}{$\begin{array}{l}\text { Atomic } \\
\text { Number }\end{array}$} & \multirow{2}{*}{$\begin{array}{r}\text { Atomic } \\
\text { Type }\end{array}$} & \multicolumn{2}{|c|}{ Coordinates (Angstroms) } \\
\hline & & & $\mathrm{X}$ & $\mathrm{Z}$ \\
\hline & 7 & -0.049602 & -1.330405 & 1.602396 \\
\hline 2 & 8 & -0.012616 & -0.804063 & 0.275563 \\
\hline 3 & 6 & -0.436234 & -2.810221 & 1.486487 \\
\hline 4 & 6 & 1.350914 & -1.147237 & 2.179350 \\
\hline 5 & 6 & -1.068507 & -0.478158 & 2.409224 \\
\hline 6 & 6 & 1.196752 & 0.205493 & 2.872162 \\
\hline 7 & 6 & -0.206779 & 0.181363 & 3.493023 \\
\hline 8 & 1 & 3.146658 & 0.630178 & 1.330926 \\
\hline 9 & 1 & 1.423852 & -1.887444 & 2.991767 \\
\hline 10 & 1 & 1.250648 & 1.042884 & 2.150761 \\
\hline 11 & 1 & 1.996909 & 0.359906 & 3.613187 \\
\hline 12 & 1 & -0.569446 & 1.190342 & 3.753836 \\
\hline 13 & 1 & -0.153681 & -0.397242 & 4.438304 \\
\hline 14 & 1 & -1.769715 & -1.187341 & 2.868283 \\
\hline 15 & 6 & 0.576987 & -3.536627 & 0.597340 \\
\hline 16 & 6 & -0.460365 & -3.492612 & 2.859272 \\
\hline 17 & 6 & -1.815841 & -2.915934 & 0.836771 \\
\hline 18 & 1 & 1.581670 & -3.491515 & 1.029150 \\
\hline 19 & 1 & 0.275433 & -4.590142 & 0.514304 \\
\hline 20 & 1 & 0.596332 & -3.093894 & -0.403065 \\
\hline 21 & 1 & 0.548514 & -3.719728 & 3.218879 \\
\hline 22 & 1 & -0.973899 & -2.884916 & 3.611558 \\
\hline 23 & 1 & -1.001195 & -4.443400 & 2.759933 \\
\hline 24 & 1 & -2.609188 & -2.562195 & 1.504405 \\
\hline 25 & 1 & -1.837815 & -2.329203 & -0.085098 \\
\hline 26 & 1 & -2.014122 & -3.970078 & 0.599794 \\
\hline 27 & 3 & -1.466169 & 0.350804 & 0.521025 \\
\hline 28 & 8 & -3.286172 & -0.211739 & -0.317488 \\
\hline 29 & 6 & -4.369023 & -0.365819 & 0.619411 \\
\hline 30 & 6 & -5.452785 & -1.156600 & -0.114119 \\
\hline 31 & 6 & -4.643009 & -1.939489 & -1.152046 \\
\hline 32 & 6 & -3.575987 & -0.920474 & -1.528424 \\
\hline 33 & 1 & -3.980097 & -0.893997 & 1.498766 \\
\hline 34 & 1 & -4.706695 & 0.631593 & 0.932533 \\
\hline 35 & 1 & -6.023359 & -1.798255 & 0.565069 \\
\hline 36 & 1 & -6.151109 & -0.475187 & -0.616667 \\
\hline 37 & 1 & -4.178001 & -2.821782 & -0.694370 \\
\hline 38 & 1 & -5.238746 & -2.258209 & -2.013777 \\
\hline 39 & 1 & -2.640559 & -1.361960 & -1.893215 \\
\hline 40 & 1 & -3.953541 & -0.211255 & -2.282673 \\
\hline
\end{tabular}




\begin{tabular}{|c|c|c|c|c|c|}
\hline 41 & T & 0 & -2.008328 & 2.293668 & -0.157974 \\
\hline 42 & 1 & 0 & -2.969828 & 1.998142 & -0.000787 \\
\hline 43 & 6 & 0 & -1.858261 & 2.365827 & -1.625720 \\
\hline 44 & 6 & 0 & -1.791679 & 3.539455 & 0.606200 \\
\hline 45 & 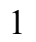 & & -1.962095 & 1.324663 & -1.971230 \\
\hline 46 & 6 & 0 & -2.959599 & 3.186801 & -2.298201 \\
\hline 47 & & 0 & -0.470835 & 2.845579 & -2.02618 \\
\hline 48 & . & 0 & -1.920348 & 4.416738 & -0.05034 \\
\hline 49 & 6 & ) & -2.841112 & 3.611601 & 1.710896 \\
\hline 50 & 6 & 0 & -0.387745 & 3.573651 & 1.202288 \\
\hline 51 & 1 & 0 & -3.949983 & 2.790673 & -2.03439 \\
\hline 52 & 1 & 0 & -2.915072 & 4.241807 & -1.99508 \\
\hline 53 & 1 & 0 & -2.855090 & 3.145867 & -3.39057 \\
\hline 54 & 1 & 0 & 0.300637 & 2.239838 & -1.53785 \\
\hline 55 & 1 & 0 & -0.346585 & 2.758069 & -3.11325 \\
\hline 56 & 1 & 0 & -0.315758 & 3.899123 & -1.75410 \\
\hline 57 & 1 & 0 & -3.853269 & 3.675808 & 1.287561 \\
\hline 58 & 1 & 0 & -2.781051 & 2.709819 & 2.338825 \\
\hline 59 & 1 & 0 & -2.677458 & 4.489075 & 2.34805 \\
\hline 60 & 1 & 0 & -0.239244 & 2.690935 & 1.84103 \\
\hline 61 & 1 & 0 & 0.381448 & 3.571017 & 0.42290 \\
\hline 62 & 1 & 0 & -0.253299 & 4.479564 & 1.80908 \\
\hline 63 & 7 & 0 & 3.343527 & 0.696043 & 0.33327 \\
\hline 64 & 6 & 0 & 4.790485 & 0.492396 & 0.123924 \\
\hline 65 & 6 & 0 & 2.784579 & 1.972143 & -0.145488 \\
\hline 66 & 1 & 0 & 5.264520 & 1.449440 & -0.148703 \\
\hline 67 & 6 & 0 & 5.432956 & 0.007968 & 1.41971 \\
\hline 68 & & 0 & 5.031315 & -0.503547 & -1.00823 \\
\hline 69 & 1 & 0 & 1.699605 & 1.878049 & 0.02156 \\
\hline 70 & 6 & 0 & 3.273453 & 3.179827 & 0.65598 \\
\hline 71 & 6 & 0 & 3.018880 & 2.150594 & -1.641284 \\
\hline 72 & 1 & 0 & 6.502849 & -0.187179 & 1.273536 \\
\hline 73 & 1 & 0 & 5.321765 & 0.757964 & 2.214771 \\
\hline 74 & 1 & 0 & 4.954361 & -0.924144 & 1.75288 \\
\hline 75 & 1 & ) & 4.629243 & -1.487437 & -0.72505 \\
\hline 76 & 1 & 0 & 4.525844 & -0.188275 & -1.92821 \\
\hline 77 & 1 & 0 & 6.106554 & -0.608390 & -1.20873 \\
\hline 78 & 1 & 0 & 4.361826 & 3.302715 & 0.56754 \\
\hline 79 & 1 & 0 & 2.800542 & 4.102912 & 0.29348 \\
\hline 80 & 1 & 0 & 3.021498 & 3.060435 & 1.719093 \\
\hline 81 & 1 & 0 & 4.087699 & 2.269453 & -1.865248 \\
\hline 82 & 1 & 0 & 2.641050 & 1.283512 & -2.19754 \\
\hline 83 & 1 & 0 & 2.502982 & 3.052650 & -1.99449 \\
\hline 84 & 3 & 0 & 1.884204 & -0.824223 & 0.11382 \\
\hline 85 & 8 & 0 & 1.968719 & -1.210878 & -1.86907 \\
\hline 86 & 6 & 0 & 0.810431 & -0.745333 & -2.56537 \\
\hline 87 & 6 & 0 & 0.029970 & -2.010438 & -2.890856 \\
\hline 88 & 6 & 0 & 1.146444 & -3.028622 & -3.183509 \\
\hline 89 & 6 & 0 & 2.381308 & -2.447269 & -2.46247 \\
\hline 90 & 1 & 0 & 0.278900 & -0.062469 & -1.89326 \\
\hline 91 & 1 & 0 & 1.120316 & -0.213731 & -3.48158 \\
\hline 92 & 1 & 0 & -0.545450 & -2.302975 & -2.00491 \\
\hline 93 & 1 & 0 & -0.654108 & -1.877429 & -3.7365 \\
\hline
\end{tabular}




$\begin{array}{llllll}94 & 1 & 0 & 0.895528 & -4.028799 & -2.813888 \\ 95 & 1 & 0 & 1.333173 & -3.105273 & -4.260490 \\ 96 & 1 & 0 & 2.749441 & -3.091339 & -1.654801 \\ 97 & 1 & 0 & 3.207008 & -2.255344 & -3.163029\end{array}$

\section{Thermodynamics:}

Zero-point correction $=$

Thermal correction to Energy=

Thermal correction to Enthalpy=

Thermal correction to Gibbs Free Energy=

Sum of electronic and zero-point Energies=

Sum of electronic and thermal Energies=

Sum of electronic and thermal Enthalpies=

Sum of electronic and thermal Free Energies=
0.878860 (Hartree/Particle)

0.925108

0.926052

0.800065

$-1507.284045$

$-1507.237797$

$-1507.236852$

$-1507.362840$ 
Structure 23 $3_{\text {TS }}$ - M06-2x/jul-cc-pVDZ

Input:

$\#$ m062x/jul-cc-pvDz opt=(calcfc,noeigen,z-matrix,ts) optcyc=50 freq scr $\mathrm{f}=(\mathrm{pcm}$, solvent $=\mathrm{THF})$

Optimized Structure:

\begin{tabular}{|c|c|c|c|c|}
\hline \multirow{3}{*}{$\begin{array}{l}\text { Center } \\
\text { Number } \\
1\end{array}$} & \multirow{2}{*}{$\begin{array}{l}\text { Atomic } \\
\text { Number }\end{array}$} & \multirow{2}{*}{$\begin{array}{r}\text { Atomic } \\
\text { Type }\end{array}$} & \multicolumn{2}{|c|}{ Coordinates (Angstroms } \\
\hline & & & $\mathrm{X}$ & $\mathrm{Z}$ \\
\hline & 7 & 0.019122 & -1.191060 & -1.976805 \\
\hline 2 & 8 & -0.025724 & -0.731294 & -0.239885 \\
\hline 3 & 6 & 0.333123 & -2.668863 & -1.824014 \\
\hline 4 & 6 & -1.179838 & -0.876840 & -2.580832 \\
\hline 5 & 6 & 1.095288 & -0.316062 & -2.534989 \\
\hline 6 & 6 & -1.079186 & 0.549757 & -3.036892 \\
\hline 7 & 6 & 0.362988 & 0.560213 & -3.558090 \\
\hline 8 & 1 & -2.947953 & 0.570507 & -1.346585 \\
\hline 9 & 1 & -2.078445 & -1.269846 & -2.103448 \\
\hline 10 & 1 & -1.154148 & 1.284213 & -2.202122 \\
\hline 11 & 1 & -1.826025 & 0.824121 & -3.795889 \\
\hline 12 & 1 & 0.775596 & 1.579418 & -3.621803 \\
\hline 13 & 1 & 0.364513 & 0.131316 & -4.580144 \\
\hline 14 & 1 & 1.837251 & -0.971059 & -3.007914 \\
\hline 15 & 6 & -0.754390 & -3.368767 & -1.008922 \\
\hline 16 & 6 & 0.393716 & -3.308372 & -3.217037 \\
\hline 17 & 6 & 1.672512 & -2.859445 & -1.116102 \\
\hline 18 & 1 & -1.750399 & -3.204760 & -1.437150 \\
\hline 19 & 1 & -0.560031 & -4.449823 & -1.030450 \\
\hline 20 & 1 & -0.729478 & -3.017007 & 0.026578 \\
\hline 21 & 1 & -0.605633 & -3.375233 & -3.657113 \\
\hline 22 & 1 & 1.034266 & -2.728859 & -3.891468 \\
\hline 23 & 1 & 0.813565 & -4.319436 & -3.121870 \\
\hline 24 & 1 & 2.511707 & -2.526282 & -1.737367 \\
\hline 25 & 1 & 1.661859 & -2.297336 & -0.180415 \\
\hline 26 & 1 & 1.808731 & -3.930500 & -0.910413 \\
\hline 27 & 3 & 1.391167 & 0.332268 & -0.554106 \\
\hline 28 & 8 & 3.252927 & -0.258092 & 0.245340 \\
\hline 29 & 6 & 4.348982 & -0.427972 & -0.670519 \\
\hline 30 & 6 & 5.362736 & -1.314670 & 0.050545 \\
\hline 31 & 6 & 4.465666 & -2.138285 & 0.979374 \\
\hline 32 & 6 & 3.425681 & -1.103308 & 1.389998 \\
\hline 33 & 1 & 3.959657 & -0.894871 & -1.584628 \\
\hline 34 & 1 & 4.746545 & 0.563708 & -0.924604 \\
\hline 35 & 1 & 5.945014 & -1.926241 & -0.646405 \\
\hline 36 & 1 & 6.057033 & -0.702000 & 0.640251 \\
\hline 37 & 1 & 3.986980 & -2.955978 & 0.426125 \\
\hline 38 & 1 & 5.000240 & -2.555910 & 1.839062 \\
\hline 39 & 1 & 2.445959 & -1.527031 & $1.6455 \mathrm{c}$ \\
\hline
\end{tabular}




\begin{tabular}{|c|c|c|c|c|c|}
\hline 40 & 1 & 0 & 3.784657 & -0.494225 & 5573 \\
\hline 41 & 7 & 0 & 1.944718 & 2.247583 & 0.284515 \\
\hline 42 & 1 & 0 & 2.888563 & 1.943480 & 0.053409 \\
\hline 43 & 6 & & 1.845988 & 2.120712 & 1.752841 \\
\hline 44 & 6 & & 1.756857 & 3.594021 & -0.28976 \\
\hline 45 & 1 & () & 1.915915 & 1.039843 & 1.94703 \\
\hline 46 & 6 & 0 & 3.006107 & 2.794736 & 2.48775 \\
\hline 47 & 6 & 0 & 0.496150 & 2.588000 & 2.27796 \\
\hline 48 & 1 & 0 & 1.972756 & 4.367246 & 0.467784 \\
\hline 49 & 6 & 0 & 2.744337 & 3.764475 & -1.439710 \\
\hline 50 & 6 & 0 & 0.327672 & 3.786860 & -0.788379 \\
\hline 51 & 1 & ) & 3.966916 & 2.402608 & 2.12597 \\
\hline 52 & 1 & 0 & 2.997692 & 3.883586 & 2.34196 \\
\hline 53 & 1 & 0 & 2.941744 & 2.599479 & 3.566644 \\
\hline 54 & 1 & 0 & -0.319633 & 2.068052 & 1.76134 \\
\hline 55 & 1 & 0 & 0.422827 & 2.366887 & 3.350664 \\
\hline 56 & 1 & 0 & 0.366557 & 3.671739 & 2.150305 \\
\hline 57 & 1 & 0 & 3.781004 & 3.710487 & -1.079172 \\
\hline 58 & 1 & 0 & 2.589684 & 2.965907 & $-2.18038 c$ \\
\hline 59 & 1 & 0 & 2.601723 & 4.731503 & -1.937192 \\
\hline 60 & 1 & 0 & 0.088209 & 3.009321 & -1.52900 \\
\hline 61 & 1 & 0 & -0.398464 & 3.720377 & 0.028792 \\
\hline 62 & 1 & 0 & 0.219238 & 4.773173 & -1.26015 \\
\hline 63 & 7 & 0 & -3.202187 & 0.627885 & -0.361479 \\
\hline 64 & 6 & 0 & -4.651662 & 0.365970 & -0.238186 \\
\hline 65 & 6 & 0 & -2.732646 & 1.951526 & 0.103419 \\
\hline 66 & 1 & 0 & -5.185909 & 1.312837 & -0.06058 \\
\hline 67 & 6 & 0 & -5.168172 & -0.229832 & -1.54303 \\
\hline 68 & 6 & 0 & -4.924398 & -0.571167 & 0.93487 \\
\hline 69 & 1 & 0 & -1.638630 & 1.907201 & -0.01407 \\
\hline 70 & 6 & 0 & -3.246627 & 3.101246 & -0.76347 \\
\hline 71 & 6 & 0 & -3.045169 & 2.168690 & 1.57944 \\
\hline 72 & 1 & 0 & -6.233222 & -0.480000 & -1.46246 \\
\hline 73 & 1 & 0 & -5.038064 & 0.475824 & -2.37479 \\
\hline 74 & 1 & 0 & -4.616966 & -1.151500 & -1.78208 \\
\hline 75 & 1 & 0 & -4.489834 & -1.560410 & 0.72594 \\
\hline 76 & 1 & 0 & -4.474790 & -0.191834 & 1.86010 \\
\hline 77 & 1 & 0 & -6.004672 & -0.694724 & 1.08980 \\
\hline 78 & 1 & 0 & -4.339973 & 3.191469 & -0.70537 \\
\hline 79 & 1 & 0 & -2.813744 & 4.054111 & -0.43075 \\
\hline 80 & 1 & 0 & -2.963461 & 2.946137 & -1.81377 \\
\hline 81 & 1 & 0 & -4.127604 & 2.248800 & 1.75055 \\
\hline 82 & 1 & 0 & -2.653534 & 1.343931 & 2.18936 \\
\hline 83 & 1 & 0 & -2.584610 & 3.106032 & 1.91806 \\
\hline 84 & 3 & 0 & -1.716368 & -0.642811 & 0.31100 \\
\hline 85 & 8 & 0 & -1.852382 & -1.077486 & 2.23472 \\
\hline 86 & 6 & 0 & -0.533578 & -0.902090 & $2.7947 \mathrm{~S}$ \\
\hline 87 & 6 & 0 & -0.061098 & -2.308022 & 3.12769 \\
\hline 88 & 6 & 0 & -1.362902 & -2.968739 & 3.58824 \\
\hline 89 & 6 & 0 & -2.393844 & -2.349263 & 2.64434 \\
\hline 90 & 1 & 0 & 0.075688 & -0.411726 & 2.02483 \\
\hline 91 & 1 & 0 & -0.613233 & -0.276967 & 3.69810 \\
\hline 92 & 1 & 0 & 0.319674 & -2.792276 & 2.21745 \\
\hline
\end{tabular}




$\begin{array}{rrrrrr}93 & 1 & 0 & 0.722910 & -2.318355 & 3.893060 \\ 94 & 1 & 0 & -1.347520 & -4.061321 & 3.517162 \\ 95 & 1 & 0 & -1.580369 & -2.691501 & 4.627646 \\ 96 & 1 & 0 & -2.540892 & -2.967332 & 1.746857 \\ 97 & 1 & 0 & -3.367725 & -2.180270 & 3.119708\end{array}$

\section{Thermodynamics:}

Zero-point correction $=$

Thermal correction to Energy=

Thermal correction to Enthalpy=

Thermal correction to Gibbs Free Energy=

Sum of electronic and zero-point Energies=

Sum of electronic and thermal Energies=

Sum of electronic and thermal Enthalpies=

Sum of electronic and thermal Free Energies=
0.877113 (Hartree/Particle)

0.923241

0.924185

0.798707

$-1507.260854$

$-1507.214726$

$-1507.213782$

$-1507.339260$ 


\section{Structure 28 - M06-2x/jul-cc-pVDZ}

Input:

$\# \mathrm{~m} 062 \mathrm{x} / \mathrm{jul}$-cc-pVDZ opt=(calcfc,noeigen $)$ optcyc $=50$ freq $\mathrm{scrf}=(\mathrm{pcm}$, solve $\mathrm{nt}=\mathrm{THF}, \mathrm{read})$

Optimized Structure:

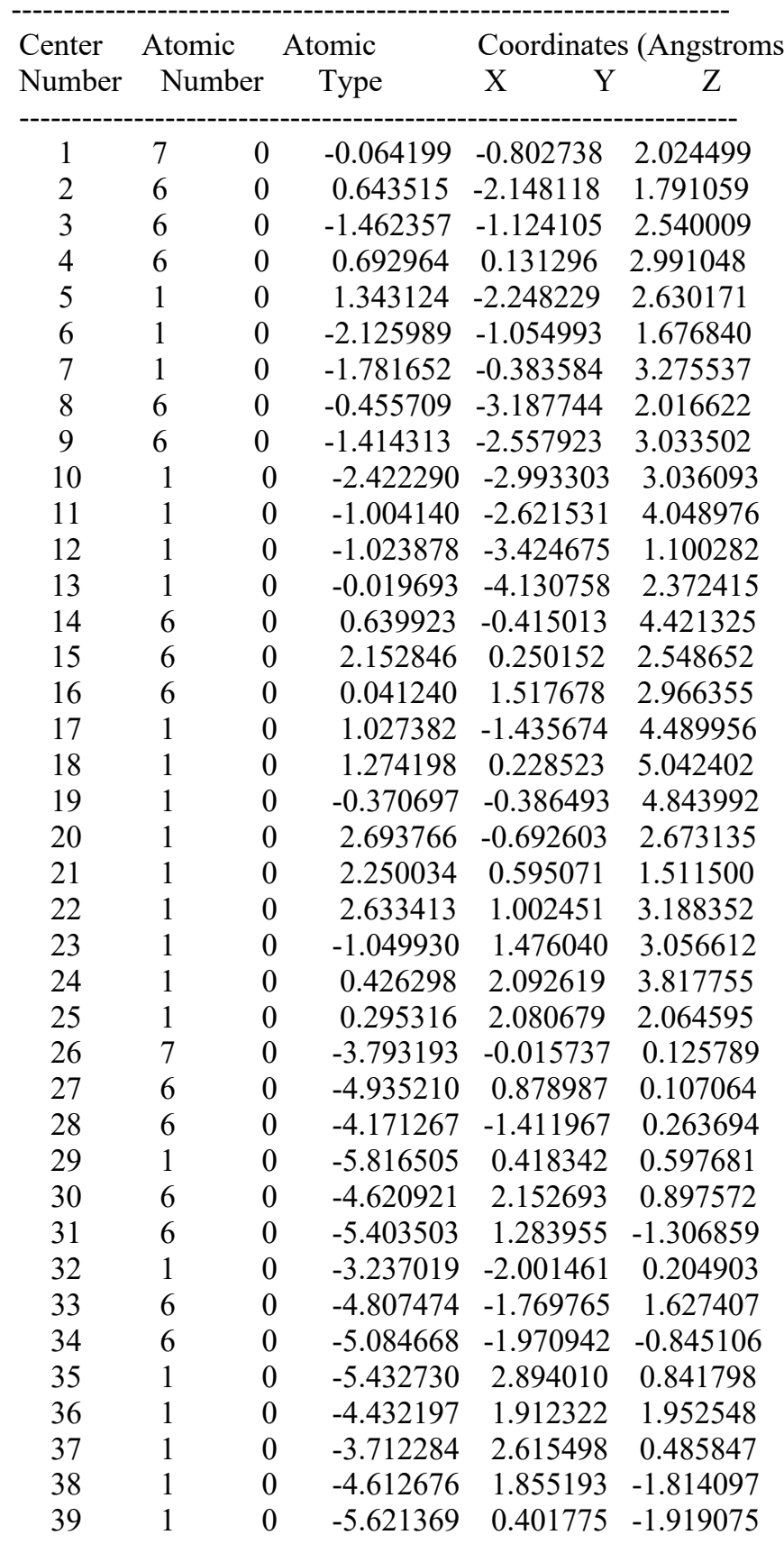




\begin{tabular}{|c|c|c|c|c|c|}
\hline 40 & 1 & 0 & -6.307890 & 1.912931 & -1.269897 \\
\hline 41 & 1 & 0 & -5.813647 & -1.337390 & 1.721959 \\
\hline 42 & 1 & 0 & -4.906404 & -2.860061 & 1.745684 \\
\hline 43 & 1 & 0 & -4.196480 & -1.382986 & 2.453850 \\
\hline 44 & 1 & 0 & -6.075958 & -1.496278 & -0.807774 \\
\hline 45 & 1 & 0 & -4.652371 & -1.783097 & -1.83766 \\
\hline 46 & 1 & 0 & -5.231641 & -3.055833 & -0.72643 \\
\hline 47 & 3 & 0 & -1.972847 & 0.538270 & 0.22466 \\
\hline 48 & 8 & 0 & -0.197560 & -0.124895 & 0.78792 \\
\hline 49 & 3 & ) & 1.206873 & -1.429279 & -0.099670 \\
\hline 50 & 7 & 0 & 0.398879 & -2.753451 & -1.496256 \\
\hline 51 & 1 & 0 & 0.523777 & -3.478222 & -0.787566 \\
\hline 52 & 6 & 0 & -1.050304 & -2.456951 & -1.52118 \\
\hline 53 & 6 & 0 & 0.977309 & -3.249454 & -2.76534 \\
\hline 54 & ( & 0 & -1.380141 & -1.342934 & -2.50407 \\
\hline 55 & ( & 0 & -1.915316 & -3.695529 & -1.75540 \\
\hline 56 & . & 0 & -1.269237 & -2.071177 & -0.51686 \\
\hline 57 & 6 & 0 & 1.746089 & -2.146401 & -3.484037 \\
\hline 58 & 6 & 0 & 1.889848 & -4.435581 & -2.478607 \\
\hline 59 & 1 & 0 & 0.167723 & -3.595932 & -3.42717 \\
\hline 60 & 1 & 0 & -0.749915 & -0.462509 & -2.3145 \\
\hline 61 & 1 & 0 & -1.229916 & -1.659632 & -3.54573 \\
\hline 62 & 1 & 0 & -2.429612 & -1.049924 & -2.37353 \\
\hline 63 & 1 & 0 & -1.767329 & -4.110881 & -2.76121 \\
\hline 64 & 1 & 0 & -1.678596 & -4.477688 & -1.02056 \\
\hline 65 & & 0 & -2.976651 & -3.439233 & -1.652006 \\
\hline 66 & 1 & 0 & 1.109178 & -1.276968 & -3.679200 \\
\hline 67 & 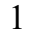 & ) & 2.592185 & -1.821597 & -2.86253 \\
\hline 68 & 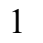 & 0 & 2.130888 & -2.515896 & -4.44438 \\
\hline 69 & & 0 & 2.699804 & -4.126555 & -1.80281 \\
\hline 70 & 1 & 0 & 1.334134 & -5.259288 & -2.01045 \\
\hline 71 & 1 & 0 & 2.342633 & -4.807453 & -3.40585 \\
\hline 72 & 3 & 0 & 0.623113 & 1.438415 & 0.004320 \\
\hline 73 & 7 & 0 & 2.132519 & 2.524794 & -0.395940 \\
\hline 74 & 6 & 0 & 2.490259 & 2.360474 & -1.79116 \\
\hline 75 & 6 & 0 & 3.043860 & 3.429746 & 0.27855 \\
\hline 76 & ( & 0 & 1.760363 & 1.152584 & -2.37784 \\
\hline 77 & 6 & 0 & 2.173085 & 3.580962 & -2.67755 \\
\hline 78 & 1 & 0 & 3.579985 & 2.157911 & -1.92019 \\
\hline 79 & 6 & ) & 2.441250 & 3.976220 & 1.572113 \\
\hline 80 & 6 & 0 & 4.416332 & 2.811169 & 0.619425 \\
\hline 81 & 1 & 0 & 3.264115 & 4.317284 & -0.353926 \\
\hline 82 & 1 & 0 & 2.030273 & 0.221256 & -1.86269 \\
\hline 83 & 1 & 0 & 0.671520 & 1.294065 & -2.29183 \\
\hline 84 & 1 & 0 & 1.981911 & 1.015085 & -3.44516 \\
\hline 85 & 1 & 0 & 1.082292 & 3.720312 & -2.73216 \\
\hline 86 & 1 & 0 & 2.613371 & 4.500256 & -2.27206 \\
\hline 87 & 1 & 0 & 2.556257 & 3.446408 & -3.70088 \\
\hline 88 & 1 & 0 & 2.214237 & 3.152948 & 2.262776 \\
\hline 89 & 1 & 0 & 3.145653 & 4.652987 & 2.07685 \\
\hline 90 & 1 & 0 & 1.516192 & 4.534742 & 1.37696 \\
\hline 91 & 1 & 0 & 4.901520 & 2.383312 & -0.26852 \\
\hline 92 & 1 & 0 & 5.097448 & 3.566477 & 1.04074 \\
\hline
\end{tabular}




$\begin{array}{cccccc}93 & 1 & 0 & 4.289608 & 2.007782 & 1.360796 \\ 94 & 8 & 0 & -1.225808 & 2.233859 & -0.577281 \\ 95 & 6 & 0 & -1.141629 & 3.514358 & 0.110627 \\ 96 & 6 & 0 & -1.514975 & 4.567969 & -0.924038 \\ 97 & 1 & 0 & -0.111961 & 3.629342 & 0.463045 \\ 98 & 1 & 0 & -1.838463 & 3.490767 & 0.957549 \\ 99 & 6 & 0 & -2.444193 & 3.800975 & -1.867685 \\ 100 & 1 & 0 & -0.615598 & 4.902194 & -1.456373 \\ 101 & 1 & 0 & -1.992757 & 5.438266 & -0.462833 \\ 102 & 6 & 0 & -1.796397 & 2.425488 & -1.898610 \\ 103 & 1 & 0 & -3.457438 & 3.744561 & -1.452240 \\ 104 & 1 & 0 & -2.500163 & 4.242652 & -2.868002 \\ 105 & 1 & 0 & -2.494370 & 1.598652 & -2.072382 \\ 106 & 1 & 0 & -0.986511 & 2.391467 & -2.638684 \\ 107 & 8 & 0 & 3.171236 & -1.507915 & -0.267086 \\ 108 & 6 & 0 & 3.806341 & -2.529914 & 0.524851 \\ 109 & 6 & 0 & 5.297566 & -2.226774 & 0.458249 \\ 110 & 1 & 0 & 3.530967 & -3.507341 & 0.112308 \\ 111 & 1 & 0 & 3.423146 & -2.465447 & 1.551472 \\ 112 & 6 & 0 & 5.288590 & -0.698390 & 0.395489 \\ 113 & 1 & 0 & 5.735272 & -2.643874 & -0.458292 \\ 114 & 1 & 0 & 5.838958 & -2.628510 & 1.321215 \\ 115 & 6 & 0 & 4.104867 & -0.435769 & -0.524197 \\ 116 & 1 & 0 & 5.094860 & -0.277099 & 1.391777 \\ 117 & 1 & 0 & 6.218178 & -0.267355 & 0.008864 \\ 118 & 1 & 0 & 3.592401 & 0.513879 & -0.332881 \\ 119 & 1 & 0 & 4.404327 & -0.485825 & -1.583291 \\ & & & & & \end{array}$

\section{Thermodynamics:}

Zero-point correction $=$ Thermal correction to Energy= Thermal correction to Enthalpy= Thermal correction to Gibbs Free Energy= Sum of electronic and zero-point Energies= Sum of electronic and thermal Energies= Sum of electronic and thermal Enthalpies= Sum of electronic and thermal Free Energies=
1.076677 (Hartree/Particle)

1.133324

1.134269

0.989752

$-1806.389985$

$-1806.333338$

$-1806.332394$

$-1806.476910$ 


\section{Models with Additional LDA Coordination}

\section{Structure S2}

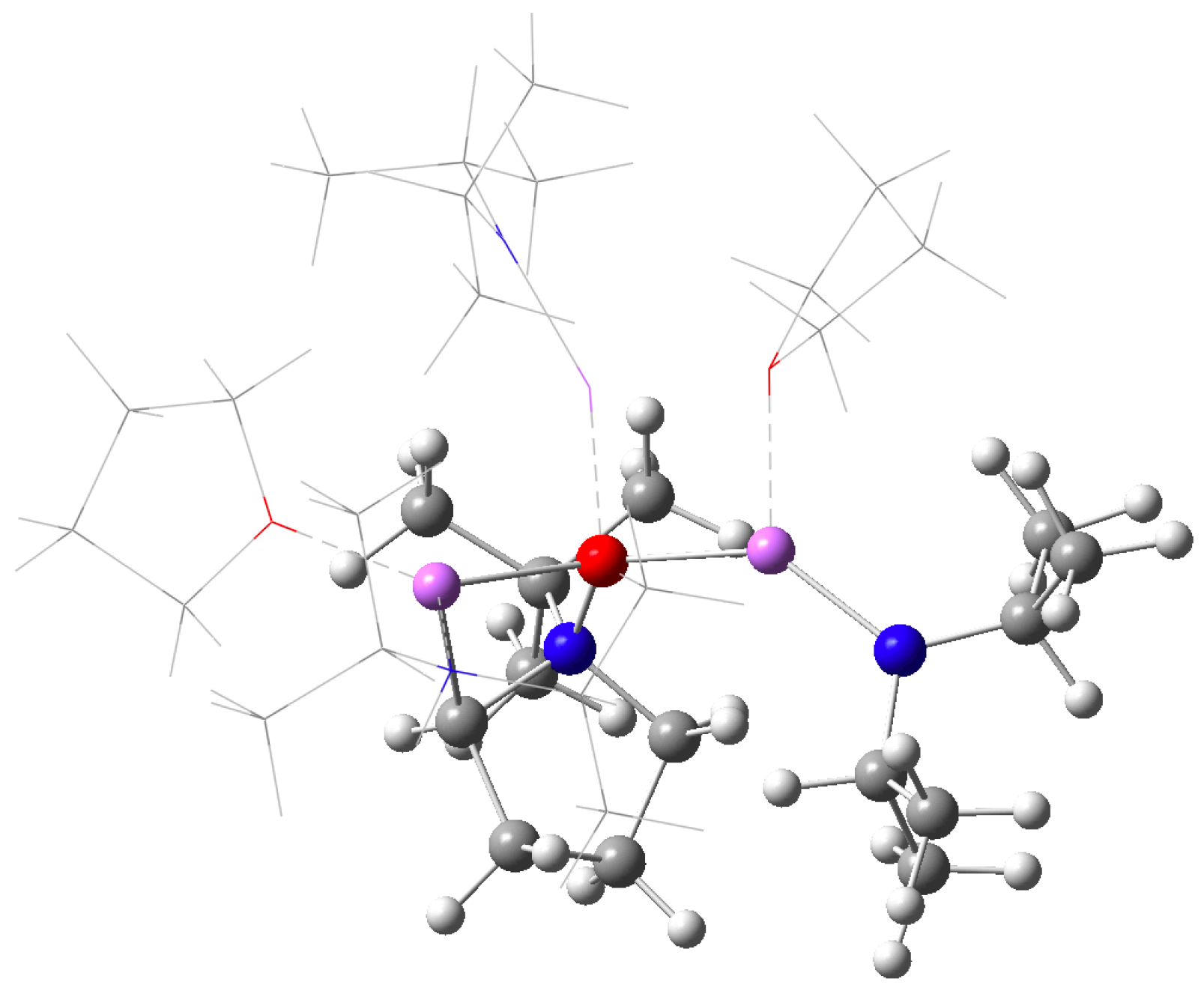

Figure S22. Ball and Stick Model for S2, additional LDA stabilization of 22. Explicit solvent depicted in a wireframe model. Color denotes chemical composition; grey=car- bon, white=hydrogen, blue=nitrogen, red=oxygen, and purple=lithium . 


\section{Structure S2 - M06-2x/jul-cc-pVDZ}

Input:

$\#$ m062x/jul-cc-pvDz opt=(calcfc,noeigen $)$ optcyc $=50$ freq $\mathrm{scrf}=(\mathrm{pcm}$, solve $\mathrm{nt}=\mathrm{THF}$,read)

Optimized Structure:

\begin{tabular}{|c|c|c|c|c|}
\hline \multirow{2}{*}{$\begin{array}{l}\text { Center } \\
\text { Number }\end{array}$} & Atomic & \multirow{2}{*}{$\begin{array}{l}\text { Atomic } \\
\text { Type }\end{array}$} & \multicolumn{2}{|c|}{ Coordinates (Angstroms } \\
\hline & Numbe & & $\mathrm{X}$ & Z \\
\hline 1 & 7 & 1.270189 & 2.671382 & -1.219643 \\
\hline 2 & 8 & -0.119220 & 0.989548 & -0.198007 \\
\hline 3 & 6 & 2.035855 & 3.163103 & -0.065838 \\
\hline 4 & 6 & 1.969855 & 1.763109 & -2.141892 \\
\hline 5 & 6 & -0.070401 & 2.104479 & -1.009545 \\
\hline 6 & 6 & 0.843535 & 1.111293 & -2.976733 \\
\hline 7 & 6 & -0.442812 & 1.772946 & -2.460383 \\
\hline 8 & 1 & 2.549818 & 0.981985 & -1.619133 \\
\hline 9 & 1 & 2.664504 & 2.333919 & -2.773317 \\
\hline 10 & 1 & 0.818614 & 0.032211 & -2.785555 \\
\hline 11 & 1 & 0.989123 & 1.256170 & -4.053591 \\
\hline 12 & 1 & -1.320134 & 1.115678 & -2.507927 \\
\hline 13 & 1 & -0.663913 & 2.696406 & -3.012262 \\
\hline 14 & 1 & -0.719080 & 2.912771 & -0.616621 \\
\hline 15 & 6 & 2.390613 & 2.088103 & 0.983090 \\
\hline 16 & 6 & 3.344120 & 3.765654 & -0.593223 \\
\hline 17 & 6 & 1.232722 & 4.270833 & 0.621015 \\
\hline 18 & 1 & 2.823773 & 1.197896 & 0.507778 \\
\hline 19 & 1 & 3.136845 & 2.484367 & 1.687281 \\
\hline 20 & 1 & 1.500546 & 1.798651 & 1.552693 \\
\hline 21 & 1 & 4.018348 & 2.985930 & -0.970710 \\
\hline 22 & 1 & 3.136786 & 4.474965 & -1.405474 \\
\hline 23 & 1 & 3.862565 & 4.296757 & 0.215867 \\
\hline 24 & 1 & 0.988282 & 5.066936 & -0.094605 \\
\hline 25 & 1 & 0.298954 & 3.882565 & 1.047316 \\
\hline 26 & 1 & 1.821663 & 4.702901 & 1.441248 \\
\hline 27 & 3 & -1.511304 & -0.057752 & $2 \quad 0.290442$ \\
\hline 28 & 8 & -2.915608 & 1.276105 & 0.906238 \\
\hline 29 & 6 & -3.752824 & 1.739957 & -0.164650 \\
\hline 30 & 6 & -4.343472 & 3.043728 & 0.344624 \\
\hline 31 & 6 & -3.140588 & 3.654041 & 1.068776 \\
\hline 32 & 6 & -2.407700 & 2.425782 & 1.621764 \\
\hline 33 & 1 & -3.139372 & 1.905594 & -1.065783 \\
\hline 34 & 1 & -4.502104 & 0.967315 & -0.370054 \\
\hline 35 & 1 & -4.726412 & 3.674563 & -0.464080 \\
\hline 36 & 1 & -5.159504 & 2.833222 & 1.047561 \\
\hline 37 & 1 & -2.501739 & 4.188458 & 0.354396 \\
\hline 38 & 1 & -3.425907 & 4.352254 & 1.861999 \\
\hline 39 & 1 & -1.325985 & 2.480939 & 1.454433 \\
\hline
\end{tabular}




\begin{tabular}{|c|c|c|c|c|c|}
\hline 40 & 1 & 0 & -2.610249 & 2.259839 & 2.687010 \\
\hline 41 & 7 & 0 & -2.824337 & -1.549099 & -0.417893 \\
\hline 42 & 1 & 0 & -3.678236 & -1.057276 & -0.154536 \\
\hline 43 & 6 & 0 & -2.706443 & -2.671249 & 0.548590 \\
\hline 44 & 6 & 0 & -2.979262 & -1.941495 & -1.842547 \\
\hline 45 & . & 0 & -2.475583 & -2.193681 & 1.51451 \\
\hline 46 & 6 & 0 & -4.010389 & -3.451524 & 0.71645 \\
\hline 47 & 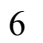 & 0 & -1.546419 & -3.587459 & 0.18804 \\
\hline 48 & . & 0 & -3.425714 & -2.947340 & $-1.9010 s$ \\
\hline 49 & 6 & 0 & -3.933485 & -0.968770 & -2.52635 \\
\hline 50 & 6 & 0 & -1.637930 & -1.956554 & -2.57136 \\
\hline 51 & 1 & 0 & -4.829955 & -2.783354 & 1.01292 \\
\hline 52 & 1 & 0 & -4.295960 & -3.963613 & -0.21206 \\
\hline 53 & . & 0 & -3.894486 & -4.214294 & 1.49735 \\
\hline 54 & . & 0 & -0.626108 & -3.012714 & 0.02569 \\
\hline 55 & 1 & 0 & -1.372401 & -4.302368 & 1.00187 \\
\hline 56 & 1 & 0 & -1.765775 & -4.166073 & $-0.7191<$ \\
\hline 57 & 1 & 0 & -4.912215 & -0.951785 & $-2.0265 \mathrm{~S}$ \\
\hline 58 & 1 & ) & -3.515954 & 0.047843 & -2.52022 \\
\hline 59 & 1 & 0 & -4.089654 & -1.264910 & -3.57027 \\
\hline 60 & 1 & 0 & -1.122168 & -0.999166 & -2.42156 \\
\hline 61 & 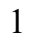 & 0 & -0.977887 & -2.756260 & -2.22321 \\
\hline 62 & 1 & 0 & -1.802321 & -2.099783 & -3.6477 \\
\hline 63 & ? & ) & 2.485083 & -1.463232 & $-0.1869 c$ \\
\hline 64 & 6 & 0 & 3.869834 & -1.685538 & 0.15850 \\
\hline 65 & 6 & 0 & 2.012143 & -2.262637 & -1.29929 \\
\hline 66 & 1 & 0 & 4.218557 & -2.686126 & -0.17562 \\
\hline 67 & 6 & 0 & 4.854261 & -0.667719 & -0.46151 \\
\hline 68 & ( & 0 & 4.038765 & -1.657899 & 1.68262 \\
\hline 69 & 1 & ) & 0.973028 & -1.931660 & -1.49225 \\
\hline 70 & 6 & 0 & 2.765990 & -2.078722 & -2.63341 \\
\hline 71 & 6 & 0 & 1.925094 & -3.778722 & -1.00856 \\
\hline 72 & 1 & ) & 5.902692 & -0.936623 & -0.250462 \\
\hline 73 & 1 & 0 & 4.723505 & -0.607927 & -1.54826 \\
\hline 74 & 1 & ) & 4.67 & 0.334870 & -0.04531 \\
\hline 75 & 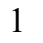 & 0 & 3.620265 & -0.717008 & 2.07604 \\
\hline 76 & 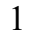 & 0 & 3.489084 & -2.491703 & 2.13972 \\
\hline 77 & 1 & 0 & 5.094532 & -1.718659 & 1.98980 \\
\hline 78 & 1 & 0 & 3.788633 & -2.478045 & -2.55528 \\
\hline 79 & 1 & ) & 2.260041 & -2.613710 & -3.45283 \\
\hline 80 & 1 & 0 & 2.833566 & -1.014751 & -2.89543 \\
\hline 81 & 1 & 0 & 2.928673 & -4.218306 & -0.91224 \\
\hline 82 & 1 & 0 & 1.385223 & -3.959089 & -0.06985 \\
\hline 83 & 1 & 0 & 1.407279 & -4.316074 & -1.81953 \\
\hline 84 & 3 & 0 & 1.162584 & -0.215297 & 0.32557 \\
\hline 85 & 8 & 0 & -0.163280 & -0.872702 & 1.85299 \\
\hline 86 & 6 & 0 & -0.286475 & 0.084831 & 2.91658 \\
\hline 87 & 6 & 0 & 0.904796 & -0.205016 & 3.81296 \\
\hline 88 & 6 & 0 & 0.955023 & -1.738775 & 3.78785 \\
\hline 89 & 6 & 0 & 0.311855 & -2.110129 & 2.43871 \\
\hline 90 & 1 & 0 & -0.278534 & 1.075666 & 2.45657 \\
\hline 91 & 1 & 0 & -1.240892 & -0.073824 & 3.44559 \\
\hline 92 & 1 & 0 & 1.810715 & 0.223216 & 3.36425 \\
\hline
\end{tabular}




$\begin{array}{rrrrrr}93 & 1 & 0 & 0.783579 & 0.205307 & 4.820895 \\ 94 & 1 & 0 & 1.978454 & -2.118852 & 3.866055 \\ 95 & 1 & 0 & 0.371396 & -2.154388 & 4.617332 \\ 96 & 1 & 0 & 1.025477 & -2.525356 & 1.718202 \\ 97 & 1 & 0 & -0.541510 & -2.786207 & 2.575702\end{array}$

\section{Thermodynamics:}

Zero-point correction $=$

Thermal correction to Energy=

Thermal correction to Enthalpy=

Thermal correction to Gibbs Free Energy=

Sum of electronic and zero-point Energies=

Sum of electronic and thermal Energies=

Sum of electronic and thermal Enthalpies=

Sum of electronic and thermal Free Energies=
0.879643 (Hartree/Particle)

0.925798

0.926742

0.801344

$-1507.411522$

$-1507.365368$

$-1507.364423$

$-1507.489821$ 


\section{Structure S3 $3_{\mathrm{TS}}$}

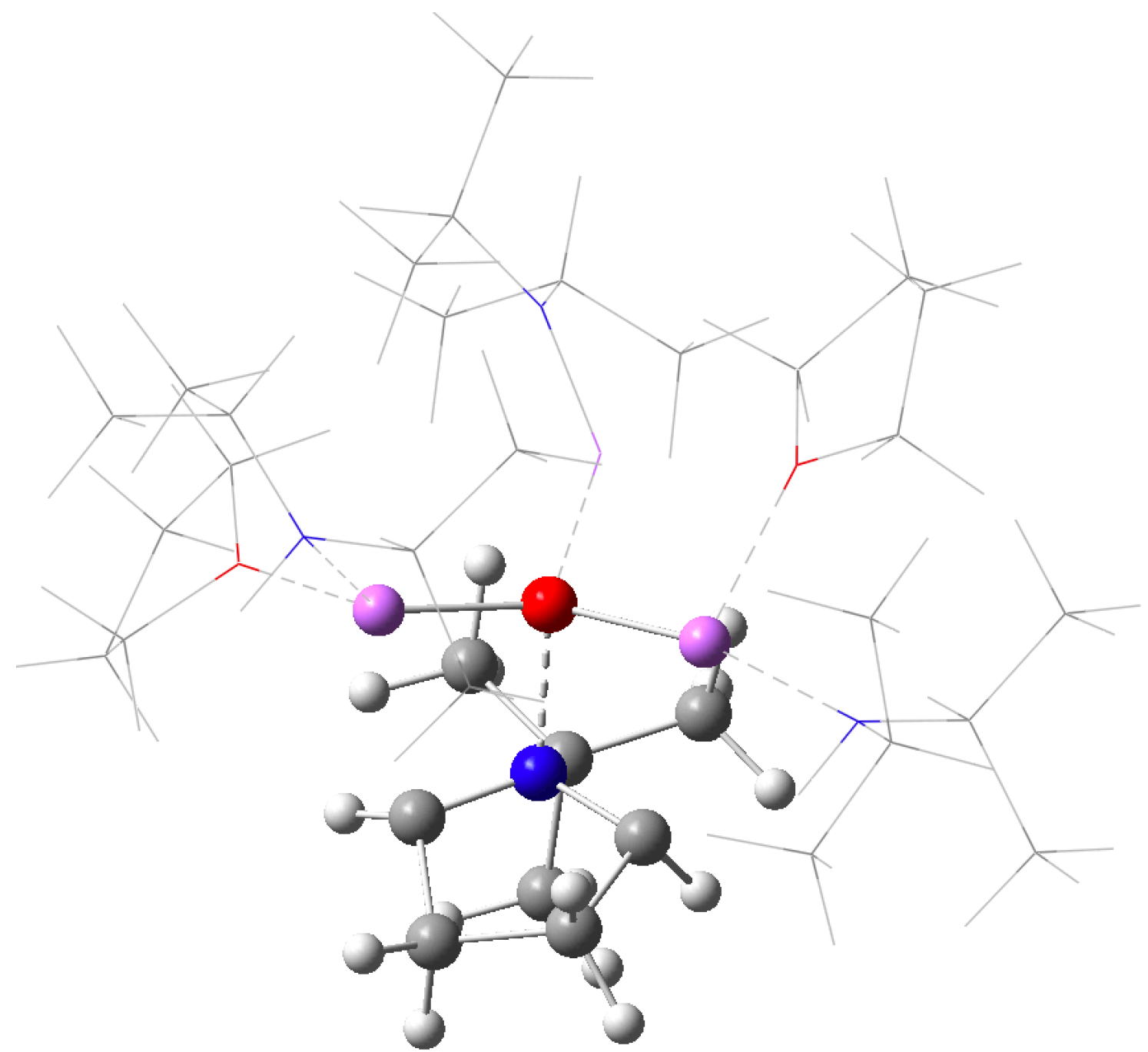

Figure S23. Ball and Stick Model for $\mathbf{S} 3_{\mathrm{TS}}$, additional LDA stabilization of $\mathbf{2 3}_{\mathrm{TS}}$. Explicit solvent depicted in a wireframe model. Color denotes chemical composition; grey=car- bon, white=hydrogen, blue=nitrogen, red=oxygen, and purple=lithium . 


\section{Structure S3 ${ }_{\text {TS }}$ - M06-2x/jul-cc-pVDZ}

Input:

$\#$ 062x/jul-cc-pVDZ opt=(calcfc,noeigen,ModRedun,ts) optcyc=50 freq scr $\mathrm{f}=(\mathrm{pcm}$, solvent $=\mathrm{THF})$

Optimized Structure:

\begin{tabular}{|c|c|c|c|c|c|}
\hline \multirow{3}{*}{$\begin{array}{l}\text { Center } \\
\text { Number } \\
-1\end{array}$} & \multirow{2}{*}{\multicolumn{2}{|c|}{$\begin{array}{l}\text { Atomic } \\
\text { Number }\end{array}$}} & \multirow{2}{*}{$\begin{array}{l}\text { Atomic } \\
\text { Type }\end{array}$} & \multicolumn{2}{|c|}{ Coordinates (Angstroms } \\
\hline & & & & $\mathrm{X}$ & Z \\
\hline & 7 & 0 & -0.233687 & -0.871282 & 2.143634 \\
\hline 2 & 6 & 0 & 0.501345 & -2.053877 & 2.216756 \\
\hline 3 & 6 & 0 & -1.675668 & -1.135921 & 2.193568 \\
\hline 4 & 6 & 0 & 0.223047 & 0.304306 & 3.006645 \\
\hline 5 & 1 & 0 & 1.478833 & -1.926159 & 2.680243 \\
\hline 6 & 3 & 0 & -1.782749 & -0.044735 & 0.299168 \\
\hline 7 & 1 & 0 & -2.058929 & -0.661720 & 3.101463 \\
\hline 8 & 6 & 0 & -0.405650 & -3.169781 & 2.689512 \\
\hline 9 & 6 & 0 & -1.801353 & -2.658876 & 2.280226 \\
\hline 10 & 1 & 0 & -2.090193 & -3.118438 & 1.323296 \\
\hline 11 & 1 & 0 & -2.579231 & -2.943362 & 3.004248 \\
\hline 12 & 1 & 0 & -0.182404 & -4.147991 & 2.237039 \\
\hline 13 & 1 & 0 & -0.348564 & -3.304717 & 3.789047 \\
\hline 14 & 6 & 0 & 1.648512 & 0.728525 & 2.672909 \\
\hline 15 & 6 & 0 & -0.716741 & 1.500022 & 2.846468 \\
\hline 16 & 6 & 0 & 0.196154 & -0.162379 & 4.470631 \\
\hline 17 & 1 & 0 & 1.726178 & 1.178649 & 1.680222 \\
\hline 18 & 1 & 0 & 1.963435 & 1.469481 & 3.421379 \\
\hline 19 & 1 & 0 & 2.337837 & -0.117801 & 2.725286 \\
\hline 20 & 1 & 0 & -0.874051 & 1.735644 & 1.790316 \\
\hline 21 & 1 & 0 & -1.691046 & 1.329276 & 3.315741 \\
\hline 22 & 1 & 0 & -0.256266 & 2.369610 & 3.332381 \\
\hline 23 & 1 & 0 & 0.978513 & -0.905756 & 4.660283 \\
\hline 24 & 1 & 0 & 0.382622 & 0.707141 & 5.113944 \\
\hline 25 & 1 & 0 & -0.773033 & -0.591468 & 4.745206 \\
\hline 26 & 3 & 0 & 1.264658 & -1.515906 & 0.142334 \\
\hline 27 & 8 & 0 & 0.034139 & -0.190401 & 0.481856 \\
\hline 28 & 3 & 0 & 0.449240 & 1.469440 & -0.177949 \\
\hline 29 & 7 & 0 & 1.891278 & 2.610260 & -0.698061 \\
\hline 30 & 6 & 0 & 2.713906 & 2.241023 & -1.826289 \\
\hline 31 & 6 & 0 & 2.404530 & 3.765263 & 0.006683 \\
\hline 32 & 6 & 0 & 2.290648 & 0.872845 & -2.358146 \\
\hline 33 & 6 & 0 & 2.677549 & 3.241819 & -2.998814 \\
\hline 34 & 1 & 0 & 3.790403 & 2.142726 & -1.543589 \\
\hline 35 & 6 & 0 & 1.329599 & 4.333918 & 0.930879 \\
\hline 36 & 6 & 0 & 3.668360 & 3.480416 & 0.847121 \\
\hline 37 & 1 & 0 & 2.680971 & 4.588988 & -0.689363 \\
\hline 38 & 1 & 0 & 2.277212 & 0.114967 & -1.560729 \\
\hline 39 & 1 & 0 & 1.276751 & 0.930864 & -2.784840 \\
\hline
\end{tabular}




\begin{tabular}{|c|c|c|c|c|c|}
\hline 40 & 1 & 0 & 2.967682 & 0.526393 & -3.153028 \\
\hline 41 & 1 & 0 & 1.647379 & 3.315526 & -3.381586 \\
\hline 42 & 1 & 0 & 2.999693 & 4.243988 & -2.689194 \\
\hline 43 & 1 & 0 & 3.334747 & 2.918690 & -3.821451 \\
\hline 44 & 1 & 0 & 0.971572 & 3.544919 & 1.610553 \\
\hline 45 & 1 & 0 & 1.717270 & 5.160154 & 1.544580 \\
\hline 46 & 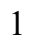 & 0 & 0.477769 & 4.705256 & 0.346584 \\
\hline 47 & & 0 & 4.455887 & 3.005327 & 0.245739 \\
\hline 48 & . & 0 & 4.083483 & 4.408310 & 1.270548 \\
\hline 49 & 1 & 0 & 3.417644 & 2.802662 & 1.676868 \\
\hline 50 & 7 & 0 & -3.952555 & -0.493345 & 0.097903 \\
\hline 51 & 1 & 0 & -3.552138 & -1.036370 & 0.868862 \\
\hline 52 & 6 & 0 & -4.519264 & 0.722648 & 0.711057 \\
\hline 53 & 6 & 0 & -4.913002 & -1.332335 & -0.64568 \\
\hline 54 & 6 & 0 & -5.139918 & 1.630101 & -0.34385 \\
\hline 55 & 6 & 0 & -5.500364 & 0.417254 & 1.84526 \\
\hline 56 & 1 & 0 & -3.663826 & 1.255981 & 1.15895 \\
\hline 57 & 6 & 0 & -4.645227 & -1.253343 & -2.14523 \\
\hline 58 & 6 & 0 & -4.843470 & -2.770464 & -0.14358 \\
\hline 59 & 1 & 0 & -5.936369 & -0.969351 & -0.46285 \\
\hline 60 & 1 & 0 & -4.426342 & 1.826814 & -1.15259 \\
\hline 61 & 1 & 0 & -6.040332 & 1.177756 & -0.78076 \\
\hline 62 & 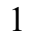 & 0 & -5.436145 & 2.586188 & 0.10676 \\
\hline 63 & 1 & 0 & -6.381897 & -0.126126 & 1.47807 \\
\hline 64 & 1 & 0 & -5.010338 & -0.194311 & 2.614783 \\
\hline 65 & 1 & 0 & -5.849045 & 1.347415 & 2.313742 \\
\hline 66 & 1 & 0 & -4.690710 & -0.215088 & -2.497609 \\
\hline 67 & 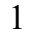 & 0 & -3.643014 & -1.647733 & -2.36298 \\
\hline 68 & & 0 & -5.383319 & -1.842521 & -2.70637 \\
\hline 69 & & 0 & -3.820555 & -3.159320 & -0.24039 \\
\hline 70 & 1 & 0 & -5.131053 & -2.825507 & 0.91525 \\
\hline 71 & 1 & 0 & -5.515093 & -3.418340 & -0.72086 \\
\hline 72 & 7 & 0 & 1.322889 & -2.790131 & -1.742742 \\
\hline 73 & 1 & 0 & 1.531959 & -3.686100 & -1.295600 \\
\hline 74 & 6 & 0 & -0.076309 & -2.855375 & -2.21162 \\
\hline 75 & ( & 0 & 2.311166 & -2.617214 & -2.83111 \\
\hline 76 & ( & 0 & -0.974474 & -3.230679 & -1.04456 \\
\hline 77 & ( & 0 & -0.494146 & -1.507154 & -2.78493 \\
\hline 78 & 1 & 0 & -0.185806 & -3.629035 & -2.98925 \\
\hline 79 & 6 & 0 & 3.713391 & -2.677495 & -2.24400 \\
\hline 80 & 6 & 0 & 2.163012 & -3.628095 & -3.97120 \\
\hline 81 & 1 & 0 & 2.157651 & -1.609794 & -3.236934 \\
\hline 82 & 1 & 0 & -0.635994 & -4.157382 & -0.55838 \\
\hline 83 & 1 & 0 & -0.976429 & -2.425730 & -0.2972 \\
\hline 84 & 1 & 0 & -1.998952 & -3.391454 & -1.40412 \\
\hline 85 & 1 & 0 & -0.349717 & -0.737831 & -2.01222 \\
\hline 86 & 1 & 0 & 0.084260 & -1.231715 & -3.67590 \\
\hline 87 & 1 & 0 & -1.553558 & -1.535162 & -3.06908 \\
\hline 88 & 1 & 0 & 3.844488 & -1.896582 & -1.490032 \\
\hline 89 & 1 & 0 & 3.890372 & -3.657196 & -1.77298 \\
\hline 90 & 1 & 0 & 4.461520 & -2.543525 & -3.03507 \\
\hline 91 & 1 & 0 & 1.232941 & -3.492149 & -4.53521 \\
\hline 92 & 1 & 0 & 2.997089 & -3.514040 & -4.67540 \\
\hline
\end{tabular}




$\begin{array}{rrrrrr}93 & 1 & 0 & 2.185291 & -4.656356 & -3.579289 \\ 94 & 8 & 0 & -1.671285 & 1.924319 & -0.617115 \\ 95 & 6 & 0 & -2.043564 & 3.261725 & -0.223677 \\ 96 & 6 & 0 & -1.866700 & 4.103828 & -1.477102 \\ 97 & 1 & 0 & -1.393284 & 3.559101 & 0.605272 \\ 98 & 1 & 0 & -3.087720 & 3.260928 & 0.118290 \\ 99 & 6 & 0 & -2.319757 & 3.132156 & -2.570586 \\ 100 & 1 & 0 & -0.807342 & 4.358881 & -1.608092 \\ 101 & 1 & 0 & -2.458860 & 5.024359 & -1.446163 \\ 102 & 6 & 0 & -1.803627 & 1.789365 & -2.054161 \\ 103 & 1 & 0 & -3.413855 & 3.127835 & -2.650984 \\ 104 & 1 & 0 & -1.904179 & 3.370862 & -3.554891 \\ 105 & 1 & 0 & -2.478893 & 0.947961 & -2.258037 \\ 106 & 1 & 0 & -0.809929 & 1.561048 & -2.462352 \\ 107 & 8 & 0 & 3.189910 & -1.200099 & 0.586191 \\ 108 & 6 & 0 & 3.978771 & -2.191364 & 1.239552 \\ 109 & 6 & 0 & 4.725150 & -1.426882 & 2.334361 \\ 110 & 1 & 0 & 4.684673 & -2.633427 & 0.516587 \\ 111 & 1 & 0 & 3.300408 & -2.970128 & 1.605861 \\ 112 & 6 & 0 & 4.892056 & -0.013767 & 1.728127 \\ 113 & 1 & 0 & 5.682420 & -1.898715 & 2.579668 \\ 114 & 1 & 0 & 4.120935 & -1.391190 & 3.249093 \\ 115 & 6 & 0 & 4.023844 & -0.042301 & 0.457720 \\ 116 & 1 & 0 & 4.547286 & 0.760041 & 2.423222 \\ 117 & 1 & 0 & 5.936802 & 0.201559 & 1.479738 \\ 118 & 1 & 0 & 3.370123 & 0.828033 & 0.327686 \\ 119 & 1 & 0 & 4.652445 & -0.156667 & -0.442019 \\ & & & & & \end{array}$

\section{Thermodynamics:}

Zero-point correction $=$ Thermal correction to Energy= Thermal correction to Enthalpy= Thermal correction to Gibbs Free Energy= Sum of electronic and zero-point Energies= Sum of electronic and thermal Energies= Sum of electronic and thermal Enthalpies= Sum of electronic and thermal Free Energies=
1.073089 (Hartree/Particle)

1.130793

1.131737

0.984124

$-1806.363685$

$-1806.305981$

$-1806.305036$

$-1806.452650$ 


\section{Energy Decomposition Analysis Calculations}

\section{Structure $22-\mathrm{Li}_{2} \mathrm{O}$ portion frequency sweep only}

Input:

\#m062x/jul-cc-pVDZ freq Pop=Hirshfeld scrf=(pcm,solvent=THF $)$

Input Structure:

Symbolic Z-matrix:

Charge $=-1$ Multiplicity $=1$

$\begin{array}{lccc}\mathrm{O} & 0.09746 & -0.03464 & -0.53427 \\ \mathrm{Li} & -0.64371 & 1.66835 & -0.293 \\ \mathrm{O} & 0.60541 & 3.27143 & 0.21876 \\ \mathrm{C} & 0.37848 & 4.27008 & -0.79554 \\ \mathrm{C} & 1.26477 & 5.44486 & -0.40397 \\ \mathrm{C} & 2.47555 & 4.72043 & 0.18816 \\ \mathrm{C} & 1.8138 & 3.56462 & 0.93537 \\ \mathrm{H} & 0.65265 & 3.84675 & -1.77111 \\ \mathrm{H} & -0.69537 & 4.49626 & -0.8101 \\ \mathrm{H} & 1.51342 & 6.0814 & -1.25965 \\ \mathrm{H} & 0.77337 & 6.06153 & 0.36046 \\ \mathrm{H} & 3.11368 & 4.33183 & -0.61686 \\ \mathrm{H} & 3.08652 & 5.34802 & 0.84577 \\ \mathrm{H} & 2.44144 & 2.66506 & 0.96381 \\ \mathrm{H} & 1.54866 & 3.84271 & 1.96736 \\ \mathrm{~N} & -1.84294 & 2.21622 & 1.29748 \\ \mathrm{H} & -1.55393 & 3.19278 & 1.30966 \\ \mathrm{C} & -1.15357 & 1.57119 & 2.4367 \\ \mathrm{C} & -3.31972 & 2.16514 & 1.29232 \\ \mathrm{H} & -0.08353 & 1.61667 & 2.17857 \\ \mathrm{C} & -1.34722 & 2.32625 & 3.75124 \\ \mathrm{C} & -1.53741 & 0.10311 & 2.56631 \\ \mathrm{H} & -3.69412 & 1.97528 & 2.31201 \\ \mathrm{C} & -3.85087 & 3.51844 & 0.83267 \\ \mathrm{C} & -3.81219 & 1.06011 & 0.3639 \\ \mathrm{H} & -1.03502 & 3.37143 & 3.64708 \\ \mathrm{H} & -2.39369 & 2.30904 & 4.07473 \\ \mathrm{H} & -0.74709 & 1.86887 & 4.54777 \\ \mathrm{H} & -1.36524 & -0.43171 & 1.62222 \\ \mathrm{H} & -0.9322 & -0.37589 & 3.34637 \\ \mathrm{H} & -2.59911 & -0.0036 & 2.84357 \\ \mathrm{H} & -3.55736 & 4.31757 & 1.5288 \\ \mathrm{H} & -3.44935 & 3.75383 & -0.16469 \\ \mathrm{H} & -4.94546 & 3.50693 & 0.76484 \\ \mathrm{H} & -3.48387 & 1.2788 & -0.66113 \\ \mathrm{H} & -3.41498 & 0.07876 & 0.647 \\ \mathrm{H} & -4.90871 & 1.00621 & 0.3778\end{array}$




$\begin{array}{lccc}\mathrm{N} & -0.27547 & -3.37559 & 0.01738 \\ \mathrm{C} & 0.09777 & -4.73412 & -0.29425 \\ \mathrm{C} & -1.68487 & -3.17719 & 0.30705 \\ \mathrm{H} & -0.62903 & -5.46646 & 0.11568 \\ \mathrm{C} & 0.18889 & -5.004 & -1.81114 \\ \mathrm{C} & 1.45122 & -5.07183 & 0.33963 \\ \mathrm{H} & -1.85811 & -2.07784 & 0.29601 \\ \mathrm{C} & -2.67933 & -3.74501 & -0.72377 \\ \mathrm{C} & -2.08405 & -3.65306 & 1.71742 \\ \mathrm{H} & 0.45397 & -6.04984 & -2.03254 \\ \mathrm{H} & -0.75916 & -4.77537 & -2.31043 \\ \mathrm{H} & 0.9639 & -4.35572 & -2.24881 \\ \mathrm{H} & 2.18326 & -4.29784 & 0.05594 \\ \mathrm{H} & 1.36766 & -5.08579 & 1.43383 \\ \mathrm{H} & 1.83794 & -6.04606 & 0.00688 \\ \mathrm{H} & -2.62601 & -4.84302 & -0.75478 \\ \mathrm{H} & -3.71265 & -3.46803 & -0.46653 \\ \mathrm{H} & -2.4603 & -3.36525 & -1.73024 \\ \mathrm{H} & -1.90381 & -4.73345 & 1.81511 \\ \mathrm{H} & -1.46789 & -3.13917 & 2.46722 \\ \mathrm{H} & -3.14595 & -3.46359 & 1.94028 \\ \mathrm{Li} & 0.37392 & -1.67423 & 0.32185 \\ \mathrm{O} & 1.36764 & -1.14393 & 1.9106 \\ \mathrm{C} & 2.16035 & 0.03797 & 1.88726 \\ \mathrm{C} & 3.63125 & -0.42703 & 1.8014 \\ \mathrm{C} & 3.54055 & -1.96217 & 1.937 \\ \mathrm{C} & 2.1495 & -2.16977 & 2.52739 \\ \mathrm{H} & 1.81319 & 0.61469 & 1.02409 \\ \mathrm{H} & 1.97898 & 0.60885 & 2.81041 \\ \mathrm{H} & 4.09793 & -0.12936 & 0.85417 \\ \mathrm{H} & 4.22287 & 0.00558 & 2.61593 \\ \mathrm{H} & 3.58576 & -2.45118 & 0.95126 \\ \mathrm{H} & 4.33219 & -2.39107 & 2.56897 \\ \mathrm{H} & 1.66967 & -3.12338 & 2.27568 \\ \mathrm{H} & 2.14605 & -2.02348 & 3.62028\end{array}$

Thermodynamics:

Zero-point correction $=$

Thermal correction to Energy=

Thermal correction to Enthalpy=

Thermal correction to Gibbs Free Energy=

Sum of electronic and zero-point Energies=

Sum of electronic and thermal Energies=

Sum of electronic and thermal Enthalpies=

Sum of electronic and thermal Free Energies=
0.640461 (Hartree/Particle)

0.671414

0.672358

0.578130

$-1138.537293$

$-1138.506341$

$-1138.505396$

$-1138.599625$ 


\section{Structure 22 - Pyrrolidine portion frequency sweep only}

Input:

\#m062x/jul-cc-pVDZ freq Pop=Hirshfeld scrf $=(\mathrm{pcm}$,solvent=THF $)$

Input Structure:

Symbolic Z-matrix:

Charge $=1$ Multiplicity $=1$

$\begin{array}{llll}\mathrm{N} & 0.10857 & 0.03935 & -1.94184 \\ \mathrm{C} & 1.5781 & -0.0122 & -2.38216 \\ \mathrm{C} & -0.6895 & -1.1286 & -2.46579 \\ \mathrm{C} & -0.64758 & 1.32208 & -2.36052 \\ \mathrm{C} & -2.11076 & -0.6024 & -2.49023 \\ \mathrm{C} & -1.91418 & 0.80377 & -3.06038 \\ \mathrm{H} & -0.51478 & -2.00521 & -1.82751 \\ \mathrm{H} & -0.36631 & -1.32782 & -3.49274 \\ \mathrm{H} & -2.51152 & -0.57339 & -1.47069 \\ \mathrm{H} & -2.76096 & -1.23844 & -3.10177 \\ \mathrm{H} & -2.78489 & 1.44679 & -2.86728 \\ \mathrm{H} & -1.81635 & 0.72413 & -4.16032 \\ \mathrm{H} & -0.00326 & 1.83733 & -3.08351 \\ \mathrm{C} & 2.25432 & -1.20755 & -1.70547 \\ \mathrm{C} & 1.69459 & -0.15235 & -3.90329 \\ \mathrm{C} & 2.27096 & 1.27661 & -1.93271 \\ \mathrm{H} & 1.66403 & -2.12818 & -1.79825 \\ \mathrm{H} & 3.22935 & -1.37339 & -2.18261 \\ \mathrm{H} & 2.42871 & -1.00579 & -0.64237 \\ \mathrm{H} & 1.5518 & -1.18798 & -4.23135 \\ \mathrm{H} & 0.97892 & 0.49198 & -4.42683 \\ \mathrm{H} & 2.707 & 0.14969 & -4.19986 \\ \mathrm{H} & 2.01796 & 2.12142 & -2.58269 \\ \mathrm{H} & 1.99042 & 1.5239 & -0.905 \\ \mathrm{H} & 3.35658 & 1.1221 & -1.98406\end{array}$

Thermodynamics:

Zero-point correction $=$

Thermal correction to Energy=

Thermal correction to Enthalpy=

Thermal correction to Gibbs Free Energy=

Sum of electronic and zero-point Energies=

Sum of electronic and thermal Energies=

Sum of electronic and thermal Enthalpies=

Sum of electronic and thermal Free Energies=
0.227183 (Hartree/Particle)

0.237112

0.238056

0.193556

$-368.576961$

$-368.567032$

$-368.566088$

$-368.610588$ 


\section{Structure $22_{\mathrm{TS}-\mathrm{A}}-\mathrm{Li}_{2} \mathrm{O}$ portion frequency sweep only}

Input:

\#m062x/jul-cc-pVDZ freq Pop=Hirshfeld scrf=(pcm,solvent=THF $)$

Input Structure:

Symbolic Z-matrix:

Charge $=-1$ Multiplicity $=1$

$\mathrm{O} 2$

$\begin{array}{lll}0.12639 & -0.20446 & 0.3991\end{array}$

$\begin{array}{llll}\text { LI27 } & -1.50869 & 0.47349 & 0.37032\end{array}$

$\begin{array}{llll}\mathrm{O} 28 & -3.18476 & -0.60086 & 0.15001\end{array}$

$\begin{array}{llll}\mathrm{C} 29 & -4.24481 & -0.43285 & 1.10785\end{array}$

$\begin{array}{llll}\text { C30 } & -5.48657 & -1.00487 & 0.43803\end{array}$

$\begin{array}{llll}\text { C31 } & -4.88441 & -2.15631 & -0.37062\end{array}$

$\begin{array}{lllll}\mathrm{C} 32 & -3.58235 & -1.53837 & -0.86814\end{array}$

$\begin{array}{llll}\mathrm{H} 33 & -3.99519 & -0.99484 & 2.0203\end{array}$

$\begin{array}{llll}\mathrm{H} 34 & -4.31714 & 0.63452 & 1.35365\end{array}$

$\begin{array}{llll}\mathrm{H} 35 & -6.23891 & -1.32847 & 1.16427\end{array}$

H36 $\quad-5.93755 \quad-0.26031 \quad-0.23114$

$\begin{array}{llll}\mathrm{H} 37 & -4.6732 & -3.01119 & 0.285\end{array}$

H38 $\quad-5.52272 \quad-2.49367-1.19337$

H39 $\quad-2.77563 \quad-2.26892-1.00518$

$\begin{array}{lllll}\mathrm{H} 40 & -3.73321 & -0.98869 & -1.80929\end{array}$

$\begin{array}{lllll}\mathrm{N} 41 & -2.22731 & 2.01608 & -0.82439\end{array}$

$\begin{array}{lllll}\mathrm{H} 42 & -3.22701 & 1.82524 & -0.76653\end{array}$

$\begin{array}{lllll}\mathrm{C} 43 & -1.77387 & 1.43486 & -2.11179\end{array}$

$\begin{array}{lllll}\text { C44 } & -2.02924 & 3.47279 & -0.66196\end{array}$

$\begin{array}{llll}\mathrm{H} 45 & -1.88493 & 0.34632 & -1.98764\end{array}$

$\begin{array}{lllll}\mathrm{C} 46 & -2.64985 & 1.85736 & -3.29103\end{array}$

$\begin{array}{lllll}\mathrm{C} 47 & -0.29752 & 1.70874 & -2.36454\end{array}$

H48 $\quad-1.89753 \quad 3.94349-1.64973$

$\begin{array}{lllll}\text { C49 } & -3.27254 & 4.07149 & -0.01446\end{array}$

$\begin{array}{lllll}\text { C50 } & -0.79229 & 3.75961 & 0.18425\end{array}$

$\begin{array}{llll}\text { H51 } & -3.70267 & 1.60247 & -3.10584\end{array}$

$\begin{array}{llll}\mathrm{H} 52 & -2.58112 & 2.93855 & -3.47328\end{array}$

$\begin{array}{lllll}\mathrm{H} 53 & -2.32893 & 1.34149 & -4.20572\end{array}$

$\begin{array}{llll}\mathrm{H} 54 & 0.29852 & 1.38973 & -1.50013\end{array}$

$\begin{array}{lllll}\text { H55 } & 0.03368 & 1.13997 & -3.24287\end{array}$

H56 $\quad-0.11388 \quad 2.77426 \quad-2.56187$

$\begin{array}{lllll}\mathrm{H} 57 & -4.15298 & 3.94774 & -0.65979\end{array}$

$\begin{array}{lllll}\mathrm{H} 58 & -3.4701 & 3.57464 & 0.94761\end{array}$

$\begin{array}{llll}\text { H59 } & -3.13241 & 5.14207 & 0.17777\end{array}$

$\begin{array}{llll}\mathrm{H} 60 & -0.95379 & 3.39182 & 1.20804\end{array}$

$\begin{array}{llll}\text { H61 } & 0.10108 & 3.2688 & -0.21848\end{array}$

$\begin{array}{lllll}\text { H62 } & -0.60589 & 4.84114 & 0.23187\end{array}$

$\begin{array}{lllll}\text { N64 } & 3.31506 & 0.62174 & -0.24781\end{array}$

$\begin{array}{lllll}\text { C65 } & 4.74017 & 0.46543 & -0.39532\end{array}$

$\begin{array}{llll}\text { C66 } & 2.87069 & 1.98338 & -0.01854\end{array}$

$\begin{array}{lllll}\mathrm{H} 67 & 5.22823 & 1.40448 & -0.73853\end{array}$ 


\begin{tabular}{lccc} 
C68 & 5.45973 & 0.05739 & 0.91015 \\
C69 & 5.04724 & -0.5981 & -1.45527 \\
H70 & 1.78783 & 1.90618 & 0.19105 \\
C71 & 3.48682 & 2.70952 & 1.19482 \\
C72 & 3.00533 & 2.89746 & -1.25622 \\
H73 & 6.54961 & -0.0398 & 0.77081 \\
H74 & 5.27622 & 0.78849 & 1.70632 \\
H75 & 5.06464 & -0.91405 & 1.24781 \\
H76 & 4.55447 & -1.54088 & -1.16454 \\
H77 & 4.64733 & -0.29099 & -2.43165 \\
H78 & 6.12636 & -0.79271 & -1.55558 \\
H79 & 4.56239 & 2.88662 & 1.0412 \\
H80 & 3.00617 & 3.68825 & 1.35381 \\
H81 & 3.36531 & 2.11234 & 2.10887 \\
H82 & 4.06299 & 3.04312 & -1.52308 \\
H83 & 2.49801 & 2.43591 & -2.11388 \\
H84 & 2.56668 & 3.89388 & -1.0806 \\
LI85 & 1.69476 & -0.34688 & -0.52737 \\
O86 & 1.23706 & -1.40974 & -2.19586 \\
C87 & -0.11669 & -1.87515 & -2.21633 \\
C88 & -0.03261 & -3.41504 & -2.20898 \\
C89 & 1.45222 & -3.70938 & -2.52281 \\
C90 & 1.98649 & -2.34722 & -2.9647 \\
H91 & -0.58979 & -1.43517 & -1.32991 \\
H92 & -0.60224 & -1.51148 & -3.13796 \\
H93 & -0.31907 & -3.82468 & -1.23305 \\
H94 & -0.7001 & -3.84656 & -2.9632 \\
H95 & 1.98077 & -4.03974 & -1.62032 \\
H96 & 1.5805 & -4.47513 & -3.29533 \\
H97 & 3.04768 & -2.18386 & -2.75776 \\
H98 & 1.79327 & -2.17477 & -4.0367 \\
& & & \\
\hline & & &
\end{tabular}

\section{Thermodynamics:}

Zero-point correction $=$ Thermal correction to Energy= Thermal correction to Enthalpy= Thermal correction to Gibbs Free Energy= Sum of electronic and zero-point Energies= Sum of electronic and thermal Energies= Sum of electronic and thermal Enthalpies= Sum of electronic and thermal Free Energies=
0.640503 (Hartree/Particle) 0.672158 0.673103 0.575531 $-1138.547845$ $-1138.516190$ $-1138.515246$

$-1138.612818$ 


\section{Structure 22 2 TS-A - Pyrrolidine portion frequency sweep only}

Input:

\#m062x/jul-cc-pVDZ freq Pop=Hirshfeld scrf $=(\mathrm{pcm}$, solvent=THF $)$

Input Structure:

Symbolic Z-matrix:

Charge $=1$ Multiplicity $=1$

$\begin{array}{lccl}\text { N1 } & 0.07871 & -0.69653 & 2.22697 \\ \text { C3 } & 0.23724 & -2.19639 & 2.11364 \\ \text { C4 } & 1.27037 & 0.10005 & 2.63663 \\ \text { C5 } & -0.98402 & -0.2123 & 2.87051 \\ \text { C6 } & 0.68155 & 1.47125 & 2.95446 \\ \text { C7 } & -0.76257 & 1.17396 & 3.40524 \\ \text { H8 } & 2.00766 & 0.11941 & 1.8313 \\ \text { H9 } & 1.68189 & -0.37022 & 3.54097 \\ \text { H10 } & 0.68488 & 2.0764 & 2.04301 \\ \text { H11 } & 1.26757 & 1.99041 & 3.72052 \\ \text { H12 } & -1.47054 & 1.92191 & 3.01045 \\ \text { H13 } & -0.86268 & 1.20596 & 4.50226 \\ \text { H14 } & -1.92534 & -0.747 & 2.8557 \\ \text { C15 } & 1.41957 & -2.56386 & 1.21872 \\ \text { C16 } & 0.47469 & -2.77071 & 3.51793 \\ \text { C17 } & -1.04151 & -2.78446 & 1.52368 \\ \text { H18 } & 2.32129 & -1.99155 & 1.46671 \\ \text { H19 } & 1.64407 & -3.62802 & 1.37435 \\ \text { H20 } & 1.16847 & -2.40861 & 0.16653 \\ \text { H21 } & 1.44974 & -2.47217 & 3.92141 \\ \text { H22 } & -0.3135 & -2.45629 & 4.21006 \\ \text { H23 } & 0.46517 & -3.86566 & 3.44816 \\ \text { H24 } & -1.88217 & -2.74383 & 2.22754 \\ \text { H25 } & -1.29338 & -2.23473 & 0.61233 \\ \text { H26 } & -0.85935 & -3.83989 & 1.28378\end{array}$

Thermodynamics:

Zero-point correction $=$

Thermal correction to Energy=

Thermal correction to Enthalpy=

Thermal correction to Gibbs Free Energy=

Sum of electronic and zero-point Energies=

Sum of electronic and thermal Energies=

Sum of electronic and thermal Enthalpies=

Sum of electronic and thermal Free Energies=
0.232149 (Hartree/Particle)

0.241802

0.242746

0.198147

$-368.681698$

$-368.672045$

$-368.671101$

$-368.715700$ 


\section{Structure $23-\mathrm{Li}_{2} \mathrm{O}$ portion frequency sweep only}

Input:

\#m062x/jul-cc-pVDZ freq Pop=Hirshfeld scrf=(pcm,solvent=THF)

Input Structure:

Symbolic Z-matrix:

Charge $=0$ Multiplicity $=1$

$\mathrm{O} 2$

$\begin{array}{lll}-0.01262 & -0.80406 & 0.27556\end{array}$

$\begin{array}{lllll}\mathrm{H} 8 & 3.14666 & 0.63018 & 1.33093\end{array}$

$\begin{array}{llll}\text { LI27 } & -1.46617 & 0.3508 & 0.52103\end{array}$

$\begin{array}{llll}\mathrm{O} 28 & -3.28617 & -0.21174 & -0.31749\end{array}$

$\begin{array}{llll}\mathrm{C} 29 & -4.36902 & -0.36582 & 0.61941\end{array}$

$\begin{array}{llll}\text { C30 } & -5.45279 & -1.1566 & -0.11412\end{array}$

C31 $\quad-4.64301-1.93949-1.15205$

C32 $\quad-3.57599-0.92047-1.52842$

$\begin{array}{llll}\mathrm{H} 33 & -3.9801 & -0.894 & 1.49877\end{array}$

$\begin{array}{llll}\mathrm{H} 34 & -4.70669 & 0.63159 & 0.93253\end{array}$

H35 $\quad-6.02336 \quad-1.79826 \quad 0.56507$

H36 $\quad-6.15111-0.47519-0.61667$

H37 $\quad-4.178 \quad-2.82178-0.69437$

H38 $\quad-5.23875 \quad-2.25821 \quad-2.01378$

H39 $\quad-2.64056-1.36196-1.89322$

$\mathrm{H} 40 \quad-3.95354 \quad-0.21126-2.28267$

N41 $\quad-2.00833 \quad 2.29367 \quad-0.15797$

$\begin{array}{lllll}\mathrm{H} 42 & -2.96983 & 1.99814 & -0.00079\end{array}$

$\begin{array}{lllll}\mathrm{C} 43 & -1.85826 & 2.36583 & -1.62572\end{array}$

$\begin{array}{lllll}\text { C44 } & -1.79168 & 3.53946 & 0.6062\end{array}$

$\begin{array}{lllll}\mathrm{H} 45 & -1.9621 & 1.32466 & -1.97123\end{array}$

$\begin{array}{llll}\text { C46 } & -2.9596 & 3.1868 & -2.2982\end{array}$

$\begin{array}{lllll}\mathrm{C} 47 & -0.47084 & 2.84558 & -2.02618\end{array}$

$\begin{array}{lllll}\mathrm{H} 48 & -1.92035 & 4.41674 & -0.05034\end{array}$

$\begin{array}{llll}\text { C49 } & -2.84111 & 3.6116 & 1.7109\end{array}$

$\begin{array}{llll}\text { C50 } & -0.38775 & 3.57365 & 1.20229\end{array}$

$\begin{array}{lllll}\text { H51 } & -3.94998 & 2.79067 & -2.03439\end{array}$

H52 $\quad-2.91507 \quad 4.24181-1.99509$

$\begin{array}{llll}\text { H53 } & -2.85509 & 3.14587 & -3.39057\end{array}$

H54 $\quad 0.30064 \quad 2.23984-1.53786$

$\begin{array}{lllll}\text { H55 } & -0.34659 & 2.75807 & -3.11326\end{array}$

H56 $\quad-0.31576 \quad 3.89912-1.7541$

$\begin{array}{llll}\mathrm{H} 57 & -3.85327 & 3.67581 & 1.28756\end{array}$

$\begin{array}{llll}\mathrm{H} 58 & -2.78105 & 2.70982 & 2.33882\end{array}$

$\begin{array}{llll}\text { H59 } & -2.67746 & 4.48908 & 2.34805\end{array}$

$\begin{array}{llll}\text { H60 } & -0.23924 & 2.69094 & 1.84104\end{array}$

$\begin{array}{llll}\text { H61 } & 0.38145 & 3.57102 & 0.42291\end{array}$

$\begin{array}{llll}\mathrm{H} 62 & -0.2533 & 4.47956 & 1.80908\end{array}$

$\begin{array}{lllll}\mathrm{N} 64 & 3.34353 & 0.69604 & 0.33327\end{array}$

$\begin{array}{lllll}\text { C65 } & 4.79049 & 0.4924 & 0.12392\end{array}$

$\begin{array}{llll}\text { C66 } & 2.78458 & 1.97214 & -0.14549\end{array}$ 


$\begin{array}{llll}\text { H67 } & 5.26452 & 1.44944 & -0.1487 \\ \text { C68 } & 5.43296 & 0.00797 & 1.41971 \\ \text { C69 } & 5.03132 & -0.50355 & -1.00823 \\ \text { H70 } & 1.69961 & 1.87805 & 0.02156 \\ \text { C71 } & 3.27345 & 3.17983 & 0.65598 \\ \text { C72 } & 3.01888 & 2.15059 & -1.64128 \\ \text { H73 } & 6.50285 & -0.18718 & 1.27354 \\ \text { H74 } & 5.32177 & 0.75796 & 2.21477 \\ \text { H75 } & 4.95436 & -0.92414 & 1.75289 \\ \text { H76 } & 4.62924 & -1.48744 & -0.72506 \\ \text { H77 } & 4.52584 & -0.18828 & -1.92821 \\ \text { H78 } & 6.10655 & -0.60839 & -1.20874 \\ \text { H79 } & 4.36183 & 3.30272 & 0.56754 \\ \text { H80 } & 2.80054 & 4.10291 & 0.29349 \\ \text { H81 } & 3.0215 & 3.06044 & 1.71909 \\ \text { H82 } & 4.0877 & 2.26945 & -1.86525 \\ \text { H83 } & 2.64105 & 1.28351 & -2.19754 \\ \text { H84 } & 2.50298 & 3.05265 & -1.99449 \\ \text { LI85 } & 1.8842 & -0.82422 & 0.11383 \\ \text { O86 } & 1.96872 & -1.21088 & -1.86908 \\ \text { C87 } & 0.81043 & -0.74533 & -2.56538 \\ \text { C88 } & 0.02997 & -2.01044 & -2.89086 \\ \text { C89 } & 1.14644 & -3.02862 & -3.18351 \\ \text { C90 } & 2.38131 & -2.44727 & -2.46247 \\ \text { H91 } & 0.2789 & -0.06247 & -1.89326 \\ \text { H92 } & 1.12032 & -0.21373 & -3.48158 \\ \text { H93 } & -0.54545 & -2.30297 & -2.00491 \\ \text { H94 } & -0.65411 & -1.87743 & -3.73652 \\ \text { H95 } & 0.89553 & -4.0288 & -2.81389 \\ \text { H96 } & 1.33317 & -3.10527 & -4.26049 \\ \text { H97 } & 2.74944 & -3.09134 & -1.6548 \\ \text { H98 } & 3.20701 & -2.25534 & -3.16303\end{array}$

Thermodynamics:

Zero-point correction=

Thermal correction to Energy=

Thermal correction to Enthalpy=

Thermal correction to Gibbs Free Energy=

Sum of electronic and zero-point Energies=

Sum of electronic and thermal Energies=

Sum of electronic and thermal Enthalpies=

Sum of electronic and thermal Free Energies=
0.656987 (Hartree/Particle)

0.690400

0.691345

0.590100

$-1139.073309$

$-1139.039896$

$-1139.038952$

$-1139.1401968$ 


\section{Structure 23 - Pyrrolidine portion frequency sweep only}

Input:

\#m062x/jul-cc-pVDZ freq Pop=Hirshfeld scrf=(pcm,solvent=THF)

Input Structure:

Symbolic Z-matrix:

Charge $=0$ Multiplicity $=1$

N1 $\quad-0.0496-1.33041 \quad 1.6024$

$\begin{array}{llll}\mathrm{C} 3 & -0.43623 & -2.81022 & 1.48649\end{array}$

$\begin{array}{llll}\mathrm{C} 4 & 1.35091 & -1.14724 & 2.17935\end{array}$

$\begin{array}{llll}\text { C5 } & -1.06851 & -0.47816 & 2.40922\end{array}$

$\begin{array}{llll}\text { C6 } & 1.19675 & 0.20549 & 2.87216\end{array}$

$\begin{array}{llll}\text { C7 } & -0.20678 & 0.18136 & 3.49302\end{array}$

H9 $\quad \begin{array}{llll}\text { H9 } & 1.42385 & -1.88744 & 2.99177\end{array}$

$\begin{array}{llll}\mathrm{H} 10 & 1.25065 & 1.04288 & 2.15076\end{array}$

$\begin{array}{llll}\mathrm{H} 11 & 1.99691 & 0.35991 & 3.61319\end{array}$

$\begin{array}{llll}\mathrm{H} 12 & -0.56945 & 1.19034 & 3.75384\end{array}$

H13 $\quad-0.15368-0.39724 \quad 4.4383$

$\begin{array}{llll}\text { H14 } & -1.76972 & -1.18734 & 2.86828\end{array}$

$\begin{array}{llll}\mathrm{C} 15 & 0.57699 & -3.53663 & 0.59734\end{array}$

$\begin{array}{llll}\mathrm{C} 16 & -0.46037 & -3.49261 & 2.85927\end{array}$

$\begin{array}{lllll}\mathrm{C} 17 & -1.81584 & -2.91593 & 0.83677\end{array}$

$\begin{array}{llll}\mathrm{H} 18 & 1.58167 & -3.49152 & 1.02915\end{array}$

$\begin{array}{llll}\mathrm{H} 19 & 0.27543 & -4.59014 & 0.5143\end{array}$

$\begin{array}{lllll}\mathrm{H} 20 & 0.59633 & -3.09389 & -0.40307\end{array}$

$\begin{array}{lllll}\mathrm{H} 21 & 0.54851 & -3.71973 & 3.21888\end{array}$

$\begin{array}{llll}\mathrm{H} 22 & -0.9739 & -2.88492 & 3.61156\end{array}$

$\begin{array}{llll}\mathrm{H} 23 & -1.0012 & -4.4434 & 2.75993\end{array}$

$\begin{array}{llll}\mathrm{H} 24 & -2.60919 & -2.5622 & 1.50441\end{array}$

$\mathrm{H} 25 \quad-1.83782-2.3292 \quad-0.0851$

$\begin{array}{llll}\mathrm{H} 26 & -2.01412 & -3.97008 & 0.59979\end{array}$

Thermodynamics:

Zero-point correction $=$

Thermal correction to Energy=

0.211653 (Hartree/Particle)

Thermal correction to Enthalpy=

0.221250

0.222194

Thermal correction to Gibbs Free Energy $=\quad 0.178424$

Sum of electronic and zero-point Energies=

$-368.086640$

Sum of electronic and thermal Energies $=$ $-368.077043$

Sum of electronic and thermal Enthalpies= $-368.076099$

Sum of electronic and thermal Free Energies $=\quad-368.119869$ 


\section{Structure $23_{\mathrm{Ts}}-\mathrm{Li}_{2} \mathrm{O}$ portion frequency sweep only}

Input:

\#m062x/jul-cc-pVDZ freq Pop=Hirshfeld scrf=(pcm,solvent=THF)

Input Structure:

Symbolic Z-matrix:

Charge $=0$ Multiplicity $=1$

$\mathrm{O} 2$

$\begin{array}{lll}-0.02572 & -0.73129 & -0.23989\end{array}$

$\begin{array}{lllll}\mathrm{H} 8 & -2.94795 & 0.57051 & -1.34659\end{array}$

$\begin{array}{lllll}\text { LI27 } & 1.39117 & 0.33227 & -0.55411\end{array}$

$\begin{array}{llll}\mathrm{O} 28 & 3.25293 & -0.25809 & 0.24534\end{array}$

$\begin{array}{llll}\mathrm{C} 29 & 4.34898 & -0.42797 & -0.67052\end{array}$

$\begin{array}{llll}\mathrm{C} 30 & 5.36274 & -1.31467 & 0.05055\end{array}$

$\begin{array}{llll}\text { C31 } & 4.46567 & -2.13829 & 0.97937\end{array}$

$\begin{array}{llll}\mathrm{C} 32 & 3.42568 & -1.10331 & 1.39\end{array}$

H33 $\quad 3.95966-0.89487-1.58463$

H34 $\quad 4.74655 \quad 0.56371-0.9246$

H35 $\quad 5.94501-1.92624-0.64641$

$\begin{array}{llll}\mathrm{H} 36 & 6.05703 & -0.702 & 0.64025\end{array}$

$\begin{array}{llll}\mathrm{H} 37 & 3.98698 & -2.95598 & 0.42613\end{array}$

$\begin{array}{llll}\mathrm{H} 38 & 5.00024 & -2.55591 & 1.83906\end{array}$

$\begin{array}{llll}\mathrm{H} 39 & 2.44596 & -1.52703 & 1.64559\end{array}$

$\begin{array}{llll}\mathrm{H} 40 & 3.78466 & -0.49423 & 2.23557\end{array}$

$\begin{array}{llll}\mathrm{N} 41 & 1.94472 & 2.24758 & 0.28452\end{array}$

$\begin{array}{llll}\mathrm{H} 42 & 2.88856 & 1.94348 & 0.05341\end{array}$

$\begin{array}{llll}\mathrm{C} 43 & 1.84599 & 2.12071 & 1.75284\end{array}$

$\begin{array}{lllll}\mathrm{C} 44 & 1.75686 & 3.59402 & -0.28977\end{array}$

$\begin{array}{llll}\mathrm{H} 45 & 1.91592 & 1.03984 & 1.94704\end{array}$

$\begin{array}{llll}\text { C46 } & 3.00611 & 2.79474 & 2.48776\end{array}$

$\begin{array}{llll}\mathrm{C} 47 & 0.49615 & 2.588 & 2.27797\end{array}$

$\begin{array}{llll}\mathrm{H} 48 & 1.97276 & 4.36725 & 0.46778\end{array}$

$\begin{array}{lllll}\text { C49 } & 2.74434 & 3.76448 & -1.43971\end{array}$

$\begin{array}{lllll}\text { C50 } & 0.32767 & 3.78686 & -0.78838\end{array}$

$\begin{array}{llll}\mathrm{H} 51 & 3.96692 & 2.40261 & 2.12598\end{array}$

$\begin{array}{llll}\text { H52 } & 2.99769 & 3.88359 & 2.34196\end{array}$

$\begin{array}{llll}\mathrm{H} 53 & 2.94174 & 2.59948 & 3.56664\end{array}$

$\begin{array}{llll}\mathrm{H} 54 & -0.31963 & 2.06805 & 1.76134\end{array}$

$\begin{array}{llll}\mathrm{H} 55 & 0.42283 & 2.36689 & 3.35066\end{array}$

$\begin{array}{llll}\text { H56 } & 0.36656 & 3.67174 & 2.1503\end{array}$

$\begin{array}{llll}\mathrm{H} 57 & 3.781 \quad 3.71049 & -1.07917\end{array}$

$\begin{array}{lllll}\mathrm{H} 58 & 2.58968 & 2.96591 & -2.18038\end{array}$

$\begin{array}{llll}\mathrm{H} 59 & 2.60172 & 4.7315 & -1.93719\end{array}$

$\begin{array}{lllll}\mathrm{H} 60 & 0.08821 & 3.00932 & -1.52901\end{array}$

$\begin{array}{llll}\text { H61 } & -0.39846 & 3.72038 & 0.02879\end{array}$

$\begin{array}{llll}\mathrm{H} 62 & 0.21924 & 4.77317 & -1.26015\end{array}$

$\begin{array}{lllll}\text { N64 } & -3.20219 & 0.62789 & -0.36148\end{array}$

$\begin{array}{lllll}\text { C65 } & -4.65166 & 0.36597 & -0.23819\end{array}$

$\begin{array}{llll}\text { C66 } & -2.73265 & 1.95153 & 0.10342\end{array}$ 


$\begin{array}{lccl}\text { H67 } & -5.18591 & 1.31284 & -0.06058 \\ \text { C68 } & -5.16817 & -0.22983 & -1.54304 \\ \text { C69 } & -4.9244 & -0.57117 & 0.93487 \\ \text { H70 } & -1.63863 & 1.9072 & -0.01408 \\ \text { C71 } & -3.24663 & 3.10125 & -0.76348 \\ \text { C72 } & -3.04517 & 2.16869 & 1.57945 \\ \text { H73 } & -6.23322 & -0.48 & -1.46246 \\ \text { H74 } & -5.03806 & 0.47582 & -2.3748 \\ \text { H75 } & -4.61697 & -1.1515 & -1.78208 \\ \text { H76 } & -4.48983 & -1.56041 & 0.72594 \\ \text { H77 } & -4.47479 & -0.19183 & 1.8601 \\ \text { H78 } & -6.00467 & -0.69472 & 1.08981 \\ \text { H79 } & -4.33997 & 3.19147 & -0.70537 \\ \text { H80 } & -2.81374 & 4.05411 & -0.43075 \\ \text { H81 } & -2.96346 & 2.94614 & -1.81377 \\ \text { H82 } & -4.1276 & 2.2488 & 1.75056 \\ \text { H83 } & -2.65353 & 1.34393 & 2.18936 \\ \text { H84 } & -2.58461 & 3.10603 & 1.91806 \\ \text { LI85 } & -1.71637 & -0.64281 & 0.311 \\ \text { O86 } & -1.85238 & -1.07749 & 2.23472 \\ \text { C87 } & -0.53358 & -0.90209 & 2.7948 \\ \text { C88 } & -0.0611 & -2.30802 & 3.1277 \\ \text { C89 } & -1.3629 & -2.96874 & 3.58825 \\ \text { C90 } & -2.39384 & -2.34926 & 2.64435 \\ \text { H91 } & 0.07569 & -0.41173 & 2.02483 \\ \text { H92 } & -0.61323 & -0.27697 & 3.6981 \\ \text { H93 } & 0.31967 & -2.79228 & 2.21746 \\ \text { H94 } & 0.72291 & -2.31835 & 3.89306 \\ \text { H95 } & -1.34752 & -4.06132 & 3.51716 \\ \text { H96 } & -1.58037 & -2.6915 & 4.62765 \\ \text { H97 } & -2.54089 & -2.96733 & 1.74686 \\ \text { H98 } & -3.36773 & -2.18027 & 3.11971 \\ & & & \end{array}$

Thermodynamics:

$\begin{array}{lc}\text { Zero-point correction }= & 0.658142(\text { Hartree/Particle) } \\ \text { Thermal correction to Energy= } & 0.691755 \\ \text { Thermal correction to Enthalpy= } & 0.692699 \\ \text { Thermal correction to Gibbs Free Energy= } & 0.592330 \\ \text { Sum of electronic and zero-point Energies }= & -1139.086394 \\ \text { Sum of electronic and thermal Energies }= & -1139.052782 \\ \text { Sum of electronic and thermal Enthalpies }= & -1139.051838 \\ \text { Sum of electronic and thermal Free Energies }= & -1139.152207\end{array}$




\section{Structure $23_{\mathrm{TS}}$ - Pyrrolidine portion frequency sweep only}

Input:

\#m062x/jul-cc-pVDZ freq Pop=Hirshfeld scrf=(pcm,solvent=THF)

Input Structure:

Symbolic Z-matrix:

Charge $=0$ Multiplicity $=1$

$\mathrm{N} 1 \quad 0.01912-1.19106-1.97681$

C3 $\quad 0.33312-2.66886-1.82401$

$\begin{array}{lllll}\mathrm{C} 4 & -1.17984 & -0.87684 & -2.58083\end{array}$

$\begin{array}{llll}\text { C5 } & 1.09529 & -0.31606 & -2.53499\end{array}$

$\begin{array}{llll}\text { C6 } & -1.07919 & 0.54976 & -3.03689\end{array}$

$\begin{array}{llll}\text { C7 } & 0.36299 & 0.56021 & -3.55809\end{array}$

H9 $\quad-2.07844-1.26985-2.10345$

$\begin{array}{lllll}\mathrm{H} 10 & -1.15415 & 1.28421 & -2.20212\end{array}$

H11 $\quad-1.82603 \quad 0.82412-3.79589$

H12 $\quad 0.7756 \quad 1.57942 \quad-3.6218$

$\begin{array}{lllll}\mathrm{H} 13 & 0.36451 & 0.13132 & -4.58014\end{array}$

H14 $\quad 1.83725 \quad-0.97106-3.00791$

$\begin{array}{llll}\text { C15 } & -0.75439 & -3.36877 & -1.00892\end{array}$

$\begin{array}{lllll}\mathrm{C} 16 & 0.39372 & -3.30837 & -3.21704\end{array}$

$\begin{array}{llll}\mathrm{C} 17 & 1.67251 & -2.85945 & -1.1161\end{array}$

H18 $\quad-1.7504 \quad-3.20476-1.43715$

H19 $\quad-0.56003 \quad-4.44982-1.03045$

$\begin{array}{lllll}\mathrm{H} 20 & -0.72948 & -3.01701 & 0.02658\end{array}$

$\mathrm{H} 21 \quad-0.60563 \quad-3.37523 \quad-3.65711$

$\begin{array}{lllll}\mathrm{H} 22 & 1.03427 & -2.72886 & -3.89147\end{array}$

$\mathrm{H} 23 \quad 0.81357 \quad-4.31944-3.12187$

H24 $\quad 2.51171 \quad-2.52628-1.73737$

H25 $\quad \begin{array}{lllll} & 1.66186 & -2.29734 & -0.18042\end{array}$

H26 $\quad 1.80873 \quad-3.9305 \quad-0.91041$

Thermodynamics:

Zero-point correction $=$

Thermal correction to Energy=

0.214528 (Hartree/Particle)

Thermal correction to Enthalpy=

0.223861

0.224805

Thermal correction to Gibbs Free Energy $=\quad 0.181340$

Sum of electronic and zero-point Energies= $-368.170257$

Sum of electronic and thermal Energies $=$ $-368.160925$

Sum of electronic and thermal Enthalpies= $-368.159981$

Sum of electronic and thermal Free Energies $=\quad-368.203445$ 